\title{
Targeting PI3K in cancer: mechanisms and advances in clinical trials
}

\author{
Jing Yang, Ji Nie, Xuelei Ma, Yuquan Wei, Yong Peng and Xiawei Wei
}

\begin{abstract}
Phosphatidylinositol-3-kinase (PI3K)/AKT/mammalian target of rapamycin (mTOR) signaling is one of the most important intracellular pathways, which can be considered as a master regulator for cancer. Enormous efforts have been dedicated to the development of drugs targeting PI3K signaling, many of which are currently employed in clinical trials evaluation, and it is becoming increasingly clear that PI3K inhibitors are effective in inhibiting tumor progression. PI3K inhibitors are subdivided into dual PI3K/mTOR inhibitors, pan-PI3K inhibitors and isoform-specific inhibitors. In this review, we performed a critical review to summarize the role of the PI3K pathway in tumor development, recent PI3K inhibitors development based on clinical trials, and the mechanisms of resistance to PI3K inhibition.
\end{abstract}

Keywords: PI3K, mTOR, Cancer, Target therapy

\section{Highlights}

Activation of the PI3K pathway contributes to the development of tumor PI3K is an attractive therapeutic direction in the treatment of cancer. Inhibition of PI3K signaling is effective in the treatment of several types of cancer. Intrinsic and acquired resistance limits the therapeutic efficacy of PI3K inhibitors.

\section{Introduction}

Phosphatidylinositol-3-kinase (PI3K)/AKT/mammalian target of rapamycin (mTOR) signaling is one of the most important intracellular pathways, which regulates cell growth, motility, survival, metabolism, and angiogenesis $[1,2]$. Activation of the PI3K/AKT/mTOR pathway contributes to the development of tumor and resistance to anticancer therapies [3]. MicroRNA (miRNA) and long non-coding RNA (lncRNA), the two most studied classes of non-coding RNA (ncRNA), are crucial regulators of gene expression [4]. These two types of ncRNA and $\mathrm{PI} 3 \mathrm{~K} / \mathrm{AKT} / \mathrm{mTOR}$ pathway are in tight conjunction during oncogenesis $[5,6]$. The PI3K/AKT/mTOR pathway has been found to be dysregulated almost in all human cancers, such as breast cancer, colorectal cancer, and hematologic malignancies, which emphasizes the value

\footnotetext{
* Correspondence: xiaweiwei@scu.edu.cn

Laboratory of Aging Research and Cancer Drug Target, State Key Laboratory of Biotherapy and Cancer Center, National Clinical Research Center for Geriatrics, West China Hospital, Sichuan University, Chengdu 610041, Sichuan, China
}

of targeting this pathway as a potential therapeutic direction in the treatment of cancer [7]. Inhibition of PI3K can result in both decreased cellular proliferation and increased cellular death [8]. Small molecule inhibitors of PI3K include PI3K/mTOR inhibitors, pan-PI3K inhibitors, and isoform-selective PI3K inhibitors. The safety and efficacy of these therapeutic approaches have been investigated in a wide range of preclinical and clinical trials, and it is becoming increasingly clear that PI3K inhibitors are effective in inhibiting tumor progression. For example, PI3K delta-specific inhibitor idelalisib is the first PI3Ki compound approved by United States Food and Drug Administration (FDA) and is proved to be effective in the cancer treatment [9]. In this review, we summarized the role of the PI3K signaling in tumor progression, recent PI3K inhibitors development based on clinical trials, and the mechanisms of resistance to PI3K inhibition.

\section{PI3K signal pathway}

\section{Signal transduction pathways}

PI3K is a group of plasma membrane-associated lipid kinases, consisting of three subunits: p85 regulatory subunit, p55 regulatory subunit, and p110 catalytic subunit [10]. According to their different structures and specific substrates, PI3K is divided into 3 classes: classes I, II, and III $[1,2]$. Class I PI3Ks comprised of class IA and class IB PI3Ks. Class IA PI3K, a heterodimer of p58 
regulatory subunit and p110 catalytic subunit, is the type most clearly implicated in human cancer [11]. Class IA PI3K contains $\mathrm{p} 110 \alpha, \mathrm{p} 110 \beta$ and $\mathrm{p} 110 \delta$ catalytic subunits produced from different genes (PIK3CA, PIK3CB and PIK3CD, respectively), while $\mathrm{p} 110 \gamma$ produced by PIK3CG represents the only catalytic subunit in class IB PI3K [12]. The p85 regulatory subunit is composed of $\mathrm{p} 85 \mathrm{a}$ (p85a, p55a and p50a splice variants), p85b and p55g, which are encoded by the genes PIK3R1, PIK3R2 and PIK3R3, respectively [2]. As an integration point for p110 activation and downstream molecular, p85 regulatory subunit binds and integrates signals from various transmembrane and intracellular proteins, including tyrosine kinase-linked receptors, protein kinase C (PKC), Src homology 2 domain-containing protein tyrosine phosphatase 1 (SHP1), Rac, Rho, hormonal receptors, Src, as well as mutated Ras [8]. The overview of PI3K/ AKT/mTOR signaling pathway was shown in Fig. 1.

\section{Activation of PI3K signaling}

Under baseline conditions, the p110 catalytic subunit is stabilized by dimerization with regulatory p 85 subunit. In physiologic conditions, PI3K is normally activated by a variety of extracellular stimuli, such as growth factors, cytokines, and hormones [13]. Upon activation, PI3K catalyzes the phosphorylation of PtdIns(4,5) P2(PIP2) to produce PtdIns(3,4,5) P3(PIP3), a second messenger that binds and recruits a subset of pleckstrin-homology $(\mathrm{PH})$, FYVE, Phox (PX), C1, C2 or other lipid-binding domains of downstream targets to the cell membrane. A variety of signaling proteins, such as kinases AKT and PDK1 can bind to the lipid products of PI3K and thereby localize to the cell membrane to activate cell growth and cell survival pathways [14]. Phosphatase and tensin homologue deleted on chromosome 10 (PTEN) regulates the pathway by dephosphorylating PIP3 to PIP2 and thus prevents activation of downstream kinases [8].

In the last decade or so, ncRNAs have emerged as important regulators of a wide range of genes and PI3K/ AKT/mTOR pathway $[5,6]$. ncRNAs function as both upstream mediators and downstream effectors to affect PI3K pathway activities. Of importance, ncRNAs have been reported to directly or indirectly target multiple key components (PI3K, AKT, mTOR and PTEN) in the PI3K pathway, regulating the activity of PI3K signaling. However, the exact mechanisms through which lncRNAs

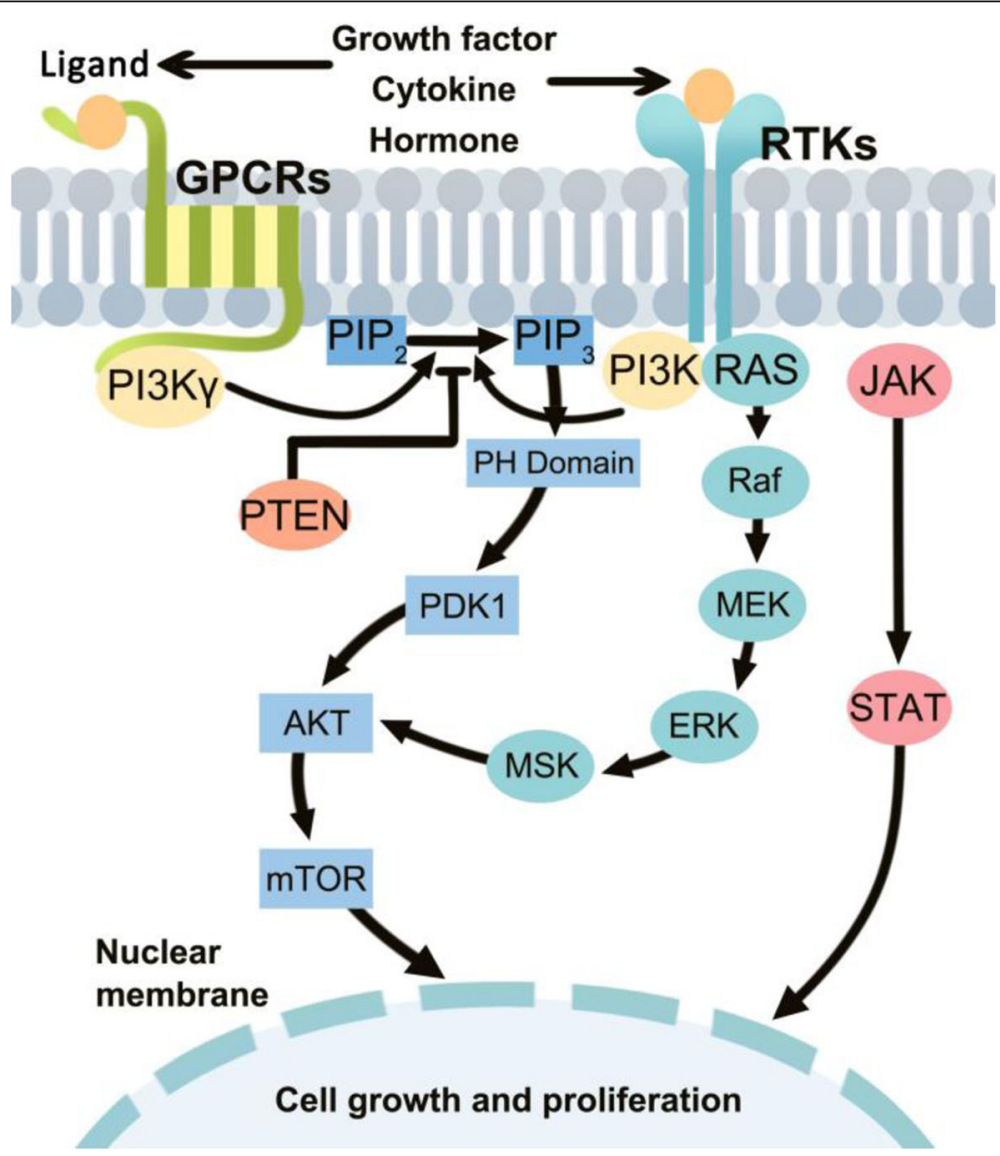

Fig. 1 The overview of PI3K/AKT/mTOR signaling pathway 
directly or indirectly affect PI3K have not been fully studied.

\section{PI3K signaling in human cancer}

Over the past decades, PI3K signaling pathway is believed to be deregulated in a wide spectrum of human cancers. Mutations of the kinases and/or decreased expression of PTEN lead to neoplastic transformation, underscoring its central role in human carcinogenesis $[8$, 15]. PI3K pathway is deregulated through a variety of mechanisms, including loss or inactivation of the tumor suppressor PTEN, mutation or amplification of PI3K, as well as activation of tyrosine kinase growth factor receptors or oncogenes upstream of PI3K [16-18].

\section{Loss or inactivation of PTEN}

PTEN, a negative regulator of PI3K pathway, acts as a direct antagonist of PI3K action through dephosphorylation of PIP3. Dimeric PTEN complexes have higher activity than PTEN monomers in PIP3 dephosphorylation and PI3K signaling regulation $[19,20]$. PTEN is a well characterized tumor suppressor with growth, survival and metabolic regulatory functions, and its loss or inactivation of function is frequently observed in both heritable and sporadic malignances, including brain cancer, breast cancer, and prostate cancer [21-23]. Furthermore, it has been shown that small changes in PTEN expression contribute to major consequences for normal cellular function [24]. In PTEN knock-in mice harboring two cancer-associated PTEN mutations, PTENC124S and PTENG129E inhibit the PTEN lipid-phosphatase activity in a dominant negative manner, leading to increased activity of PI3K signaling and tumorigenesis [20]. Moreover, in PTEN-deficient cancer, the main carcinogenic driving force is the overactivation of AKT caused by the loss of PTEN lipid phosphatase function [20, 25].

\section{Mutation or amplification of PI3K}

PIK3CA (phosphatidylinositol 3-kinase, catalytic, $\alpha$-polypeptide), the gene encoding the p110 $\alpha$ subunit, are frequently mutated or amplified in the most common human cancers, such as breast cancers, colon cancer, gastric cancer, cervical cancer, prostate cancer, and lung cancer [26-31]. Most mutations cluster around two hotspots: E545K (exon 9) in the helical phosphatidylinositol kinase homology domain, which reduces inhibition of $\mathrm{p} 110 \alpha$ by the regulatory subunit $\mathrm{p} 85$; H1047 (exon 20) near the end of the catalytic domain, which increases interaction of $\mathrm{p} 110 \alpha$ with lipid membranes [32, 33]. E542K is also one of the most frequently observed PIK3CA mutations [33, 34]. In colorectal cancer, exon 9 plays a more important role than exon 20 , whereas in endometrial cancer, the opposite pattern was described, suggesting that different mutations of
PIK3CA may have specific effects on downstream carcinogenic signals [35]. It is worth noting that the coexistence of mutations in helical domain and kinase domain leads to synergistic enhancement of p110 activity and enhancement of the tumorigenicity effects [35]. In addition to the two hotspot mutations, mutations on C2 domain are also important components of PIK3CA mutations [36]. Such deregulation of PI3K pathway promotes cell proliferation and migration, glucose transport and catabolism, cytoskeletal rearrangements, and angiogenesis, playing an important role in tumor initiation, progression, and maintenance [27]. In addition, the tumorigenic potential of these mutations was confirmed in experimental research using genetically engineered mouse models (GEMMs) [37-39].

In contrast, mutations in the other catalytic subunits $\mathrm{p} 110 \beta, \mathrm{p} 110 \gamma$ and $\mathrm{p} 110 \delta$ are rare, and overexpression of these wild-type catalytic subunits is sufficient to induce an oncogenic phenotype in cultured cells [34, 40]. Subunit p110 $\beta$ plays an important role in stimulating cell proliferation, invasiveness, as well as tumorigenesis in prostate and breast cancer [41-43]. The precise mechanisms of p110 $\beta$ activation in cancer are still not well established. However, it has been reported that it can occur through G protein-coupled receptor (GPCRs) [44]. E633K, a p110 $\beta$ helical domain mutation, was first reported in a HER2-positive breast cancer patient [45]. E633K might enhance p110 3 's basal association with membranes and thus activates $\mathrm{p} 110 \beta$ [43]. The $\mathrm{p} 110 \beta$ has been suggested to be responsible for the reaccumulation of PIP3 and reactivation of AKT in HER2-amplified cancers treated with a p110 $\alpha$-specific inhibitor, and concomitant inhibition of $\mathrm{p} 110 \alpha$ and $\mathrm{p} 110 \beta$ induces greater antitumor efficacy in HER2-amplified and PIK3CA mutant breast cancers. In endometrial cancer, occurrence of PIK3CB mutations (D1067V and A1048V within the kinase domain) has been reported [46, 47]. PI3K $\delta$ is primarily expressed in the cells of hematopoietic lineage and is activated by cytokine receptors, antigen receptors, growth factor receptors and costimulatory receptors $[48,49]$. PI3K $\delta$ is important in T and B cells development and activation. PI3K $\delta$ blockade increases genomic instability by an activation-induced cytidine deaminase (AID)-dependent mechanism in B cells [50]. Gain-of-function (GOF) mutations in PI3K $\delta$ result in a range of developmental and functional deficiencies of $\mathrm{B}$ and $\mathrm{T}$ cell that compromise host defense. Loss-of-function (LOF) mutations lead to much more severe B cell lymphopenia and agammaglobulinemia, but not $\mathrm{T}$ cell senescence [51]. In acute myeloid leukemia (AML), PI3K $\delta$ is critical in activation of AKT and cell proliferation [52]. Point mutations of $\mathrm{p} 110 \delta$ have been described in a panel of diffuse large B-cell lymphomas [53]. Moreover, p110 $\delta$ protein has been detected in cells of melanocytic or breast origin and it has been reported to regulate cell migration in breast 
cancer lines and tumor progression [54]. PI3K $\gamma$ is abundantly expressed in immune cells of myeloid origin but not cancer cells, which regulates innate immunity in both inflammation and cancer [55]. P110 $\gamma$ contributes to chemotactic responses, as well as reactive oxygen species production in neutrophils [56]. PI3KY may possibly be able to promote solid tumor neovascularization indirectly by regulating the immune-suppressive TAM subset, which is a major source of VEGF $\alpha$ [57].

\section{Non-coding RNA and other factors in regulation of PI3K pathway}

In addition to inherent aberrations in members of the PI3K pathway, pathologic signaling through this pathway can also occur in other ways, including tyrosine kinase growth factor receptors (e.g. human epidermal growth factor receptor 2 and insulin-like growth factor -1 receptor), cell adhesion molecules (e.g. integrins, GPCR), and oncogenes (e.g. RAS) $[1,58,59]$. The interactions between ncRNAs and PI3K signaling in cancer have been studied. For example, the lncRNA CRNDE which promote cell proliferation through activating PI3K signaling, is highly expressed in patients with non-small cell lung cancer, colorectal cancer, gastric cancer, cervical cancer, heptaocellular carcinoma and gallbladder cancer [60-65]. In addition to activating PI3K pathway, some ncRNAs have been reported to inhibit the activity of PI3K signaling. lncRNA GAS5 expression is lower in tumor cells compared to normal cells; its over-expression inhibits tumor cell proliferation and migration while treatment with PI3K activator reduces the inhibitory effects [66-72]. Table 1 shows the examples of IncRNA that interact with PI3K signaling in different types of cancer.

\section{PI3K inhibitors}

PI3K are believed to be one of the key therapeutic targets for cancer treatment based on the observation that hyperactivity of PI3K signaling is significantly correlated with human tumor progression, increased tumor microvessel density and enhanced chemotaxis and invasive potential of cancer cells. Enormous efforts have been dedicated to the development of drugs targeting PI3K signaling, many of which are currently employed in clinical trials evaluation. Important ongoing clinical trials with PI3K-targeted therapies were summarized in Table 2. PI3K inhibitors are subdivided into dual PI3K/mTOR inhibitors, pan-PI3K inhibitors and isoform-specific inhibitors. The drugs targeting PI3K in clinical trial were shown in Table 3 and Fig. 2.

\section{Dual PI3K/mTOR inhibitors NVP-BEZ235 (Dactolisib)}

NVP BEZ235 (dactolisib) is a dual PI3K/mTOR inhibitor and is currently in Phase I/II clinical trials. It is an imidazo [4,5-c] quinoline derivative compound that binds to the ATP-binding cleft of PI3K and mTOR kinase, inhibiting their catalytic activities [25]. BEZ235 exhibited satisfactory anticancer effects in preclinical studies in several types of cancer, including the following: triple-negative breast cancer, lung cancer, melanoma, colorectal cancer, renal cancer, prostate cancer, lymphoma, and mucinous adenocarcinoma of the ovary [73-85]. However, the clinical trials of BEZ235 were not satisfactory. A phase I study investigated maximum tolerated dose (MTD), recommended dose for expansion (RDE), safety and antitumor activity of BEZ235, in combination with abiraterone acetate [86]. In this study, dose escalation was stopped after $200 \mathrm{mg}$ bid due to challenging safety and tolerability profile; the most common adverse events (AEs) were diarrhea (78\%), nausea (61\%) and stomatitis (39\%). Moreover, no objective response and few prostate specific antigen (PSA) decreases were reported. Limited efficacy and poor tolerance of BEZ235 combined with everolimus (BEZ235: 200, 400, or $800 \mathrm{mg}$ daily; everolimus: $2.5 \mathrm{mg}$ daily; 28 -day cycles) in patients with advanced solid malignancies were reported in a phase Ib trial [87].

In a Phase II Study, BEZ235 was poorly tolerated by patients with everolimus-resistant pancreatic neuroendocrine tumor at 400 or $300 \mathrm{mg}$ bid doses, and the estimated 16-week progression-free survival (PFS) rate was 51.6\% [88]. Treatment-related grade 3/4 AEs including hyperglycaemia, nausea, diarrhoea, and vomiting occurred in $72.7 \%$ patients at $400 \mathrm{mg}$ and $40.0 \%$ patients at $300 \mathrm{mg}$; $95.0 \%$ of the patients in the $300 \mathrm{mg}$ group and all patients in the $400 \mathrm{mg}$ group experienced at least one $\mathrm{AE}$ relating to the treatment [88]. Treatment with BEZ235 in mTOR inhibitor-naive patients with advanced pancreatic neuroendocrine tumors demonstrated poorer efficacy and tolerability compared with everolimus in another Phase II study [89]. Phase I studies of BEZ235 in patients with advanced breast cancer and advanced renal cancer, reported that BEZ235 was not enough to achieve a satisfactory antitumor effect with a favorable safety profile. Currently, several clinical studies of BEZ235 among patients with relapsed or refractory acute leukemia and patients with metastatic breast cancer are ongoing.

\section{GDC-0980 (Apitolisib, RG7422)}

GDC-0980 (apitolisib, RG7422) is a potent, orally bioavailable inhibitor of class I PI3K and mTOR kinase (TORC1/ 2). Several preclinical studies have assessed this agent's activity in a variety of solid tumors. A phase I trial assessed the safety, tolerability, and preliminary antitumor effects of GDC-0980 in patients with solid tumors [90]. In this study, $2-70 \mathrm{mg}$ daily GDC-0980 was administered to patients for days $1-21$ or $1-28$ of 28 -day cycles. The main AEs from this agent were hyperglycemia, rash, liver dysfunction and diarrhea. This phase I study concluded that GDC-0980 has a narrow therapeutic window, and dose of $40 \mathrm{mg}$ 28/28 days was reasonably tolerated. More recently, 
Table 1 Important long non-coding RNA that interact with PI3K signaling in different cancer

\begin{tabular}{|c|c|c|c|c|}
\hline LncRNAs & $\begin{array}{l}\text { Up- or down- } \\
\text { regulation }\end{array}$ & Cancer type & Affected biological process (Involved factors) & References \\
\hline CRNDE & Up & $\begin{array}{l}\text { Gallbladder carcinoma; Non-small cell lung } \\
\text { carcinoma; Colorectal cancer; Gastric cancer; } \\
\text { Cervical cancer; Heptaocellular cancer }\end{array}$ & $\begin{array}{l}\text { Cell roliferation, growth, migration, invasion } \\
\text { and apoptosis; glucose and lipid metabolism } \\
\text { (Gene expression in PI3K pathway: MMP-9, } \\
\text { JUK-1, ERK and AKT) }\end{array}$ & [60-65] \\
\hline OIP5-AS1 & Up & Multiple myeloma; Osteosarcoma & $\begin{array}{l}\text { Cell proliferation, cycle and apoptosis; } \\
\text { cisplatin resistance, } \\
\text { (miRNA-410, miRNA-340-5p and expression } \\
\text { of lysophosphatidic acid acyltransferase) }\end{array}$ & {$[201,213]$} \\
\hline CCAT1 & Up & Thyroid carcinoma; Squamous cell carcinomas & $\begin{array}{l}\text { Cell proliferation, migration, and invasion } \\
\text { (miRNA-143; EGFR expression) }\end{array}$ & {$[214,215]$} \\
\hline H19 & Up & Retinoblastoma, Melanoma & $\begin{array}{l}\text { Cell viability, migration, invasion, and } \\
\text { apoptosis } \\
\text { (miRNA-143, RUNX2, Phosphorylation } \\
\text { of key kinases) }\end{array}$ & {$[216,217]$} \\
\hline HOTAIR & UP & $\begin{array}{l}\text { Gastric cancer; Adenocarcinoma of } \\
\text { esophagogastric junction; Leukemia; } \\
\text { Melanoma; Gliomas }\end{array}$ & $\begin{array}{l}\text { Cell proliferation, metastasis and apoptosis; } \\
\text { cisplatin resistance; acquired multidrug } \\
\text { resistance to imatinib } \\
\text { (miRNA-143, miRNA-34a, miRNA-152-3p, } \\
\text { miRNA-126, miRNA-326, FGF1) }\end{array}$ & $\begin{array}{l}{[202,203,218-} \\
222]\end{array}$ \\
\hline NEAT1 & Up & Myeloma; Cervical carcinoma; Gastric cancer & $\begin{array}{l}\text { Cell proliferation, viability, migration, } \\
\text { invasion, apoptosis, and cell cycle. } \\
\text { (microRNA-17) }\end{array}$ & {$[223-226]$} \\
\hline HULC & Up & $\begin{array}{l}\text { Bladder cancer; Leukemia; Gliomas; } \\
\text { Osteosarcoma; Liver cancer; Gastric cancer }\end{array}$ & $\begin{array}{l}\text { Cell viability, growth, migration, invasion } \\
\text { and autophagy } \\
\text { (PTEN, miRNA15a, autophagy-P62, miRNA-122) }\end{array}$ & {$[227-232]$} \\
\hline AB073614 & Up & Colorectal cancer & Cell cycle, proliferation, migration, and invasion & [233] \\
\hline PTTG3P & Up & Hepatocellular carcinoma & $\begin{array}{l}\text { Cell proliferation, migration, invasion, } \\
\text { tumorigenesis and metastasis }\end{array}$ & [234] \\
\hline MALAT1 & Up & $\begin{array}{l}\text { Cervical cancer; Epithelial ovarian cancer; } \\
\text { Breast cancer; Osteosarcoma; Cholangiocarcinoma; } \\
\text { Non-small cell lung carcinoma; Hepatocellular } \\
\text { carcinoma; Renal cell carcinoma }\end{array}$ & $\begin{array}{l}\text { Cell proliferation, invasion, metastasis, viability } \\
\text { and mobility; stemness-related factor activation; } \\
\text { epithelial-to-mesenchymal transition; cisplatin } \\
\text { resistance } \\
\text { (PI3Kp85a, miRNA-22-3p, miRNA195, } \\
\text { miRNA-124, MiRNA-101-3p, miRNA-129-5p) }\end{array}$ & {$[235-245]$} \\
\hline ATB & Up & Bladder cancer; Prostate carcinoma & $\begin{array}{l}\text { Cell proliferation, migration and invasion; } \\
\text { mitogenic; epithelial-mesenchymal transition } \\
\text { (microRNA-126, KRAS,) }\end{array}$ & {$[246,247]$} \\
\hline BC087858 & Up & Non-small-cell lung cancer & $\begin{array}{l}\text { Cells invasion; resistance to EGFR-TKIs } \\
\text { (ZEB1, Snail.) }\end{array}$ & [248] \\
\hline Linc00659 & Up & Colorectal cancer & Cell growth inhibition and apoptosis & [249] \\
\hline Linc00152 & Up & $\begin{array}{l}\text { Lung cancer; } \\
\text { Gallbladder cancer }\end{array}$ & $\begin{array}{l}\text { Cell proliferation, invasion, migration, } \\
\text { apoptosis, and G1 phase rates }\end{array}$ & {$[250,251]$} \\
\hline Linc00462 & Up & Hepatocellular carcinoma & Cell proliferation, invasion and migration & [252] \\
\hline Linc01296 & Up & Prostate cancer; Colorectal cancer & $\begin{array}{l}\text { Tumorigensis, cell proliferation, migration, } \\
\text { invasion, and liver metastasis; } \\
\text { epithelial-mesenchymal transition; } \\
\text { chemoresistance to 5-fluorouracil } \\
\text { (miRNA-26a, mucin1, GALNT3) }\end{array}$ & {$[253,254]$} \\
\hline Linc003121 & Down & Thyroid cancer & Cell proliferation, Invasion, and tumorigenicity & [255] \\
\hline UCA1 & Up & Gastric cancer; Bladder cancer & $\begin{array}{l}\text { Cell proliferation, migration, invasion, } \\
\text { apoptosis and cell cycle }\end{array}$ & [256-259] \\
\hline ecCEBPA & Up & Gastric cancer; Hepatic cancer & Disease progression & {$[257,260]$} \\
\hline Ftx & Up & Hepatocellular carcinoma & $\begin{array}{l}\text { Cell growth } \\
\text { (miRNA-545, RIG-I) }\end{array}$ & [261] \\
\hline RMEL3 & Up & Melanoma & $\begin{array}{l}\text { Cell survival and proliferation } \\
\text { (PTEN) }\end{array}$ & [262] \\
\hline
\end{tabular}


Table 1 Important long non-coding RNA that interact with PI3K signaling in different cancer (Continued)

\begin{tabular}{|c|c|c|c|c|}
\hline LncRNAs & $\begin{array}{l}\text { Up- or down- } \\
\text { regulation }\end{array}$ & Cancer type & Affected biological process (Involved factors) & References \\
\hline LncARSR & Up & Hepatocellular Carcinoma & $\begin{array}{l}\text { Doxorubicin resistance } \\
\text { (PTEN) }\end{array}$ & {$[206]$} \\
\hline BDLNR & Up & Cervical cancer & $\begin{array}{l}\text { Cell proliferation, migration, and death; } \\
\text { anti-cancer effects of baicalein } \\
\text { (YBX1, PIK3CA promoter) }\end{array}$ & [263] \\
\hline ANRIL & Up & $\begin{array}{l}\text { Cervical cancer; Osteosarcoma; } \\
\text { Gliomas }\end{array}$ & $\begin{array}{l}\text { Cell proliferation, migration, invasion, } \\
\text { and apoptosis } \\
\text { (miRNA-34a, Sirt1) }\end{array}$ & {$[264-266]$} \\
\hline ROR & Up & Non-small-cell lung cancer & $\begin{array}{l}\text { Cell proliferation, migration, and invasion; } \\
\text { cisplatin resistance }\end{array}$ & [267] \\
\hline PlncRNA-1 & Up & Colorectal cancer & $\begin{array}{l}\text { Cell proliferation, migration, invasion, } \\
\text { and apoptosis }\end{array}$ & [268] \\
\hline MYD88 & Up & Hepatocellular carcinoma; & $\begin{array}{l}\text { Cell proliferation and metastasis } \\
\text { (H3K27AC) }\end{array}$ & [269] \\
\hline RP4 & Down & Colorectal cancer & $\begin{array}{l}\text { Cell proliferation, growth, and early apoptosis } \\
\text { (SH3GLB1, miRNA-7-5p) }\end{array}$ & {$[270]$} \\
\hline OIP5-AS1 & Down & Osteosarcoma, myeloma & $\begin{array}{l}\text { Cell growth; cisplatin resistance } \\
\text { (miRNA-340-5p, LPAATbeta, miRNA-410, KLF10) }\end{array}$ & {$[201,213]$} \\
\hline MEG3 & Down & $\begin{array}{l}\text { Endometrial carcinoma; Breast cancer; } \\
\text { Cervical cancer; Pancreatic cancer; } \\
\text { Lymphoma; Gliomas }\end{array}$ & $\begin{array}{l}\text { Cell proliferation, migration, invasion, metastasis, } \\
\text { and apoptosis; autophagy; glycolysis; } \\
\text { epithelial-mesenchymal transition; chemoresistance } \\
\text { (Combine directly with PI3K, miRNA-21, } \\
\text { cytomembrane translocation of AKT) }\end{array}$ & {$[271-277]$} \\
\hline GAS5 & Down & $\begin{array}{l}\text { Colorectal cancer; Esophageal squamous } \\
\text { cell carcinoma; Breast cancer; Malignant } \\
\text { pleural mesothelioma; Osteosarcoma; } \\
\text { Prostate cancer }\end{array}$ & $\begin{array}{l}\text { Cell proliferation and migration, viability, migration } \\
\text { and invasion; apoptotic responses to conventional } \\
\text { chemotherapies } \\
\text { (miRNA-203a, TIMP2, miRNA-196a-5p, FOXO1) }\end{array}$ & {$[66-72]$} \\
\hline RNA-422 & Up & Colorectal cancer & Cell proliferation, migration, and invasion & [278] \\
\hline
\end{tabular}

a single arm, open-label trial phase II study in recurrent or persistent endometrial carcinoma patients reported that anti-tumor activity of $40 \mathrm{mg}$ GDC-0980 daily was limited by tolerability, especially in diabetic patients, and patients with mutations of PI3K pathway may benefit more from GDC-0980 [91].

In another phase II study, 85 patients with metastatic renal cell carcinoma were randomly assigned to apitolisib $40 \mathrm{mg}$ QD or to everolimus $10 \mathrm{mg}$ QD. Patients receiving GDC-0980 were shown to have poorer median PFS (3.7 vs6.1 months; hazard ratio (HR) $2.12 ; p<0.01$ ) than patients receiving everolimus, while meidan overall survival (OS) was not significantly different but trended in favor of patients receiving everolimus (16.5 v 22.8 months; HR $1.77 ; p=0.06)$ [92, 93]. However, GDC-0980 was reported to be well tolerated and to have early signs of anti-tumor activity in patients with advanced solid tumors or non-Hodgkin lymphoma, with an $80 \%$ decrease in measurable tumor marker [90]. A PhaseI/II study of GDC-0980 in patients with prostate cancer is ongoing.

In a phase Ib study of GDC-0980 in combination with capecitabine, 19 patients with advanced solid tumors and colorectal cancer were enrolled [94]. Confirmed partial responses (PR) were observed in one head and neck squamous cell cancer patient and one colorectal cancer patient with PIK3CA and KRAS mutations, which indicated preliminary anti-tumor activity of GDC-0980 in combination with capecitabine. GDC-0980 combined with fluoropyrimidine-based regimens was also demonstrated to be well tolerated, with confirmed antitumor activity [95].

\section{PF-04691502 and PF-05212384 (Gedatolisib, PKI-587)}

PF-04691502 and PF-05212384 (gedatolisib, PKI-587) are potent ATP competitive dual class-I PI3K/ mTOR kinases inhibitors. Preclinical studies demonstrated that PI3K-mTOR inhibition with PF-04691502 can enhance TP53/p73 expression and significantly inhibit tumor growth in head and neck squamous cell carcinomas [96]. In cancer cell lines with PI3Ka mutation and PTEN deletion, PF-04691502 can reduce phosphorylation of AKT and S6RP, thus inhibit cell proliferation [97]. PF-05212384 were reported to suppress a negative feedback loop mediated by mTORC2, leading to MEK/ERK over-activation in pancreatic cancer cells [98]. PF-05212384 causes strong attenuation of cell cycle and G0/G1 arrest, as well as induction of apoptosis in neuroendocrine tumor cells [99]. Phase I study of PF-04691502 in 23 patients with advanced solid tumors recommended that $8 \mathrm{mg}$ orally once 
Table 2 Important ongoing clinical trials with PI3K-targeted therapies

\begin{tabular}{|c|c|c|c|c|c|}
\hline Conditions & $\begin{array}{l}\text { Sample } \\
\text { size }\end{array}$ & Design & Phase & Status & Trial number \\
\hline \multicolumn{6}{|c|}{ NVP-BEZ235 (BEZ235, Dactolisib) Dual PI3K/mTOR inhibitor } \\
\hline $\begin{array}{l}\text { Acute Lymphoblastic Leukemia; Acute Chronic } \\
\text { Myelogenous Leukemia With Crisis of Blast Cells }\end{array}$ & 23 & BEZ235 & । & $\begin{array}{l}\text { Active not } \\
\text { recruiting }\end{array}$ & NCT01756118 \\
\hline \multicolumn{6}{|l|}{ GDC-0084 (RG7666) Dual PI3K/mTOR inhibitor } \\
\hline Glioblastoma, Adult & 66 & GDC-0084 & $\|$ & Recruiting & NCT03522298 \\
\hline Brain and Central Nervous System Tumors & 41 & Radiation therapy+ GDC-0084 & । & $\begin{array}{l}\text { Not yet } \\
\text { recruiting }\end{array}$ & NCT03696355 \\
\hline \multicolumn{6}{|l|}{$\begin{array}{l}\text { GDC-0980 (Apitolisib, RG7422) Dual PI3K/mTOR } \\
\text { inhibitor }\end{array}$} \\
\hline Prostate Cancer & 273 & Abiraterone Acetate +/- (GDC-0980//patasertib) & $|/| \mid$ & $\begin{array}{l}\text { Active not } \\
\text { recruiting }\end{array}$ & NCT01485861 \\
\hline \multicolumn{6}{|l|}{ LY3023414 Dual PI3K/mTOR inhibitor } \\
\hline $\begin{array}{l}\text { Endometrial Cancer; Recurrent } \\
\text { Endometrial Cancer }\end{array}$ & 25 & LY3023414 & $\|$ & Recruiting & NCT02549989 \\
\hline $\begin{array}{l}\text { Advanced Malignant Solid Neoplasm; Ann } \\
\text { Arbor Stage III/IV Childhood } \\
\text { Non-Hodgkin Lymphoma }\end{array}$ & - & LY3023414 & $\|$ & Recruiting & NCT03155620 \\
\hline $\begin{array}{l}\text { Metastatic Colorectal Neoplasm; Metastatic } \\
\text { Breast Cancer }\end{array}$ & 205 & $\begin{array}{l}\text { Prexasertib+Cisplatin/Cetuximab/ } \\
\text { Pemetrexed/5-FU/LY3023414 }\end{array}$ & । & Recruiting & NCT02124148 \\
\hline Advanced or Metastatic Solid Tumors & 163 & $\begin{array}{l}\text { LY3039478 + LY3023414/Taladegib/ } \\
\text { Abemaciclib/Cisplatin/Gemcitabine/Carboplatin }\end{array}$ & । & Recruiting & NCT02784795 \\
\hline NSCLC & 150 & $\begin{array}{l}\text { Abemaciclib+LY3023414/Pemetrexed/ } \\
\text { Gemcitabine/Ramucirumab/Pembrolizumab }\end{array}$ & । & $\begin{array}{l}\text { Active not } \\
\text { recruiting }\end{array}$ & NCT02079636 \\
\hline Prostate Cancer Metastatic & 144 & Enzalutamide +/- LY3023414 & ॥ & Recruiting & NCT02407054 \\
\hline $\begin{array}{l}\text { Advanced Non-Hodgkin Lymphoma; } \\
\text { Metastatic Breast Cancer; Advanced } \\
\text { Mesothelioma; Advanced NSCLC }\end{array}$ & 130 & $\begin{array}{l}\text { LY3023414 + Midazolam/Fulvestrant OR } \\
\text { LY3023414 + Pemetrexed/Cisplatin OR } \\
\text { LY3023414 +/- (Abemaciclib+ Letrozole) }\end{array}$ & । & Recruiting & NCT01655225 \\
\hline Pancreatic Ductal Adenocarcinoma & 231 & $\begin{array}{l}\text { Abemaciclib+/- LY3023414 } \\
\text { VS Gemcitabine/Capecitabine }\end{array}$ & $\|$ & $\begin{array}{l}\text { Active not } \\
\text { recruiting }\end{array}$ & NCT02981342 \\
\hline Breast Neoplasms & 198 & LY3023414 + LY2835219 + Fulvestrant & । & Recruiting & NCT02057133 \\
\hline $\begin{array}{l}\text { Advanced Malignant Solid Neoplasm; } \\
\text { Ann Arbor Stage III/ IV Non-Hodgkin Lymphoma }\end{array}$ & 144 & LY3023414 & $\|$ & Recruiting & NCT03213678 \\
\hline Endometrial Cancer & 62 & (LY3023414 + Abemaciclib) +/- Letrozole & $\|$ & $\begin{array}{l}\text { Not yet } \\
\text { recruiting }\end{array}$ & NCT03675893 \\
\hline \multicolumn{6}{|c|}{ PF-05212384 (Gedatolisib, PKI-587) Dual PI3K/mTOR inhibitor } \\
\hline $\begin{array}{l}\text { Therapy-related Acute Myeloid Leukemia } \\
\text { and Myelodysplastic Syndrome; Relapsed } \\
\text { Acute Myeloid Leukemia; de Novo Acute } \\
\text { Myeloid Leukemia at Diagnostic }\end{array}$ & 10 & PF-05212384 & $\|$ & $\begin{array}{l}\text { Active not } \\
\text { recruiting }\end{array}$ & NCT02438761 \\
\hline Neoplasm & 124 & PF-05212384 + Docetaxel/Cisplatin/Dacomitinib & । & Recruiting & NCT01920061 \\
\hline $\begin{array}{l}\text { Lung Cancer Squamous Cell; Solid Tumors; } \\
\text { Head \& Neck Cancer; Pancreatic Cancer }\end{array}$ & 96 & PF-05212384 + Palbociclib & । & Recruiting & NCT03065062 \\
\hline NSCLC & 51 & PF-05212384/Paclitaxel/Carboplatin & $|/| \mid$ & Recruiting & NCT02920450 \\
\hline $\begin{array}{l}\text { Breast Cancer; NSCLC; Ovary Cancer; } \\
\text { Endometrial Cancer; SCLC }\end{array}$ & 40 & PF-05212384 + Paclitaxel+Carboplatin & । & Recruiting & NCT02069158 \\
\hline ER+/HER2- Breast Cancer & 18 & PF-05212384 + Fulvestrant+ Palbociclib & । & Recruiting & NCT02626507 \\
\hline Breast Cancer & 80 & Hydrpxychloroquine+/- PF-05212384 & $|/| \mid$ & $\begin{array}{l}\text { Not yet } \\
\text { recruiting }\end{array}$ & NCT03400254 \\
\hline Breast Cancer & 120 & $\begin{array}{l}\text { PF-05212384 + Palbociclib+/-Letrozole/ } \\
\text { Fulvestrant }\end{array}$ & I & Recruiting & NCT02684032 \\
\hline Triple Negative Breast Cancer; & 18 & PF-05212384 + PTK7-ADC & I & Recruiting & NCT03243331 \\
\hline
\end{tabular}

Metastatic Breast Cancer 
Table 2 Important ongoing clinical trials with Pl3K-targeted therapies (Continued)

\begin{tabular}{|c|c|c|c|c|c|}
\hline Conditions & $\begin{array}{l}\text { Sample } \\
\text { size }\end{array}$ & Design & Phase & Status & Trial number \\
\hline \multicolumn{6}{|l|}{ PQR309 (Bimiralisib) Dual PI3K/mTOR inhibitor } \\
\hline Lymphoma & 72 & PQR309 & $|/| \mid$ & Recruiting & NCT02249429 \\
\hline Lymphoma; Non-Hodgkin Lymphoma & 72 & PQR309 & $\|$ & Recruiting & NCT03127020 \\
\hline Primary Central Nervous System Lymphoma & 21 & PQR309 & $\|$ & $\begin{array}{l}\text { Not yet } \\
\text { recruiting }\end{array}$ & NCT03120000 \\
\hline Metastatic Breast Cancer & 60 & PQR309 + Eribulin & $|/| \mid$ & Recruiting & NCT02723877 \\
\hline \multicolumn{6}{|l|}{ P7170 Dual PI3K/mTOR inhibitor } \\
\hline Advanced Refractory Solid Tumors & 60 & P7170 & । & Suspended & NCT01762410 \\
\hline \multicolumn{6}{|l|}{ SF-1126 Dual PI3K/mTOR inhibitor } \\
\hline Advanced Hepatocellular Carcinoma & 14 & SF-1126 & । & Recruiting & NCT03059147 \\
\hline $\begin{array}{l}\text { Advanced Castrate-resistant Prostate Cancer; } \\
\text { Squamous NSCLC; Triple Negative Breast Cancer }\end{array}$ & 180 & AZD8186+/-Abiraterone Acetate/AZD2014 & 1 & Recruiting & NCT01884285 \\
\hline \multicolumn{6}{|l|}{ Copanlisib (BAY 80-6946) PI3Kס/a inhibitor } \\
\hline $\begin{array}{l}\text { Recurrent Endometrial, Ovarian, Primary } \\
\text { Peritoneal, or Fallopian Tube Cancer }\end{array}$ & 44 & Copanlisib+Niraparib & 1 & $\begin{array}{l}\text { Not yet } \\
\text { recruiting }\end{array}$ & NCT03586661 \\
\hline Head and Neck Squamous Cell Carcinomas & 32 & Copanlisib+Cetuximab & $|/| \mid$ & Recruiting & NCT02822482 \\
\hline Endometrial cancer & 84 & Copanlisib & $\|$ & Suspended & NCT02728258 \\
\hline HR+, HER2-, Stage I-IV Breast Cancer & 102 & Copanlisib+Letrozole+/-Palbociclib & $|/| \mid$ & Recruiting & NCT03128619 \\
\hline HER2+ Breast Cancer & 19 & Copanlisib +Trastuzumab & 1 & Recruiting & NCT02705859 \\
\hline Non-Hodgkin Lymphoma & 25 & Copanlisib & $|/| \mid$ & $\begin{array}{l}\text { Active not } \\
\text { recruiting }\end{array}$ & NCT02342665 \\
\hline Mature T-Cell and NK-Cell Neoplasm & 36 & Copanlisib+Gemcitabine & $|/| \mid$ & Recruiting & NCT03052933 \\
\hline Advanced or Metastatic Solid Tumor & 65 & Copanlisib+Rogaratinib & । & Recruiting & NCT03517956 \\
\hline Medical Oncology & 51 & Copanlisib+/-Itraconazole/ Rifampin & 1 & $\begin{array}{l}\text { Active not } \\
\text { recruiting }\end{array}$ & NCT02253420 \\
\hline Mixed Tumor, Malignant & 130 & Copanlisib & $|/| \mid$ & Recruiting & NCT03458728 \\
\hline $\begin{array}{l}\text { Biliary Carcinoma; Gall Bladder Carcinoma; } \\
\text { Cholangiocarcinoma; Gastrointestinal Tumor }\end{array}$ & 25 & Copanlisib+Gemcitabine+Cisplatin & $\|$ & Recruiting & NCT02631590 \\
\hline $\begin{array}{l}\text { Refractory/Recurrent Primary Central } \\
\text { Nervous System Lymphoma }\end{array}$ & 45 & Copanlisib+lbrutinib & $|/| \mid$ & $\begin{array}{l}\text { Not yet } \\
\text { recruiting }\end{array}$ & NCT03581942 \\
\hline Marginal Zone Lymphoma & 56 & Copanlisib+Rituximab & $\|$ & $\begin{array}{l}\text { Not yet } \\
\text { recruiting }\end{array}$ & NCT03474744 \\
\hline Large B-Cell Lymphoma & 99 & Copanlisib+Nivolumab & $\|$ & $\begin{array}{l}\text { Not yet } \\
\text { recruiting }\end{array}$ & NCT03484819 \\
\hline $\begin{array}{l}\text { Ann Arbor Stage III/IV Lymphoma; } \\
\text { Metastatic Malignant; Solid Neoplasm }\end{array}$ & 50 & Copanlisib+Nivolumab & 1 & Recruiting & NCT03502733 \\
\hline Non-Hodgkin Lymphoma & 450 & Rituximab+Copanlisib/Placebo & III & Recruiting & NCT02367040 \\
\hline Non-Hodgkin Lymphoma & 227 & Copanlisib & $\|$ & $\begin{array}{l}\text { Active not } \\
\text { recruiting }\end{array}$ & NCT01660451 \\
\hline Non-Hodgkin Lymphoma & 25 & Copanlisib & III & $\begin{array}{l}\text { Active not } \\
\text { recruiting }\end{array}$ & NCT02369016 \\
\hline Non-Hodgkin Lymphoma & 12 & Copanlisib & I & Recruiting & NCT03498430 \\
\hline Non-Hodgkin Lymphoma & 546 & Standard Immunochemotherapy+/- Copanlisib & III & Recruiting & NCT02626455 \\
\hline \multicolumn{6}{|l|}{ Buparlisib (BKM120 NVP-BKM120) Class I PI3K inhibitor } \\
\hline $\begin{array}{l}\text { Metastatic Transitional Cell Carcinoma } \\
\text { of the Urothelium }\end{array}$ & 35 & Buparlisib & $\|$ & $\begin{array}{l}\text { Active not } \\
\text { recruiting }\end{array}$ & NCT01551030 \\
\hline $\begin{array}{l}\text { Metastatic Squamous Neck Cancer With } \\
\text { Occult Primary Squamous Cell Carcinoma; }\end{array}$ & 30 & Buparlisib+Cetuximab & $|/| \mid$ & $\begin{array}{l}\text { Active not } \\
\text { recruiting }\end{array}$ & NCT01816984 \\
\hline
\end{tabular}


Table 2 Important ongoing clinical trials with PI3K-targeted therapies (Continued)

\begin{tabular}{|c|c|c|c|c|c|}
\hline Conditions & $\begin{array}{l}\text { Sample } \\
\text { size }\end{array}$ & Design & Phase & Status & Trial number \\
\hline Head and Neck Cancer & 170 & Buparlisib & $\|$ & Recruiting & NCT01737450 \\
\hline NSCLC & 37 & Buparlisib+Erlotinib & $\|$ & $\begin{array}{l}\text { Active not } \\
\text { recruiting }\end{array}$ & NCT01487265 \\
\hline NSCLC & 38 & Buparlisib+Gefitinib & & $\begin{array}{l}\text { Active not } \\
\text { recruiting }\end{array}$ & NCT01570296 \\
\hline $\begin{array}{l}\text { Advanced Squamous Cell Cancer } \\
\text { of Head and Neck }\end{array}$ & 23 & $\begin{array}{l}\text { Radiotherapy+Buparlisib+ } \\
\text { Cisplatin }\end{array}$ & । & $\begin{array}{l}\text { Active not } \\
\text { recruiting }\end{array}$ & NCT02113878 \\
\hline Breast Cancer & 106 & Buparlisib+lapatinib & $|/| \mid$ & $\begin{array}{l}\text { Suspended } \\
\text { (Data analysis) }\end{array}$ & NCT01589861 \\
\hline Breast Cancer & 1149 & Buparlisib/Placebo+Fulvestrant & III & $\begin{array}{l}\text { Active not } \\
\text { recruiting }\end{array}$ & NCT01610284 \\
\hline Breast Cancer & 110 & Buparlisib & $\|$ & $\begin{array}{l}\text { Active not } \\
\text { recruiting }\end{array}$ & NCT01790932 \\
\hline Metastatic Breast Cancer & 47 & $\begin{array}{l}\text { Buparlisib+Capecitabine+/- } \\
\text { (Trastuzumab/Lapatinib) OR } \\
\text { BYL719+ Capecitabine }\end{array}$ & । & $\begin{array}{l}\text { Active not } \\
\text { recruiting }\end{array}$ & NCT01300962 \\
\hline Breast Cancer Patients With Brain Metastases & 10 & Buparlisib/Capecitabine & $\|$ & $\begin{array}{l}\text { Active not } \\
\text { recruiting }\end{array}$ & NCT02000882 \\
\hline Pre-menopausal Breast Cancer & 40 & $\begin{array}{l}\text { Buparlisib/BYL719 + Tamoxifen+ } \\
\text { Goserelin Acetate }\end{array}$ & 1 & $\begin{array}{l}\text { Active not } \\
\text { recruiting }\end{array}$ & NCT02058381 \\
\hline Ovarian Cancer; Breast Cancer & 118 & Buparlisib/BYL719+ Olaparib & 1 & $\begin{array}{l}\text { Active not } \\
\text { recruiting }\end{array}$ & NCT01623349 \\
\hline Glioblastoma Multiforme & 88 & Buparlisib+Bevacizumab & $|/| \mid$ & $\begin{array}{l}\text { Active not } \\
\text { recruiting }\end{array}$ & NCT01349660 \\
\hline Glioblastoma & 65 & Buparlisib+/-Surgery & $\|$ & $\begin{array}{l}\text { Active not } \\
\text { recruiting }\end{array}$ & NCT01339052 \\
\hline Thyroid Cancers & 47 & Buparlisib & $\|$ & $\begin{array}{l}\text { Active not } \\
\text { recruiting }\end{array}$ & NCT01830504 \\
\hline Thymoma & 14 & Buparlisib & $\|$ & $\begin{array}{l}\text { Active not } \\
\text { recruiting }\end{array}$ & NCT02220855 \\
\hline Malignant Melanoma; Metastases & 22 & Buparlisib & $\|$ & Recruiting & NCT02452294 \\
\hline Melanoma & 140 & $\begin{array}{l}\text { LGX818 + MEK162+/-(Buparlisib/ } \\
\text { LEE011/ BGJ398/ INC280) }\end{array}$ & $\|$ & $\begin{array}{l}\text { Active not } \\
\text { recruiting }\end{array}$ & NCT02159066 \\
\hline Metastatic Colorectal Cancer & 22 & Buparlisib+Panitumumab & $|/| \mid$ & $\begin{array}{l}\text { Active not } \\
\text { recruiting }\end{array}$ & NCT01591421 \\
\hline $\begin{array}{l}\text { Relapsed or Refractory Indolent } \\
\text { B-Cell Lymphoma }\end{array}$ & 18 & Buparlisib+Rituximab & I & $\begin{array}{l}\text { Active not } \\
\text { recruiting }\end{array}$ & NCT02049541 \\
\hline Chronic Lymphocytic Leukemia & 14 & Buparlisib & $\|$ & $\begin{array}{l}\text { Active not } \\
\text { recruiting }\end{array}$ & NCT02340780 \\
\hline $\begin{array}{l}\text { Recurrent/ Refractory Chronic Lymphocytic } \\
\text { Leukemia; Recurrent/ Refractory Small } \\
\text { Lymphocytic Lymphoma }\end{array}$ & 1 & Buparlisib+Ofatumumab/lbrutinib & I & $\begin{array}{l}\text { Active not } \\
\text { recruiting }\end{array}$ & NCT02614508 \\
\hline $\begin{array}{l}\text { Mantle Cell Lymphoma; Follicular } \\
\text { Lymphoma; Diffuse Large B Cell Lymphoma }\end{array}$ & 37 & Buparlisib+lbrutinib & 1 & $\begin{array}{l}\text { Active not } \\
\text { recruiting }\end{array}$ & NCT02756247 \\
\hline \multicolumn{6}{|l|}{ Duvelisib (IPI-145) PI3K $/ Y$ inhibitor } \\
\hline Indolent Non-Hodgkin Lymphoma & 129 & Duvelisib & $\|$ & $\begin{array}{l}\text { Active not } \\
\text { recruiting }\end{array}$ & NCT01882803 \\
\hline Relapsed/Refractory T-cell Lymphomas & 88 & Duvelisib+Romidepsin/ Bortezomib & I & Recruiting & NCT02783625 \\
\hline Peripheral T-cell Lymphoma & 120 & Duvelisib & $\|$ & Recruiting & NCT03372057 \\
\hline Chronic Lymphocytic Leukemia & 47 & Duvelisib+Venetoclax & $|/| \mid$ & Recruiting & NCT03534323 \\
\hline Hematologic Malignancy & 500 & Duvelisib & $\|$ & $\begin{array}{l}\text { Active not } \\
\text { recruiting }\end{array}$ & NCT02711852 \\
\hline
\end{tabular}


Table 2 Important ongoing clinical trials with PI3K-targeted therapies (Continued)

\begin{tabular}{|c|c|c|c|c|c|}
\hline Conditions & $\begin{array}{l}\text { Sample } \\
\text { size }\end{array}$ & Design & Phase & Status & Trial number \\
\hline $\begin{array}{l}\text { Chronic Lymphocytic Leukemia; } \\
\text { Small Lymphocytic Lymphoma }\end{array}$ & 150 & Duvelisib VS Ofatumumab & III & $\begin{array}{l}\text { - Enrolling by } \\
\text { invitation }\end{array}$ & NCT02049515 \\
\hline $\begin{array}{l}\text { Chronic Lymphocytic Leukemia; } \\
\text { Small Lymphocytic Lymphoma }\end{array}$ & 300 & Duvelisib VS Ofatumumab & III & $\begin{array}{l}\text { Active not } \\
\text { recruiting }\end{array}$ & NCT02004522 \\
\hline Chronic Lymphocytic & 50 & Duvelisib & $\|$ & Recruiting & NCT03370185 \\
\hline \multicolumn{6}{|l|}{ Leukemia; Small Lymphocytic Lymphoma } \\
\hline Chronic Lymphocytic Leukemia & 32 & $\begin{array}{l}\text { Duvelisib+Fludarabine+Cyclophosphamide+ } \\
\text { Rituximab }\end{array}$ & $|/| \mid$ & $\begin{array}{l}\text { Active not } \\
\text { recruiting }\end{array}$ & NCT02158091 \\
\hline \multicolumn{6}{|l|}{ RP6530 (Tenalisib) PI3K $/ Y$ inhibitor } \\
\hline $\begin{array}{l}\text { Peripheral T-Cell Lymphoma; Cutaneous } \\
\text { T-Cell Lymphoma }\end{array}$ & 58 & RP6530 & । & $\begin{array}{l}\text { Active not } \\
\text { recruiting }\end{array}$ & NCT02567656 \\
\hline Classical Hodgkin Lymphoma & 57 & RP6530 + Pembrolizumab & 1 & Recruiting & NCT03471351 \\
\hline \multicolumn{6}{|l|}{ Taselisib (GDC-0032) PI3Ka/ $/ / Y$ inhibitor } \\
\hline $\begin{array}{l}\text { Recurrent/ Stage IV Squamous } \\
\text { Cell Lung Carcinoma }\end{array}$ & 59 & Taselisib & $\|$ & $\begin{array}{l}\text { Active not } \\
\text { recruiting }\end{array}$ & NCT02785913 \\
\hline $\begin{array}{l}\text { Metastatic Breast Cancer; Recurrent } \\
\text { Breast Cancer }\end{array}$ & 76 & $\begin{array}{l}\text { Taselisib+Trastuzumab emtansine }+ \text { / } \\
\text { - Pertuzumab OR } \\
\text { Pertuzumab+Trastuzumab+/- Paclitaxel }\end{array}$ & 1 & Recruiting & NCT02390427 \\
\hline $\begin{array}{l}\text { Androgen Receptor Positive Triple } \\
\text { Negative Metastatic Breast Cancer }\end{array}$ & 73 & Taselisib+Enzalutamide & $|/| \mid$ & $\begin{array}{l}\text { Active not } \\
\text { recruiting }\end{array}$ & NCT02457910 \\
\hline Breast Cancer & 290 & Tamoxifen+ Taselisib/Placebo & $|/| \mid$ & Recruiting & NCT02285179 \\
\hline Breast Cancer & 631 & Fulvestrant+ Taselisib/Placebo & III & $\begin{array}{l}\text { Active not } \\
\text { recruiting }\end{array}$ & NCT02340221 \\
\hline PIK3CA-Related Overgrowth & 30 & Taselisib & $|/| \mid$ & Recruiting & NCT03290092 \\
\hline Solid Cancers; Non-Hodgkin Lymphoma & 724 & $\begin{array}{l}\text { Taselisib+/-Fulvestrant/Letrozole/ } \\
\text { Midazolam/ Fulvestrant }\end{array}$ & 1 & $\begin{array}{l}\text { Active not } \\
\text { recruiting }\end{array}$ & NCT01296555 \\
\hline $\begin{array}{l}\text { Advanced Refractory Solid Tumors; } \\
\text { Lymphomas; Multiple Myeloma }\end{array}$ & 6452 & $\begin{array}{l}\text { Molecular Analysis for Therapy Choice } \\
\text { Screening Trial }\end{array}$ & $\|$ & Recruiting & NCT02465060 \\
\hline \multicolumn{6}{|l|}{ KA2237 PI3Kß/Y inhibitor } \\
\hline B Cell Lymphoma & 53 & KA2237 & । & Recruiting & NCT02679196 \\
\hline \multicolumn{6}{|l|}{ BYL719 (Alpelisib) PI3Ka inhibitor } \\
\hline PIK3CA Mutated Advanced Breast Cancer & 90 & BYL719 VS Chemotherapy & $\|$ & Recruiting & NCT03386162 \\
\hline Breast Cancer & 23 & BYL719 + LM716+ Trastuzumab & 1 & $\begin{array}{l}\text { Active not } \\
\text { recruiting }\end{array}$ & NCT02167854 \\
\hline Breast Cancer & 44 & BYL719 + Nab-Paclitaxel & $|/| \mid$ & $\begin{array}{l}\text { Active not } \\
\text { recruiting }\end{array}$ & NCT02379247 \\
\hline HER2+ Metastatic Breast Cancer & 17 & BYL719 + Ado-Trastuzumab Emtansine & 1 & $\begin{array}{l}\text { Active not } \\
\text { recruiting }\end{array}$ & NCT02038010 \\
\hline Metastatic Breast Cancer & 34 & BYL719 & $\|$ & Recruiting & NCT02506556 \\
\hline Malignant Neoplasm of Breast & 28 & BYL719 + Enzalutamide & । & $\begin{array}{l}\text { Not yet } \\
\text { recruiting }\end{array}$ & NCT03207529 \\
\hline Pancreatic Cancer & 15 & BYL719 + Gemcitabine+(Nab)-Paclitaxel & I & $\begin{array}{l}\text { Active not } \\
\text { recruiting }\end{array}$ & NCT02155088 \\
\hline Breast Cancer & 572 & Fulvestrant+ BYL719/Placebo & III & $\begin{array}{l}\text { Active not } \\
\text { recruiting }\end{array}$ & NCT02437318 \\
\hline $\begin{array}{l}\text { Premenopausal Patients With HRt, } \\
\text { HER2- Locally Advanced or Metastatic } \\
\text { Breast Cancer }\end{array}$ & 40 & $\begin{array}{l}\text { BYL719/BKM120+ Tamoxifen+ } \\
\text { Goserelin Acetate }\end{array}$ & । & $\begin{array}{l}\text { Active not } \\
\text { recruiting }\end{array}$ & NCT02058381 \\
\hline Advanced or Metastatic ER+ Breast Cancer & 312 & LSZ102+/- LEE011/BYL719 & । & Recruiting & NCT02734615 \\
\hline $\begin{array}{l}\text { Metastatic or Locally-advanced } \\
\text { Unresectable Breast Cancer }\end{array}$ & 52 & BYL719 + Letrozole/Exemestane & । & $\begin{array}{l}\text { Active not } \\
\text { recruiting }\end{array}$ & NCT01870505 \\
\hline
\end{tabular}


Table 2 Important ongoing clinical trials with PI3K-targeted therapies (Continued)

\begin{tabular}{|c|c|c|c|c|c|}
\hline Conditions & $\begin{array}{l}\text { Sample } \\
\text { size }\end{array}$ & Design & Phase & Status & Trial number \\
\hline Breast Cancer & 160 & BYL-719+ Fulvestrant/Letrozole & $\|$ & Recruiting & NCT03056755 \\
\hline $\begin{array}{l}\text { ER+ Breast Cancer; HER2-negative Breast } \\
\text { Cancer; Invasive Ductal Breast Carcinoma }\end{array}$ & 46 & BYL719 + Letrozole & । & $\begin{array}{l}\text { Active not } \\
\text { recruiting }\end{array}$ & NCT01791478 \\
\hline Breast Cancer & 253 & Letrozole+BYL719/LEE011/ Both & I & Recruiting & NCT01872260 \\
\hline Metastatic Breast Cancer & 47 & $\begin{array}{l}\text { BMK120 + Capecitabine+/- Trastuzumab/ } \\
\text { Lapatinib OR BYL719+ Capecitabine }\end{array}$ & I & $\begin{array}{l}\text { Active not } \\
\text { recruiting }\end{array}$ & NCT01300962 \\
\hline $\begin{array}{l}\text { Head and Neck Cancer and Esophageal } \\
\text { Cancer Patient }\end{array}$ & 259 & $\begin{array}{l}\text { BYL719/Poziotinib/Nintedanib/Abemaciclib/ } \\
\text { (Durvalumab+Tremelimumab) }\end{array}$ & $\|$ & Recruiting & NCT03292250 \\
\hline Head and Neck Squamous Cell Cancer & 30 & BYL719 & N/A & Recruiting & NCT03138070 \\
\hline $\begin{array}{l}\text { Recurrent or Metastatic Squamous } \\
\text { Cell Carcinoma of Head and Neck }\end{array}$ & 43 & BYL719 & $\|$ & Recruiting & NCT02145312 \\
\hline Head and Neck Squamous Cell Cancer & 16 & $\begin{array}{l}\text { BYL719+ Cetuximab+IMRT } \\
\text { (Intensity-Modulated Radiation Therapy) }\end{array}$ & 1 & $\begin{array}{l}\text { Active not } \\
\text { recruiting }\end{array}$ & NCT02282371 \\
\hline $\begin{array}{l}\text { Locoregionally Advanced Squamous } \\
\text { Cell Carcinoma of Head and Neck }\end{array}$ & 36 & $\begin{array}{l}\text { BYL719 + Cisplatin+Radiation } \\
\text { (Intensity modulated radiation therapy) }\end{array}$ & 1 & Recruiting & NCT02537223 \\
\hline Uveal Melanoma & 30 & BYL719+ AEB071 & 1 & $\begin{array}{l}\text { Active not } \\
\text { recruiting }\end{array}$ & NCT02273219 \\
\hline Rectal Cancer & 24 & BYL719 + Capecitabine+Radiation & 1 & Recruiting & NCT02550743 \\
\hline Colorectal Cancer & 150 & LGX818 + Cetuximab+/- BYL719 & $|/| \mid$ & $\begin{array}{l}\text { Active not } \\
\text { recruiting }\end{array}$ & NCT01719380 \\
\hline Patients With Gastrointestinal Stromal Tumor & 56 & BYL719 + ST571 & 1 & $\begin{array}{l}\text { Active not } \\
\text { recruiting }\end{array}$ & NCT01735968 \\
\hline $\begin{array}{l}\text { Adenocarcinoma Lung Cancer; } \\
\text { Squamous Cell Lung Carcinoma }\end{array}$ & 67 & BYL719/AUY922/INC280/LDK378/MEK162 & $\|$ & $\begin{array}{l}\text { Active not } \\
\text { recruiting }\end{array}$ & NCT02276027 \\
\hline $\begin{array}{l}\text { CDKN2A-p16+; Human Papillomavirus+ } \\
\text { Oropharyngeal Squamous Cell Carcinoma }\end{array}$ & 14 & BYL719+ Surgery & $\|$ & $\begin{array}{l}\text { Not yet } \\
\text { recruiting }\end{array}$ & NCT03601507 \\
\hline $\begin{array}{l}\text { Breast Neoplasms; Kidney Neoplasms; } \\
\text { Pancreatic Neuroendocine Neoplasms }\end{array}$ & 79 & BYL719 + Everolimus/Exemestane/Both & 1 & $\begin{array}{l}\text { Active not } \\
\text { recruiting }\end{array}$ & NCT02077933 \\
\hline $\begin{array}{l}\text { Advanced Solid Tumors With an Alteration } \\
\text { of the PIK3CA Gene; ER+ Breast Cancer }\end{array}$ & 221 & BYL719+/-Fulvestrant & I & $\begin{array}{l}\text { Active not } \\
\text { recruiting }\end{array}$ & NCT01219699 \\
\hline Solid Tumors & 41 & BYL719 + Cisplatin & 1 & Recruiting & NCT02620839 \\
\hline Ovarian Cancer; Breast Cancer & 118 & Olaparib+BYL719/BKM120 & 1 & $\begin{array}{l}\text { Active not } \\
\text { recruiting }\end{array}$ & NCT01623349 \\
\hline Meningioma & 25 & BYL719 + Trametinib & 1 & $\begin{array}{l}\text { Not yet } \\
\text { recruiting }\end{array}$ & NCT03631953 \\
\hline \multicolumn{6}{|l|}{ CAL-101 (GS-1101, Idelalisib) PI3Kס inhibitor } \\
\hline Metastasis/Recurrence NSCLC & 40 & CAL-101 + Pembrolizumab & $|/| \mid$ & Recruiting & NCT03257722 \\
\hline Waldenstrom Macroglobulinemia & 50 & Obinutuzumab & $\|$ & $\begin{array}{l}\text { Active not } \\
\text { recruiting }\end{array}$ & NCT02962401 \\
\hline Chronic Lymphocytic Leucemia & 62 & CAL-101 + Bendamustine+GA101 & $\|$ & Recruiting & NCT02445131 \\
\hline $\begin{array}{l}\text { Chronic Lymphocytic LeukemiaSmall } \\
\text { Lymphocytic Lymphoma }\end{array}$ & 50 & CAL-101 + Ofatumumab & $\|$ & Suspended & NCT02135133 \\
\hline Follicular Non-Hodgkin Lymphoma Refractory & 260 & CAL-101 & N/A & Recruiting & NCT03568929 \\
\hline Chronic Lymphocytic Leukemia & 42 & CAL-101 + Rituximab+Venetoclax & । & $\begin{array}{l}\text { Not yet } \\
\text { recruiting }\end{array}$ & NCT03639324 \\
\hline Chronic Lymphocytic Leukemia & 104 & CAL-101 + Rituximab & N/A & $\begin{array}{l}\text { Not yet } \\
\text { recruiting }\end{array}$ & NCT03545035 \\
\hline Diffuse Large B-Cell & 36 & CAL-101 + (Rituximab+lfosfa & 1 & Recruiting & NCT03349346 \\
\hline Lymphoma; Mediastinal B-cell Lymphoma & & mide+Carboplatin+Etoposide) (RICE) & & & \\
\hline
\end{tabular}


Table 2 Important ongoing clinical trials with PI3K-targeted therapies (Continued)

\begin{tabular}{|c|c|c|c|c|c|}
\hline Conditions & $\begin{array}{l}\text { Sample } \\
\text { size }\end{array}$ & Design & Phase & Status & Trial number \\
\hline Chronic Lymphocytic Leukemia & 35 & CAL-101 + Tirabrutinib+/- Obinutuzumab & $\|$ & Recruiting & NCT02968563 \\
\hline $\begin{array}{l}\text { Chronic Lymphocytic Leukemia; } \\
\text { Small Lymphocytic Lymphoma }\end{array}$ & 24 & MOR00208 + CAL-101/Venetoclax & $\|$ & Recruiting & NCT02639910 \\
\hline Chronic Lymphocytic Leukemia & 308 & $\begin{array}{l}\text { Acalabrutinib VS Rituximab + CAL- } \\
\text { 101/Bendamustine }\end{array}$ & III & Recruiting & NCT02970318 \\
\hline $\begin{array}{l}\text { Recurrent Chronic Lymphocytic Leukemia; } \\
\text { Extranodal Marginal Zone Lymphoma; } \\
\text { Follicular Lymphoma }\end{array}$ & 68 & Pembrolizumab+/-CAL-101//brutinib & $\|$ & Recruiting & NCT02332980 \\
\hline B-cell Malignancies & 197 & $\begin{array}{l}\text { Tirabrutinib+/-CAL-101/Entospletinib+/- } \\
\text { Obinutuzumab }\end{array}$ & I & $\begin{array}{l}\text { Active not } \\
\text { recruiting }\end{array}$ & NCT02457598 \\
\hline $\begin{array}{l}\text { Chronic Lymphocytic Leukemia; Peripheral } \\
\text { T-cell Lymphoma }\end{array}$ & 123 & $\begin{array}{l}\text { TRU-016 + Rituximab/ Obinutuzumab/ } \\
\text { lbrutinib/ Bendamustine OR TRU-016 + } \\
\text { Rituximab+CAL-101 }\end{array}$ & 1 & Recruiting & NCT01644253 \\
\hline $\begin{array}{l}\text { Acute Lymphoblastic Leukemia; Acute } \\
\text { Myeloid Leukemia }\end{array}$ & 24 & $\begin{array}{l}\text { Personalized Kinase Inhibitor Therapy } \\
\text { Combined With Chemotherapy }\end{array}$ & 1 & Recruiting & NCT02779283 \\
\hline Non-Hodgkin Lymphoma & 30 & CAL-101 & N/A & Recruiting & NCT02928510 \\
\hline Hematological Malignancies & 150 & CAL-101 VS Ibrutinib (Side Effects) & N/A & Recruiting & NCT02824159 \\
\hline Recurrent Chronic Lymphoid Leukemia & 3 & ACY-1215+ CAL-101/lbrutinib & 1 & $\begin{array}{l}\text { Active not } \\
\text { recruiting }\end{array}$ & NCT02787369 \\
\hline Chronic Lymphocytic Leukemia & 416 & Rituximab+Bendamustine+ Placebo/ CAL-101 & III & $\begin{array}{l}\text { Active not } \\
\text { recruiting }\end{array}$ & NCT01569295 \\
\hline Follicular Lymphoma & 240 & CAL-101 & III & Recruiting & NCT02536300 \\
\hline $\begin{array}{l}\text { B Cells-Tumors; B Cell Chronic Lymphocytic } \\
\text { Leukemia; Follicular Lymphoma; Mantle Cell } \\
\text { Lymphoma; Large B-Cell Diffuse Lymphoma }\end{array}$ & 60 & CAL-101 VS Placebo & 1 & Recruiting & NCT03151057 \\
\hline $\begin{array}{l}\text { Chronic Lymphocytic Leukemia; } \\
\text { Small Lymphocytic Lymphoma }\end{array}$ & 24 & MOR00208 + CAL-101/ Venetoclax & $\|$ & $\begin{array}{l}\text { Active not } \\
\text { recruiting }\end{array}$ & NCT02639910 \\
\hline Diffuse Large B Cell Lymphoma & 72 & CAL-101 & $\|$ & Recruiting & NCT03576443 \\
\hline B-Cell Non-Hodgkin Lymphoma & 34 & CAL-101 & $\|$ & Recruiting & NCT03133221 \\
\hline Chronic Lymphocytic Leukemia & 20 & CAL-101 + Rituximab & N/A & Recruiting & NCT02993536 \\
\hline Chronic Lymphocytic Leukaemia & 150 & CAL-101 + Rituximab & N/A & $\begin{array}{l}\text { Not yet } \\
\text { recruiting }\end{array}$ & NCT03582098 \\
\hline \multicolumn{6}{|l|}{ GSK2636771 PI3K $\beta$ inhibitor } \\
\hline Gastric Cancer & 400 & Biomarker Screening & N/A & Recruiting & NCT02951091 \\
\hline Advanced Gastric Adenocarcinoma & 66 & GSK2636771+ Paclitaxel & $|/| \mid$ & Recruiting & NCT02615730 \\
\hline Metastatic Castration-Resistant Prostate Cancer & 64 & GSK2636771+ Enzalutamide & 1 & Recruiting & NCT02215096 \\
\hline $\begin{array}{l}\text { Melanoma and Other Malignant } \\
\text { Neoplasms of Skin; Metastatic Melanoma }\end{array}$ & 41 & GSK2636771 + Pembrolizumab & $|/| \mid$ & Recruiting & NCT03131908 \\
\hline Advanced Malignant Solid Neoplasm & - & $\begin{array}{l}\text { Patients with PTEN mutation, deletion, } \\
\text { expression or loss were given GSK2636771 }\end{array}$ & $\|$ & Recruiting & NCT02465060 \\
\hline \multicolumn{6}{|l|}{ INCB050465 (Parsaclisib) PI3Kס inhibitor } \\
\hline MPN (Myeloproliferative Neoplasms) & 78 & INCB050465 + Ruxolitinib & $\|$ & Recruiting & NCT02718300 \\
\hline Advanced Solid Tumors & 237 & Pembrolizumab+ltacitinib/ INCB050465 & 1 & Recruiting & NCT02646748 \\
\hline Advanced Solid Tumors & 159 & Itacitinib+Epacadostat/ INCB050465 & 1 & $\begin{array}{l}\text { Active, not } \\
\text { recruiting }\end{array}$ & NCT02559492 \\
\hline $\begin{array}{l}\text { Solid Tumors; Advanced } \\
\text { Malignancies; Metastatic Cancer }\end{array}$ & 80 & $\begin{array}{l}\text { la:INCB052793 } \\
\text { Ib:INCB052793 + (Gemcitabine+Nab-Paclitaxel+ } \\
\text { Dexamethasone+Carfilzomib/+Bortezomib+ } \\
\text { Lenalidomide+Azacitidine+INCB052793+ } \\
\text { Pomalidomide+INCB050465) } \\
\text { Il:INCB052793 + Azacitidine+ INCB039110 }\end{array}$ & $|/| \mid$ & $\begin{array}{l}\text { Active not } \\
\text { recruiting }\end{array}$ & NCT02265510 \\
\hline
\end{tabular}


Table 2 Important ongoing clinical trials with PI3K-targeted therapies (Continued)

\begin{tabular}{|c|c|c|c|c|c|}
\hline Conditions & $\begin{array}{l}\text { Sample } \\
\text { size }\end{array}$ & Design & Phase & Status & Trial number \\
\hline Unresectable or Metastatic Solid Tumors & 100 & INCMGA00012 + Epacadostat / INCB050465 & । & Recruiting & NCT03589651 \\
\hline Primary Sjögren's Syndrome & 12 & INCB050465 & $\|$ & $\begin{array}{l}\text { Not yet } \\
\text { recruiting }\end{array}$ & NCT03627065 \\
\hline Lymphoma & 120 & INCB050465 & $\|$ & Recruiting & NCT03235544 \\
\hline Lymphoma & 120 & INCB050465+/-CITADEL-204 & $\|$ & Recruiting & NCT03144674 \\
\hline Lymphoma & 60 & INCB050465 & $\|$ & $\begin{array}{l}\text { Active not } \\
\text { recruiting }\end{array}$ & NCT02998476 \\
\hline Lymphoma & 18 & INCB050465 & । & Recruiting & NCT03314922 \\
\hline Lymphoma & 45 & INCB050465 + Bendamustine +Obinutuzumab & 1 & Recruiting & NCT03039114 \\
\hline Lymphoma & 100 & INCB050465 & $\|$ & Recruiting & NCT03126019 \\
\hline B-Cell Malignancies & 88 & $\begin{array}{l}\text { INCB050465+/-Itacitinib OR INCB050465+ } \\
\text { Rituximab+Ifosfamide+Carboplatin+Etoposide }\end{array}$ & $|/| \mid$ & $\begin{array}{l}\text { Active not } \\
\text { recruiting }\end{array}$ & NCT02018861 \\
\hline $\begin{array}{l}\text { Relapsed/ Refractory Diffuse Large } \\
\text { B-Cell Lymphoma }\end{array}$ & 25 & INCB050465 + INCB053914 & 1 & $\begin{array}{l}\text { Not yet } \\
\text { recruiting }\end{array}$ & NCT03688152 \\
\hline B-cell Lymphoma & 81 & $\begin{array}{l}\text { INCB050465 + Rituximab+/- Bendamustine } \\
\text { OR INCB050465+ Ibrutinib }\end{array}$ & 1 & Recruiting & NCT03424122 \\
\hline \multicolumn{6}{|l|}{$\begin{array}{l}\text { Serabelisib (INK-1117,MLN-1117,TAK-117) } \\
\text { PI3Ka inhibitor }\end{array}$} \\
\hline Advanced Solid Tumor & 30 & Serabelisib+TAK-228+ Paclitaxel & 1 & $\begin{array}{l}\text { Not yet } \\
\text { recruiting }\end{array}$ & NCT03154294 \\
\hline $\begin{array}{l}\text { Clear-cell Metastatic } \\
\text { Renal Cell Carcinoma }\end{array}$ & 96 & MLN0128+/-Serabelisib VS Everolimus & $\|$ & $\begin{array}{l}\text { Active not } \\
\text { recruiting }\end{array}$ & NCT02724020 \\
\hline Endometrial Neoplasms & 242 & $\begin{array}{l}\text { Paclitaxel+/- MLN0128 OR MLN0128+/- } \\
\text { Serabelisib }\end{array}$ & $\|$ & Recruiting & NCT02725268 \\
\hline Triple Negative Breast Cancer & 20 & TAK-228 + Serabelisib+ Cisplatin+Nab Paclitaxel & $\|$ & Recruiting & NCT03193853 \\
\hline \multicolumn{6}{|l|}{ ME401 (PWT-143) PI3Kס inhibitor } \\
\hline $\begin{array}{l}\text { Chronic Lymphocytic Leukemia (CLL) } \\
\text { Small Lymphocytic Lymphoma (SLL), } \\
\text { B-cell Non-Hodgkin Lymphoma }\end{array}$ & 133 & ME401+/-Rituximab & 1 & Recruiting & NCT02914938 \\
\hline \multicolumn{6}{|l|}{ Umbralisib (RP5264, TGR-1202) PI3Kס inhibitor } \\
\hline $\begin{array}{l}\text { Marginal Zone Lymphoma; } \\
\text { Waldenstrom Macroglobulinemia }\end{array}$ & 30 & Umbralisib & $\|$ & Recruiting & NCT03364231 \\
\hline Chronic Lymphocytic Leukemia & 30 & Ublituximab +Umbralisib + Venetoclax & $|/| \mid$ & Recruiting & NCT03379051 \\
\hline Follicular Lymphoma & 150 & $\begin{array}{l}\text { Obinutuzumab+ Umbralisib / } \\
\text { lenalidomide/Chemotherapy }\end{array}$ & $\|$ & Recruiting & NCT03269669 \\
\hline $\begin{array}{l}\text { Non-Hodgkin Lymphoma; Chronic } \\
\text { Lymphocytic Leukemia }\end{array}$ & 50 & TG-1701 +/- (Ublituximab + Umbralisib) & । & Recruiting & NCT03671590 \\
\hline $\begin{array}{l}\text { Chronic Lymphocytic Leukemia; } \\
\text { B-cell Non-Hodgkin Lymphoma }\end{array}$ & 36 & Umbralisib +Pembrolizumab & I & Recruiting & NCT03283137 \\
\hline $\begin{array}{l}\text { Chronic Lymphocytic Leukemia/Small } \\
\text { Lymphocytic Lymphoma; Mantle } \\
\text { Cell Lymphoma }\end{array}$ & 45 & Umbralisib+lbrutinib & I & $\begin{array}{l}\text { Active not } \\
\text { recruiting }\end{array}$ & NCT02268851 \\
\hline \multicolumn{6}{|c|}{ CUDC-907 (Fimepinostat) PI3Ka/ $/ \delta$ and HDAC1/2/3/10 inhibitor } \\
\hline Advanced/Relapsed Solid Tumors & 60 & CUDC-907 & I & Recruiting & NCT02307240 \\
\hline $\begin{array}{l}\text { Lymphoma; Neuroblastoma; Brain Tumor; } \\
\text { Solid Tumor }\end{array}$ & 44 & CUDC-907 & I & Recruiting & NCT02909777 \\
\hline Multiple Myeloma; Lymphoma & 88 & CUDC-907 & I & $\begin{array}{l}\text { Active not } \\
\text { recruiting }\end{array}$ & NCT01742988 \\
\hline Relapsed and/or Refractory Diffuse Large & 200 & CUDC-907 & $\|$ & Recruiting & NCT02674750 \\
\hline
\end{tabular}

B-cell Lymphoma Including With

Myc Alterations 
Table 2 Important ongoing clinical trials with PI3K-targeted therapies (Continued)

\begin{tabular}{|c|c|c|c|c|c|}
\hline Conditions & $\begin{array}{l}\text { Sample } \\
\text { size }\end{array}$ & Design & Phase & Status & Trial number \\
\hline \multicolumn{6}{|l|}{ Rigosertib (ON-01910) PI3K and PIk-1 inhibitor } \\
\hline Leukemia; Myelofibrosis; Anemia; Splenomegaly & 35 & Rigosertib & $\|$ & Recruiting & NCT02730884 \\
\hline Myelodysplastic Syndromes & 36 & Rigosertib & । & Suspended & NCT02075034 \\
\hline Myelodysplastic Syndromes & 45 & Rigosertib & $\|$ & $\begin{array}{l}\text { Active not } \\
\text { recruiting }\end{array}$ & NCT01904682 \\
\hline $\begin{array}{l}\text { Myelodysplastic Syndromes; MDS; RAEB; } \\
\text { Chronic Myelomonocytic Leukemia }\end{array}$ & 299 & Rigosertib & III & $\begin{array}{l}\text { Active not } \\
\text { recruiting }\end{array}$ & NCT01241500 \\
\hline $\begin{array}{l}\text { Myelodysplastic Syndromes; Refractory } \\
\text { Anemia With Excess Blasts; Chronic } \\
\text { Myelomonocytic Leukemia; Cytopenia }\end{array}$ & 67 & Rigosertib & III & $\begin{array}{l}\text { Active not } \\
\text { recruiting }\end{array}$ & NCT01928537 \\
\hline Myelodysplastic Syndromes & 12 & Rigosertib & । & Recruiting & NCT03495167 \\
\hline $\begin{array}{l}\text { Myelodysplastic Syndrome; Acute Myeloid } \\
\text { Leukemia; Chronic Myelomonocytic Leukemia }\end{array}$ & 45 & Rigosertib+Azacitidine & |//I & $\begin{array}{l}\text { Active not } \\
\text { recruiting }\end{array}$ & NCT01926587 \\
\hline $\begin{array}{l}\text { Myelodysplastic Syndrome; MDS; } \\
\text { Refractory Anemia With Excess Blasts; RAEB }\end{array}$ & 360 & $\begin{array}{l}\text { Rigosertib VS. Any approved } \\
\text { or standard-of-care therapy }\end{array}$ & III & Recruiting & NCT02562443 \\
\hline
\end{tabular}

Abbreviations: NSCLC Non-small cell lung cancer, SCLC Small cell lung cancer, ER Estrogen Receptor, PR Progesterone receptor

daily was tolerable, but objective anti-tumor responses were not observed in these patients [100]. The most frequent treatment-related AEs in the study population were fatigue, decreased appetite, nausea, hyperglycemia and rash. Maximum tolerated dose (MTD) for PF-05212384 was estimated to be $154 \mathrm{mg}$ weekly in a phase II trial; the most common AEs were mucosal inflammation, stomatitis, nausea, decreased appetite, vomiting and fatigue [101]. Clinical benefits were noted in 11 of 78 patients, with 2 confirmed PR, 1 unconfirmed $\mathrm{PR}$, and 8 long-lasting stable (> 6 months) [101].
A multi-arm phase I study evaluated dose-limiting toxicity, safety, pharmacokinetics and preliminary antitumor activity of the PF-04691502 and PF-05212384 plus irinotecan or the MEK inhibitor PD-0325901 in advanced cancer [102]. In this clinical study, MTD for PF-05212384 plus irinotecan $(180 \mathrm{mg} / \mathrm{m} 2)$ was estimated to be $110 \mathrm{mg}$ weekly, and for PF-05212384 plus PD-0325901 (4 mg BID) was not reached at the highest dose at PF-05212384 154 mg weekly; the PF-04691502 (4 mg/6 mg, QD) combination arms were terminated early due to poor tolerability. Further preliminary evidence of clinical activity was observed in

Table 3 Drugs targeting PI3K in clinical trial

\begin{tabular}{|c|c|c|c|c|c|}
\hline Compound & Terminated & Phase I & Phase II & Phase III & FDA approved \\
\hline $\begin{array}{l}\text { Dual PI3K/mTOR } \\
\text { inhibitor }\end{array}$ & $\begin{array}{l}\text { BGT-226 } \\
\text { (Novartis) } \\
\text { DS-7423 } \\
\text { (Daiichi Sankyo) } \\
\text { PF-04691502 } \\
\text { (Pfizer) } \\
\text { PKI-179 (Pfizer) }\end{array}$ & $\begin{array}{l}\text { GSK458/Omipalisib } \\
\text { (GlaxoSmithKline) } \\
\text { P7170 (Piramal) } \\
\text { SB2343/NS-5584 } \\
\text { (Verastem) }\end{array}$ & $\begin{array}{l}\text { BEZ235/Dactolisib (Novartis) } \\
\text { GDC-0084 (Novogen) } \\
\text { GDC-0980/Apitolisib } \\
\text { (Genentech) } \\
\text { LY3023414 (Eli Lilly) } \\
\text { PQR309/Bimiralisib } \\
\text { (PIQUR Therapeutics) } \\
\text { XL765/Noxtalisib (Sanofi) } \\
\text { SF-1126 (SignalRx) }\end{array}$ & $\begin{array}{l}\text { PF-05212384/ } \\
\text { gedatolisib/ } \\
\text { PKI-587 (Pfizer) }\end{array}$ & \\
\hline $\begin{array}{l}\text { Pan-PI3K } \\
\text { inhibitor }\end{array}$ & $\begin{array}{l}\text { GDC-0941/Pictilisib } \\
\text { (Genentech) } \\
\text { PX-866 (Oncothyreon) } \\
\text { TG100-115 (Sanofi) }\end{array}$ & $\begin{array}{l}\text { CH5132799 } \\
\text { (TohokuNiproPharm) }\end{array}$ & $\begin{array}{l}\text { XL147/ Pilaralisib (Sanofi) } \\
\text { ZSTK474 (Zenyaku Kogyo) }\end{array}$ & $\begin{array}{l}\text { BKM-120/Buparlisib } \\
\text { (Novartis) }\end{array}$ & $\begin{array}{l}\text { BAY80-6946/ } \\
\text { Copanlisib } \\
\text { (Bayer) }\end{array}$ \\
\hline $\begin{array}{l}\text { Isoform-specific } \\
\text { PI3K inhibitor }\end{array}$ & $\begin{array}{l}\text { AZD8835 } \\
\text { (AstraZeneca) } \\
\text { \$/a } \\
\text { WX-037 (Wilex) a }\end{array}$ & $\begin{array}{l}\text { AZD8186 } \\
\text { (AstraZeneca) } \beta / \delta \\
\text { KA2237 (Karus } \\
\text { Therapeutics) } \beta / \delta \\
\text { GS-9820/CAL-120 } \\
\text { (Gilead) } \beta / \delta \\
\text { ME401/PWT-143 } \\
\text { (MEl Pharma) } \delta\end{array}$ & $\begin{array}{l}\text { AMG } 319 \text { (Amgen) } \delta \\
\text { GSK2636771 (GlaxoSmithKline) } \beta \\
\text { INCB050465/Parsaclisib (Incyte) } \delta \\
\text { Serabelisib/INK-1117 (Takeda) a } \\
\text { Umbralisib/TGR-1202 } \\
\text { (TG Therapeutics) } \delta \\
\text { RP6530/Tenalisib(Rhizen } \\
\text { Pharmaceuticals) } \delta / Y\end{array}$ & $\begin{array}{l}\text { GDC-0032/Taselisib } \\
\text { (Genentech) a/ } / / \gamma \\
\text { BYL719/Alpelisib } \\
\text { (Novartis) a }\end{array}$ & $\begin{array}{l}\text { Duvelisib/IPI-145 } \\
\text { (Infinity) } \delta / Y \\
\text { CAL-101/idelalisib } \\
\text { (Gilead) } \delta\end{array}$ \\
\hline Others & & & CUDC-907/Fimepinostat (Curis) & $\begin{array}{l}\text { Rigosertib/ON-01910 } \\
\text { (Onconova Therapeutics) }\end{array}$ & \\
\hline
\end{tabular}




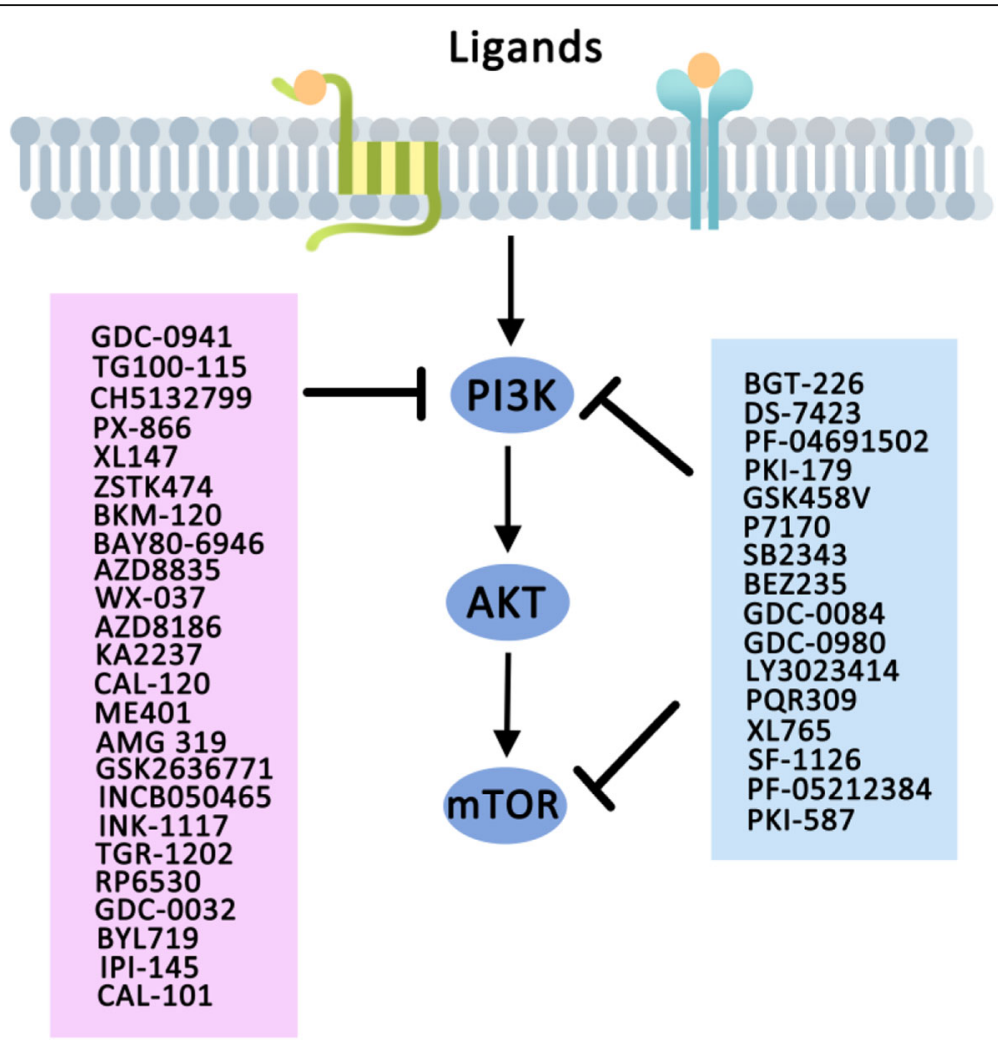

Fig. 2 Targeting PI3K/Akt/mTOR pathway in cancer

PF-05212384 combination arms. Similar results were also reported in a phase II study, which demonstrated poor tolerability of PF-04691502, Whilst also demonstrating acceptable tolerability and moderate anti-tumor activity of PF-05212384 in patients with recurrent endometrial cancer [103]. Ongoing clinical studies are exploring efficacy of PF-05212384 alone and in combination in breast cancer, lung cancer, head and neck cancer, ovary cancer, endometrial cancer, and pancreatic cancer.

\section{Pan-PI3K inhibitors BKM120 (NVP-BKM120, Buparlisib)}

BKM120 (buparlisib) is an orally pan-class I, reversible inhibitor of PI3K. In vitro, buparlisib demonstrates potent antiproliferative effect in human cancer cell lines. In vivo, buparlisib exhibits good oral bioavailability and significant antitumor activity in human tumor xenograft models at tolerated doses [104]. In the first-in-human, phase I, dose-escalation study of buparlisib in western patients with advanced solid tumors, MTD was established at $100 \mathrm{mg}$ daily [105], which was confirmed in the dose-expansion part of another study [106]. The most common treatment-related AEs included rash, hyperglycemia, diarrhea, anorexia, mood alteration, decreased appetite, nausea and abnormal hepatic function $[105,106]$. Phase I studies of buparlisib in Japanese and Chinese patients with advanced solid tumors also established a recommended dose of $100 \mathrm{mg}$ daily [107, 108]. The MTD was $80 \mathrm{mg} / \mathrm{d}$ in a phase I study of buparlisib in patients with advanced acute leukemias [109].

A phase I trial in patients with advanced solid tumors suggested that the MTD of buparlisib in combination with standard doses of mFOLFOX6 (every 2 weeks of a 28-day cycle) was $40 \mathrm{mg}$ daily; increased toxicity was observed compared to that expected from either buparlisib or mFOLFOX6 alone [110]. This trial concluded that further studies of buparlisib in combination with mFOLFOX6 are not recommended in gastrointestinal tumor. In a phase $\mathrm{Ib}$ clinical trial, addition of buparlisib ( $100 \mathrm{mg} /$ day) to carboplatin + paclitaxel was well tolerated in patients with advanced solid tumors [111]. Confirmed objective response was observed in 5 of 25 patients with measurable disease, in particularly, all 3 patients with loss of PTEN expression benefitted clinically from treatment [111]. Interestingly, in the dose expansion study, this combination was revealed to show no significant clinical activity amongst the group of PTEN deficient tumors [112].

In combination with trametinib (MEK inhibitor), buparlisib $60 \mathrm{mg}$ daily plus trametinib $1.5 \mathrm{mg}$ daily displayed promising antitumor activity in patients with KRAS-mutant ovarian cancer, however, modest antitumor activity was observed in patients with non-small cell lung cancer and 
pancreatic cancer [113]. In a phase I dose escalation study, the MTD of combining buparlisib with olaparib (PARP inhibitor) was determined to be BKM120 $50 \mathrm{mg}$ daily and olaparib $300 \mathrm{mg}$ daily. Anticancer activity was observed in patients with breast cancer and ovarian cancer [114]. However, in a phase II study, buparlisib was associated with a poor safety profile and minimal antitumor activity in advanced or recurrent endometrial carcinoma [115]. In patients with metastatic renal cell carcinoma progressing on vascular endothelial growth factor (VEGF) targeted therapies, buparlisib $(80 \mathrm{mg} /$ day $)$ with bevacizumab $(10 \mathrm{mg} / \mathrm{kg}$ every 2 weeks), was shown to be a tolerable regimen with preliminary activity [116]. In patients with castration-resistant prostate cancer, buparlisib did not demonstrate significant activity in a phase II trial, furthermore, the combination of buparlisib with abiraterone acetate was not recommended as a phase Ib study reported [86, 117].

Several clinical trials investigated the use of buparlisib in patients with breast cancer. The combination of buparlisib with capecitabine in patients with metastatic breast cancer was suggested to be well-tolerated in patients with metastatic breast cancer, with 5 of 17 patients demonstrating complete responses (CR) or PR [118]. The combination of buparlisib $(100 \mathrm{mg} /$ day $)$ and trastuzumab $(2 \mathrm{mg} / \mathrm{kg}$ every week) was well tolerated, and preliminary signs of antitumor activity were observed in patients with HER2-positive advanced breast cancer resistant to trastuzumab-based therapy [119]. A randomized adaptive phase II/III study (BELLE-4) suggested that addition of buparlisib to paclitaxel did not improve PFS of patients with HER2 negative advanced breast cancer [120]. In a placebo-controlled phase II trial (NeoPHOEBE), addition of the pan-PI3K inhibitor buparlisib to taxane-trastuzumab-based therapy in HER2 positive early breast cancer was revealed to be unfeasible [121].

Combination trials of buparlisib with endocrine therapy were conducted. MTD was estimated as buparlisib $100 \mathrm{mg}$ daily plus fulvestrant in patients with metastatic estrogen receptor positive breast cancer in a phase I trial [122]. The most common AEs included fatigue, transaminases elevation, rash, and diarrhea. In a phase 3, randomized, placebo-controlled trial (BELLE-2), the addition of buparlisib to fulvestrant significantly prolonged PFS (6.9 vs.5.0 months, HR0.78, one-sided $p=0.00021$ ) compared with the placebo plus fulvestrant group in postmenopausal women with hormone-receptor-positive, HER2-negative, advanced breast cancer [123]. Prespecified exploratory analyses in BELLE-2 showed that the combination regimen resulted in meaningful clinical benefits in the patients with circulating tumor DNA (ctDNA) PIK3CA mutant. Serious AEs were reported in 134 (23\%) of 573 patients in the buparlisib group compared with 90 (16\%) of 570 patients in the placebo group. Based on these findings, BELLE- 3 was to assess the efficacy of buparlisib or placebo in combination with fulvestrant in hormone-receptor-positive, HER2-negative, advanced breast cancer patients with PIK3CA-mutant and wild-type status detected in ctDNA [124]. Buparlisib group was shown to have better PFS than the placebo group (3.9 vs.1.8 months, HR0.67, one-sided $p=0.00030$ ), but serious AEs were more frequently reported in the buparlisib group ( $22 \%$ vs. $16 \%)$.

\section{BAY 80-6946 (Copanlisib)}

BAY 80-6946 (copanlisib) is an intravenous, potent, highly selective and reversible pan-class I PI3K inhibitor with predominant activity against the $\mathrm{p} 110 \alpha$ and $\mathrm{p} 110 \delta$ isoforms, currently in clinical development [125]. The first-in-human phase I study of copanlisib monotherapy in patients with advanced solid tumors and non-Hodgkin lymphomas determined the MTD to be $0.8 \mathrm{mg} / \mathrm{kg}$ (dosed intermittently on days 1,8 , and 15 of a 28 -day cycle), and promising anti-tumor activity was observed, especially in patients with non-Hodgkin lymphoma [126]. The most common treatment-related AEs included nausea and transient hyperglycemia [126]. In a phase I study among Japanese patients with advanced or refractory solid tumor, MTD of $0.8 \mathrm{mg} / \mathrm{kg}$ was also observed; the most frequent AEs were hyperglycemia, hypertension, and constipation [127]. A phase I, dose-escalation study of copanlisib in combination with gemcitabine or cisplatin plus gemcitabine (CisGem) recommended copanlisib $0.8 \mathrm{mg} / \mathrm{kg}$ for patients with advanced cancer. Copanlisib plus CisGem demonstrated favorable clinical response than CisGem [128].

In a phase II study of copanlisib in different subtypes of indolent or aggressive lymphoma, the objective response rate was $44 \%$ (14/32) in indolent lymphoma and 27\% (13/ 48 ) in the aggressive lymphoma. In this trial, enhanced antitumor effects were observed in tumors with upregulated PI3K pathway gene expression [129]. Based on this trial, another phase II trial was conducted with participants suffering from relapsed or refractory indolent B-cell lymphoma; overall response rates (ORR) of 59\% (84/142) and CR rates of $12 \%$ were observed, leading to accelerated approval of copanlisib for relapsed follicular lymphoma [130, 131]. Clinical trials of copanlisib are ongoing, including several phase III trials in patients with non-Hodgkin lymphoma.

\section{IPI-145 (Duvelisib)}

IPI-145 (duvelisib) is an oral dual inhibitor of PI3K- $\delta$ and PI3K- $\gamma$ currently in clinical development. Preclinical studies revealed that IPI-145 causes direct killing in primary chronic lymphocytic leukemia cells in a dose- and time-dependent manner, whereas not bring direct cytotoxicity to normal human B cells [132]. In a phase I, open-label study of duvelisib, the ORR in patients with relapsed/refractory peripheral T-cell lymphoma and cutaneous T-cell lymphoma were 50\% $(8 / 16)$ and $31.6 \%(6 / 19)$ respectively [133]. The most frequently reported AEs were transaminase increases, 
maculopapular rash, and neutropenia. Moreover, a phase II study is planned to further evaluate the efficacy and safety of duvelisib in patients with relapsed and refractory peripheral T-cell lymphoma. The samples of patients with chronic lymphocytic leukemia of this trial were obtained, and the gene-expression studies demonstrated that expression of anti-apoptotic protein BCL2 and several BH3-only pro-apoptotic genes were upregulated on duvelisib therapy [134]. In vitro, the combination of duvelisib and BCL2 inhibitor venetoclax resulted in enhanced apoptosis in chronic lymphocytic leukemia cells [134].

A phase I dose-escalation study in patients with relapsed/ refractory indolent non-Hodgkin lymphoma reported the antitumor activity of duvelisib, with an ORR of $65 \%$ including CR in $25 \%$ of responding patients [135]. The phase II Dynamo study enrolled 129 patients with relapsed/refractory indolent non-Hodgkin lymphoma, and the ORR was $46 \%$, with acceptable safety profile. The response rate across the disease subtypes was 41,68 , and $33 \%$ for patients with follicular lymphoma, small lymphocytic lymphoma, and marginal zone lymphoma, respectively [136]. More recently, in the randomized phase III DUO trial of duvelisib versus ofatumumab monotherapy, patients with relapsed or refractory chronic lymphocytic leukemia/small lymphocytic lymphoma were randomized to oral duvelisib $25 \mathrm{mg}$ BID $(n=160)$ or ofatumumab intravenous $(n=159)$ [137]. Compared with ofatumumab group, patients who received duvelisib were shown to have significantly improving median PFS (13.3 months vs. 9.9 months; HR 0.52; $p<0.0001)$. The ORR was significantly higher with duvelisib $(74 \%$ vs. $45 \%$; $\mathrm{p}<0.0001)$ regardless of $\operatorname{del}(17 \mathrm{p})$ status. In September, 2018, the FDA granted regular approval to duvelisib for the treatment of adult patients with relapsed or refractory chronic lymphocytic leukemia or small lymphocytic lymphoma after at least two prior therapies. In addition, duvelisib received accelerated approval for adult relapsed or refractory follicular lymphoma patients who received at least two prior systemic therapies.

\section{GDC-0941 (Pictilisib)}

GDC-0941 (pictilisib) is a potent, orally class I pan-PI3K inhibitor, which is currently in clinical development [138, 139]. Pictilisib has demonstrated antitumor activity in human tumor xenograft murine models [140, 141]. Pictilisib exhibited favorable tolerability with potential clinical antitumor activity in the first-in-human phase I study of advanced solid tumor, and the MDT was $330 \mathrm{mg} /$ day [142]. The most common drug-related toxicities were nausea, fatigue, diarrhea, vomiting, dysgeusia and decreased appetite [142]. Pictilisib demonstrated a favorable safety profile in Japanese patients with advanced solid tumor or non-squamous non-small cell lung cancer in a phase Ia/Ib study; no objective anti-tumor responses were observed in patients with advanced solid tumor while partial anti-tumor responses were observed in patients with non-squamous non-small cell lung cancer [143]. The MDT was determined to be $340 \mathrm{mg} /$ day for monotherapy and was $260 \mathrm{mg} /$ day for combination with carboplatin-paclitaxel and bevacizumab [143]. In patients with advanced solid tumors, another phase I dose-escalation study indicated that combination of pictilisib with EGFR tyrosine kinase inhibitor erlotinib was feasible [144]. In this study, modest antitumor effects were observed, that 2 (3.5\%) of 57 patients experienced PR and 19 (33.3\%) had stable disease [144]. A phase Ib dose-escalation study in patients with advanced non-small cell lung cancer assessed the tolerability and pharmacokinetics of pictilisib in combination with eitherpaclitaxel and carboplatin or pemetrexed and cisplatin, with or without bevacizumab [145]. In this study, pictilisib combination with various treatment regimens demonstrated promising efficacy and manageable toxicity, and preliminary antitumor activity was observed [145].

In a randomized, double-blind, placebo-controlled phase II study (FERGI) of oestrogen receptor-positive, aromatase inhibitor resistant advanced breast cancer, patients were randomly allocated (1:1 in part 1 and 2:1 in part 2) to pictilisib (340 mg daily in part 1 and $260 \mathrm{mg}$ daily in part 2) or placebo, plus intramuscular fulvestrant $500 \mathrm{mg}$. As a result, the addition of pictilisib to fulvestrant did not significantly improve PFS; it may be that the dose of pictilisib was limited by toxicity, potentially limiting its efficacy [146]. A phase II randomized PEGGY study in patients with hormone receptor-positive, HER2-negative, locally recurrent, or metastatic breast cancer revealed that adding pictilisib to paclitaxel did not prolong PFS of the patients [147]. In a randomized phase II study, patients with newly diagnosed estrogen receptor-positive, HER2 negative breast cancers were randomized to anastrozole or pictilisib plus anastrozole group [148]. The antitumor effects were measured by change of Ki-67 protein expression between tumor legions taken before and at the end of treatment [148]. Patients receiving the combination therapy showed greater geometric mean Ki-67 suppression from 66.0 to $83.8 \%$. Further, significant Ki-67 response was observed for patients with luminal B tumor, but not for patients with luminal A tumor [148].

\section{GDC-0032 (Taselisib)}

GDC-0032 (taselisib) is a potent and selective inhibitor of p110 $\alpha, \mathrm{p} 110 \delta$, and p110 $\gamma$ isoforms of class IA PI3K, with 31 folds less potency for the p110b isoform. Taselisib was progressed to clinical trials as a potential treatment for human cancer. A phase I study in Japanese patients showed that taselisib was well tolerated at $6 \mathrm{mg}$ daily in patients with advanced solid tumor, and $4 \mathrm{mg}$ daily in combination with fulvestrant in patients with HR-positive, HER2-negative advanced/recurrent breast cancer [149]. The most frequent treatment-related AEs were rash, diarrhea, and stomatitis. PR were observed in $2 / 9$ patients 
receiving monotherapy, and in 1/6 patients receiving combination therapy [149]. All patients with PR had PIK3CA-mutated tumor, which suggested that taselisib is expected to be effective in patients with PIK3CA-mutated solid tumor [149]. In another phase I dose escalation study of taselisib, 34 patients with locally advanced or metastatic solid tumor were given 3-16 mg taselisib once daily [150]. Dose limiting toxicities (DLT) were observed in patients receiving 12 and $16 \mathrm{mg}$ dose levels. Pharmacodynamic findings of patient tumor sample showed that PI3K pathway was inhibited at dose $\geq 3 \mathrm{mg} / \mathrm{d}$. Confirmed response was observed in 5/14 of PIK3CA-mutant tumor patients, and in 0/15 patients with tumors without known PIK3CA mutations [150]. A randomized phase III study of taselisib plus fulvestrant versus placebo plus fulvestrant in patients with metastatic breast cancer is ongoing.

\section{Isoform-specific inhibitors BYL719 (Alpelisib)}

BYL719 (alpelisib), an oral selective PI3K $\alpha$ isoform inhibitor, exhibited dose-dependent antitumor activity in tumor xenograft models, particularly models with mutated or amplified PIK3CA, highlighting the potential antitumor activity of alpelisib in patients with PIK3CA-altered tumors $[151,152]$. The first-in-human phase Ia study of alpelisib, demonstrated a tolerable safety profile and declared its MTD as $400 \mathrm{mg}$ daily and $150 \mathrm{mg}$ twice daily [153]. The most frequent treatment-related AEs included hyperglycemia, nausea, decreased appetite, diarrhea, and vomiting [153]. Among 134 patients with PIK3CA-altered advanced solid tumor who received treatment, stable disease was achieved in $70(52.2 \%)$ patients, PR was achieved in $7(5.2 \%)$ patients, and CR was achieved in $1(0.7 \%)$ patient [153]. In patients with ER-positive, HER2-negative metastatic breast cancer refractory to endocrine therapy, MTD of alpelisib in combination with letrozole was 300 $\mathrm{mg} / \mathrm{d}$ [154]. In this phase Ib study, the clinical antitumor activity was observed in 44\% patients with PIK3CA mutated and 20\% in PIK3CA wild-type tumors [154]. In trastuzumab- and taxane-resistant HER2-positive metastatic breast cancer, the combination of alpelisib and trastuzumab emtansine was tolerable and activity was observed, therefore further studies of the combination are expected to perform [155]. The triple-combination therapy of encorafenib (RAF kinase inhibitor), cetuximab (monoclonal antibody targeting EGFR) and alpelisib demonstrated promising clinical activity and tolerability in metastatic BRAF-mutant colorectal cancer patients [156]. A phase III study of alpelisib and fulvestrant is ongoing.

\section{CAL-101 (GS-1101, Idelalisib)}

CAL-101 (GS-1101, idelalisib) is an oral and specific inhibitor of the $\delta$ isoform of PI3K [122, 123]. It has been shown that idelalisib has therapeutic effects without inhibiting PI3K signaling essential for normal function of healthy cells $[157,158]$. Idelalisib is the frst FDA-approved PI3K inhibitor for use in combination with rituximab for the treatment of relapsed or refractory chronic lymphocytic leukemia, or as monotherapy for relapsed small lymphocytic lymphoma and follicular lymphoma previously treated with two or more prior systemic therapies.

In a phase Ib dose-escalation and extension studies of idelalisib, 64 patients with relapsed/refractory B-cell malignancies were assigned to one of eight regimens; idelalisib was taken once or twice a day at doses ranging from 50 to $350 \mathrm{mg}$ [159]. The ORR was $47 \%$ (30/64), with 1 patient had a CR $(1.6 \%)$. The median duration of response was 18.4 months, and the PFS was 7.6 months [159]. AEs were reported in $20 \%$ or more patients, including diarrhea, fatigue, nausea, and rash [159]. In this 48-week phase I clinical trial, the results of 40 patients with relapsed/refractory mantle cell lymphoma were reported in another article. Among this population, it was reported that the ORR was $40 \%(16 / 40)$, with CR in 5\% (2/40) patients. The median duration of response was 2.7 months, and the median PFS was 3.7 month [160]. In patients with relapsed/ refractory chronic lymphocytic leukemia, acceptable safety profile and antitumor activity of idelalisib were also reported [161]. A phase II trial in patients with chronic lymphocytic leukemia found that idelalisib used as upfront therapy caused an early, severe hepatotoxicity, particularly in younger subjects who have not received prior disease-specific therapy [162]. A single-group, open-label, phase II trial evaluating patients with relapsed (after receipt of rituximab and an alkylating agent) indolent non-Hodgkin lymphomas demonstrated similar findings; 125 patients were administered idelalisib $150 \mathrm{mg}$ twice daily [157]. The ORR was 57\% (71/125), and 6\% (7/125) met the criteria for CR, leading to FDA approval $[9,157$, 163]. The median duration of response was 12.5 months, and the median PFS was 11 months [157]. Moreover, in patients with relapsed/refractory classical Hodgkin lymphoma, idelalisib was tolerable and had modest single-agent activity, with an ORR of 20\% (5/25) [164].

The safety and efficacy of combined therapy with idelalisib and rituximab was evaluated in several clinical trials. In a phase II study of idelalisib plus rituximab, 64 treatment-naive older patients with chronic lymphocytic leukemia received rituximab $375 \mathrm{mg} / \mathrm{m}^{2}$ weekly and idelalisib $150 \mathrm{mg}$ twice daily; the ORR was $97 \%(62 / 64)$, including 19\% (12/64) CR [165]. Notably, the ORR was $100 \%$ in patients with $\operatorname{del}(17 \mathrm{p}) / \mathrm{TP} 53$ mutations. As compared with placebo and rituximab, this combined treatment significantly improved ORR ( $81 \%$ vs. $13 \%$; OR, 29.92; $P<0.001)$, PFS (HR, 0.15; $\mathrm{P}<0.001)$, and $\mathrm{OS}$ at 12 months $(92 \%$ vs. $80 \%$; HR, $0.28 ; P=0.02)$ among chronic lymphocytic leukemia patients who are less able to undergo standard chemotherapy [166]. However, the 
combination of idelalisib, lenalidomide and rituximab were not recommended for that excessively toxicity of this triplet regimen was reported in patients with relapsed and refractory lymphoma in a phase I trial [163]. In a global, randomised, phase III trial, idelalisib plus atumumab (a second-generation anti-CD20 antibody) resulted in better PFS (16.3 months vs.8.0 months, HR 0.27, $p<0.0001$ ) compared with atumumab alone in patients with relapsed chronic lymphocytic leukaemia progressing less than 24 months from the last therapy [167].

\section{Resistances}

The complexity of the PI3K/AKT/mTOR signaling network involves numerous feedback loops, extensive crosstalk nodes with other signaling pathways and compensatory pathways, providing ample opportunities for circumventing the effects of PI3K inhibition. Although small-molecule inhibitors of PI3K have exhibited promising clinical efficacy against human cancers, intrinsic and acquired resistance limits their therapeutic efficacy. Therefore, elucidating the mechanisms underlying resistance to $\mathrm{PI} 3 \mathrm{~K}$ inhibitor can provide rationale for combination therapies and alternative therapies. The specific mechanism is not completely defined; however, recent studies have described several possible resistance mechanisms, including PI3K reactivation, activation of parallel pathway, and tumor microenvironment.

Acquired amplification and mutation of PIK3CA and PIK3CB, which resulted in a marked upregulation of the PI3K signaling itself, have been shown to cause resistance to selective PI3K inhibitors $[168,169]$. As suggested previously, in the absence of PTEN, proliferation of cancer cells became dependent mostly on the activity of the $\mathrm{p} 110 \beta$ isoform $[170,171]$. The impact of PTEN loss on PI3K $\alpha$ inhibitor resistance has been proposed [172]. The loss of PTEN alone was not able to induce resistance to inhibitor of class I PI3K (GDC-0941), however, amphiregulin enhanced the resistance, which resulted in increased EGFR/ MAPK signaling. As a PI3K regulatory subunit, the phosphorylation of p85 has also been suggested to play a role in the development of resistance to PI3K inhibitors; presence of a regulatory loop between PI3K p85 and Src has also suggested contributing to resistance against PI3K inhibitors [173]. Intrinsic resistance to PI3K p110a Inhibitors was correlated with sustained mTORC1 activity; growth factors such as insulin-like growth factor 1 and neuregulin 1 can activate mTOR and thus mediate resistance to p110a inhibitors [152].

The RAS-RAF-MEK-ERK signaling pathway is highly interconnected with PI3K signaling [174]. Mutation and overexpression of HRAS which belongs to the RAS family has been shown to reduced susceptibility to PI3K inhibitor, while knockdown improved sensitivity [175]. Further, interactions between NEK9 and MAP2K4 have been proposed to mediate cancer cell proliferation and resistance to PI3K inhibitors [176]. PI3K inhibition with the pan-PI3K inhibitor GDC0941/ XL-147 or the dual PI3K/mTOR inhibitor BEZ235 has been shown to induce increased HER2/3 expression and lead to compensatory activation of the ERK signaling pathway $[177,178]$. Activation of STAT5 and expression of Pim kinases through STAT5 also conferred resistance to PI3K/AKT inhibitors by enhancing the mTORC1/Mcl-1 pathway [179]. Dual inhibition of PI3K and $\mathrm{m}$-TOR has been found to elicit a positive feedback response and lead to increases activation of JAK2/STAT5 and secretion of IL-8, thus contributing to drug resistance [180]. Moreover, IL6-STAT3 loop triggered epithelial-mesenchymal transition and expanded action cancer stem cells population, which have been proposed as one of the mechanisms [181]. Aberrant regulation of $\mathrm{WNT} / \beta$-catenin signaling and activation of GSK3 $\beta$ were correlated with resistance to the dual PI3K/mTOR inhibitor; nuclear $\beta$-catenin conferred resistance to the FOXO3a-mediated apoptosis provoked by PI3K and AKT inhibitors [182, 183].

Dual PI3K/mTOR inhibition led to activation of the NOTCH-MYC pathway [184]. NOTCH pathway and downstream induction of c-MYC were conferred resistance to PI3K inhibitors, whereas overexpression of the NOTCH canonical target genes HES1, HEY1 or HEY2 were not correlated with PI3K pathway inhibitor resistance $[37,184,185]$. The MYC was involved in growth, proliferation, differentiation, and metabolism of malignant cells, and knockdown of MYC reversed the resistance to dual PI3K/mTOR inhibitor [184]. Previous studies have also indicated that amplification of both MYC and eIF4E can mediate resistance to PI3K/m-TOR inhibitors [186]. eIF4E is an established MYC regulated target, indicate that interactions between MYC and eIF4E in regulating resistance mechanism is a possibility [184].

Proviral Integration site for Moloney murine leukemic virus (PIM) which overexpress in multiple malignancies has been shown to confer resistance by maintaining activation of downstream PI3K effectors in an AKT-independent manner [187]. In addition, PIM has been reported to modulate the activity of eIF4B and mTORC1 to enhance NRF2/ARE activity, and to decrease ROS production to diminish the cytotoxicity of PI3K/AKT inhibitors [188]. S-phase kinase-associated protein 2 (Skp2) could promote the activation of AKT, and it has been reported to correlate with the resistance of PI3K inhibition [189]. Amplification or overexpression of RSK3 (Ribosomal S6 kinases RPS6KA2), RSK4 ((Ribosomal S6 kinases RPS6KA4), PAK1, CDK 4/6, MSK1 (mitogen- and stress-activated protein kinase 1), KDM6B, and IGFBP5 have also been shown to confer resistance to PI3K inhibitors [168, 190-192].

High amounts of purine-related aqueous metabolites like hypoxanthine, and high levels of the mRNA encoding hypoxanthine phosphoribosyl transferase 1 (one of the key components of the purine salvage pathway), have 
been found to be associated with resistance of PI3K pathway inhibition [193]. In consideration of the fact that ncRNA have been reported to regulate PI3K signaling and other parallel pathways (e.g. WNT/ $\beta$-catenin, RAS/ERK/ MAPK, JAK/STAT, NOTCH), we believe ncRNA may also play a role in the resistance of PI3K inhibitors [194-199]. Not surprisingly, more and more researches have suggested that deviant ncRNA expression is powerfully concerned about tumor drug resistance [200-208]. Recent studies have indicated potential mechanism of acquired resistance to dual PI3K/mTOR inhibitors, including elevated glycolysis accompanied with depletion of mitochondrial DNA, and upregulated DNA methyltransferases which Reduce PTEN and PPP2R2B expression [209, 210]. Novel roles of the tumor microenvironment have introduced in regulating drug resistance, and macrophages in microenvironment have been proposed as factors contributing to the resistances of PI3K inhibitors through the activation of NF- $\mathrm{kB}$ signaling [211].

\section{Conclusions}

The PI3K signaling pathway plays an important role in cell growth, proliferation and survival, making PI3K inhibition an attractive target for anticancer therapy. However, clinical trials with PI3K inhibitors used as a monotherapy have shown limited clinical activity, possibly as a consequence of resistance to PI3K inhibition and poor tolerability of PI3K inhibitors. Dual PI3K/ mTOR and pan-PI3K inhibitors have made their way into clinical trials with limited efficacy as monotherapy, and relatively high rates of side effects were reported. As it has been increasingly recognized that different isoforms of PI3K play non-redundant roles in particular tumor types, isoform-selective inhibitors were developed. Isoform-selective PI3K inhibitors demonstrate improved specificity and reduced toxicity over dual PI3K/ mTOR and pan-PI3K inhibitors, which have shown promising success in several clinical trials for both solid and hematological malignancies.

Several studies showed that PI3K inhibitors were more effective in patients with PI3K pathway mutations, however, some patients without documented PI3K mutations benefited from PI3K inhibitors and some patients with PIK3CA or other mutations not experienced benefit. As a result, strong correlations between PI3K mutations and response to therapy still have not been established in preclinical and clinical studies. It is important to identify reliable biomarkers that can guide patient selection, and to determine which tumor type and genetic profiles will benefit from PI3K inhibition. It is reported the value of pharmacodynamic biomarkers and functional imaging monitoring biomarker in guiding the selection of patients who are most likely to respond to PI3K inhibition, but the precision is still controversial [212]. To date, the mechanism of PI3K inhibitors has not been well established. The precise mechanism needs to be extensively and systematically studied, so that it will allow us to monitor efficacy and side effects, and to make personalized therapeutic decisions.

Preliminary clinical data indicated that the use of single-agent PI3K pathway inhibitors achieved modest responses and was unlikely to be a curative therapy for diverse cancers. The efficacy of PI3K inhibitors is limited for their narrow therapeutic window and frequent treatment-related toxicities. The drugs recommended are more likely to be optimally used in combination with other therapeutic modalities, such as surgery, hormonal therapies and other anticancer agents. Introduction of tumor suppressive or knockdown of oncogenic ncRNAs would be a feasible approach to inhibit the PI3K pathway. The combination of PI3K inhibitors with ncRNAs or inhibitors against other cross-talk pathways might yield promising therapeutic effects. AEs, including nausea, vomiting, diarrhea, hyperglycemia, fatigue, rash, anorexia, and abnormal hepatic function were frequently reported. These combination strategies may also decrease the rates of AEs and minimize the risk of the development of resistance.

Overall, PI3K inhibition is being investigated as a potential strategy to develop novel therapeutics for cancer management. Although we move forward with the clinical development of PI3K inhibitors, maximizing the utility of these agents in the treatment of patients remains challenging. Certainly, understanding the precise mechanisms of PI3K signaling and PI3K inhibition will be critical. Optimization of the patient selection strategies and combination approaches will help increase the practical efficacy of these agents. Continued work to clarify the resistance mechanisms and the novel strategies to overcome resistance will also be important.

\section{Abbreviations \\ AEs: Adverse events; CR: Complete responses; ctDNA: Circulating tumor DNA; FDA: Food and Drug Administration; HR: Hazard ratio; LncRNA: Long non-coding RNA; miRNA: MicroRNA; MTD: Maximum tolerated dose; mTOR: Mammalian target of rapamycin; ncRNA: Non-coding RNA; ORR: Overall response rates; OS: Overall survival; PFS: Progression-free survival; PI3K: Phosphatidylinositol-3-kinase; PIP2: Phosphorylation of Ptdlns(4,5) P2; PIP3: Ptdlns(3,4,5) P3; PR: Partial responses; PTEN: Phosphatase and tensin homologue deleted on chromosome 10; RDE: Recommended dose for expansion}

\section{Acknowledgements}

We express our gratitude to Mr. Lewis for his writing assistance.

\section{Funding}

This work is supported by the National Natural Science Foundation of China (No. 81602492), the National Key Research and Development Program of China (No. 2016YFA0201402) and by the National Major Scientific and Technological Special Project for "Significant New Drugs Development" (No. 2018ZX09733001).

Availability of data and materials Not applicable. 


\section{Authors' contributions}

JY Data curation; Formal analysis; Writing - original draft. JN Data curation; Formal analysis; Writing - original draft. XM Writing - review \& editing. YW Conceptualization; Supervision. All authors read and approved the final manuscript. YP Conceptualization; Supervision. XW Conceptualization; Supervision; Validation; Writing - review editing.

\section{Authors' information}

Not applicable.

\section{Ethics approval and consent to participate}

Not applicable.

\section{Consent for publication}

Not applicable.

\section{Competing interests}

The authors declare that they have no competing interests.

\section{Publisher's Note}

Springer Nature remains neutral with regard to jurisdictional claims in published maps and institutional affiliations.

Received: 2 January 2019 Accepted: 6 February 2019 Published online: 19 February 2019

\section{References}

1. Katso R, Okkenhaug K, Ahmadi K, White S, Timms J, Waterfield MD. Cellular function of phosphoinositide 3-kinases: implications for development, homeostasis, and cancer. Annu Rev Cell Dev Biol. 2001;17:615-75.

2. Engelman JA, Luo J, Cantley LC. The evolution of phosphatidylinositol 3kinases as regulators of growth and metabolism. Nat Rev Genet. 2006;7: 606-19.

3. Martini M, De Santis MC, Braccini L, Gulluni F, Hirsch E. PI3K AKT signaling pathway and cancer: an updated review. Ann Med. 2014;46:372-83.

4. Klingenberg M, Matsuda A, Diederichs S, Patel T. Non-coding RNA in hepatocellular carcinoma: Mechanisms, biomarkers and therapeutic targets. J Hepatol. 2017;67:603-18.

5. Benetatos L, Voulgaris $\mathrm{E}$, Vartholomatos $\mathrm{G}$. The crosstalk between long noncoding RNAs and PI3K in cancer. Med Oncol. 2017;34:39.

6. Dong P, Konno Y, Watari H, Hosaka M, Noguchi M, Sakuragi N. The impact of microRNA-mediated PI3K/AKT signaling on epithelial-mesenchymal transition and cancer stemness in endometrial cancer. J Transl Med. 2014; 12:231.

7. Asati V, Mahapatra DK, Bharti SK. PI3K/Akt/mTOR and Ras/Raf/MEK/ERK signaling pathways inhibitors as anticancer agents: Structural and pharmacological perspectives. Eur J Med Chem. 2016;109:314-41.

8. Hennessy BT, Smith DL, Ram PT, Lu Y, Mills GB. Exploiting the PI3K/AKT pathway for cancer drug discovery. Nat Rev Drug Discov. 2005;4:988-1004.

9. Markham A. Idelalisib: first global approval. Drugs. 2014;74:1701-7.

10. Donahue TR, Tran LM, Hill R, Li Y, Kovochich A, Calvopina JH, Patel SG, Wu $\mathrm{N}$, Hindoyan A, Farrell JJ, et al. Integrative survival-based molecular profiling of human pancreatic cancer. Clin Cancer Res. 2012;18:1352-63.

11. Yuan TL, Cantley LC. PI3K pathway alterations in cancer: variations on a theme. Oncogene. 2008;27:5497-510.

12. Pacold ME, Suire $S$, Perisic $O$, Lara-Gonzalez S, Davis $C T$, Walker EH, Hawkins PT, Stephens L, Eccleston JF, Williams RL. Crystal structure and functional analysis of Ras binding to its effector phosphoinositide 3-kinase gamma. Cell. 2000;103:931-43.

13. Guo H, German P, Bai S, Barnes S, Guo W, Qi X, Lou H, Liang J, Jonasch E, Mills GB, Ding Z. The PI3K/AKT Pathway and Renal Cell Carcinoma. J Genet Genomics. 2015;42:343-53.

14. Manning BD, Cantley LC. AKT/PKB signaling: navigating downstream. Cell. 2007;129:1261-74

15. Dillon RL, White DE, Muller WJ. The phosphatidyl inositol 3-kinase signaling network: implications for human breast cancer. Oncogene. 2007;26:1338-45.

16. Aziz SA, Davies M, Pick E, Zito C, Jilaveanu L, Camp RL, Rimm DL, Kluger Y, Kluger HM. Phosphatidylinositol-3-kinase as a therapeutic target in melanoma. Clin Cancer Res. 2009;15:3029-36.

17. Stemke-Hale K, Gonzalez-Angulo AM, Lluch A, Neve RM, Kuo WL, Davies M, Carey M, Hu Z, Guan Y, Sahin A, et al. An integrative genomic and proteomic analysis of PIK3CA, PTEN, and AKT mutations in breast cancer Cancer Res. 2008:68:6084-91.

18. Zhou BP, Hu MC, Miller SA, Yu Z, Xia W, Lin SY, Hung MC. HER-2/neu blocks tumor necrosis factor-induced apoptosis via the Akt/NF-kappaB pathway. J Biol Chem. 2000;275:8027-31.

19. Stambolic V, Suzuki A, de la Pompa JL, Brothers GM, Mirtsos C, Sasaki T, Ruland J, Penninger JM, Siderovski DP, Mak TW. Negative regulation of PKB/Aktdependent cell survival by the tumor suppressor PTEN. Cell. 1998;95:29-39.

20. Papa A, Wan L, Bonora M, Salmena L, Song MS, Hobbs RM, Lunardi A, Webster K, Ng C, Newton RH, et al. Cancer-associated PTEN mutants act in a dominantnegative manner to suppress PTEN protein function. Cell. 2014;157:595-610.

21. Li J, Yen C, Liaw D, Podsypanina K, Bose S, Wang SI, Puc J, Miliaresis C, Rodgers L, McCombie R, et al. PTEN, a putative protein tyrosine phosphatase gene mutated in human brain, breast, and prostate cancer. Science. 1997;275:1943-7.

22. Liaw D, Marsh DJ, Li J, Dahia PL, Wang SI, Zheng Z, Bose S, Call KM, Tsou HC, Peacocke M, et al. Germline mutations of the PTEN gene in Cowden disease, an inherited breast and thyroid cancer syndrome. Nat Genet. 1997;16:64-7.

23. Chen CY, Chen J, He L, Stiles BL. PTEN: Tumor Suppressor and Metabolic Regulator. Front Endocrinol (Lausanne). 2018;9:338.

24. Carracedo A, Alimonti A, Pandolfi PP. PTEN level in tumor suppression: how much is too little? Cancer Res. 2011;71:629-33.

25. Haddadi N, Lin Y, Travis G, Simpson AM, Nassif NT, McGowan EM. PTEN/ PTENP1: 'Regulating the regulator of RTK-dependent PI3K/Akt signalling', new targets for cancer therapy. Mol Cancer. 2018;17:37.

26. Samuels Y, Wang Z, Bardelli A, Silliman N, Ptak J, Szabo S, Yan H, Gazdar A, Powell SM, Riggins GJ, et al. High frequency of mutations of the PIK3CA gene in human cancers. Science. 2004;304:554.

27. Levine DA, Bogomolniy F, Yee CJ, Lash A, Barakat RR, Borgen PI, Boyd J. Frequent mutation of the PIK3CA gene in ovarian and breast cancers. Clin Cancer Res. 2005;11:2875-8.

28. Lee JW, Soung YH, Kim SY, Lee HW, Park WS, Nam SW, Kim SH, Lee JY, Yoo $\mathrm{NJ}$, Lee SH. PIK3CA gene is frequently mutated in breast carcinomas and hepatocellular carcinomas. Oncogene. 2005;24:1477-80.

29. Ma YY, Wei SJ, Lin YC, Lung JC, Chang TC, Whang-Peng J, Liu JM, Yang DM, Yang WK, Shen CY. PIK3CA as an oncogene in cervical cancer. Oncogene. 2000;19:2739-44.

30. Xiang $L$, Jiang $W$, Li J, Shen $X$, Yang W, Yang G, Wu X, Yang H. PIK3CA mutation analysis in Chinese patients with surgically resected cervical cancer. Sci Rep. 2015:5:14035.

31. Triscott J, Rubin MA. Prostate Power Play: Does Pik3ca Accelerate PtenDeficient Cancer Progression? Cancer Discov. 2018;8:682-5.

32. Ikenoue T, Kanai F, Hikiba Y, Obata T, Tanaka Y, Imamura J, Ohta M, Jazag A, Guleng B, Tateishi $K$, et al. Functional analysis of PIK3CA gene mutations in human colorectal cancer. Cancer Res. 2005;65:4562-7.

33. Jiang $W$, He T, Liu S, Zheng Y, Xiang L, Pei X, Wang Z, Yang H. The PIK3CA E542K and E545K mutations promote glycolysis and proliferation via induction of the beta-catenin/SIRT3 signaling pathway in cervical cancer. J Hematol Oncol. 2018;11:139.

34. Kang S, Bader AG, Vogt PK. Phosphatidylinositol 3-kinase mutations identified in human cancer are oncogenic. Proc Natl Acad Sci U S A. 2005;102:802-7.

35. Zhao L, Vogt PK. Helical domain and kinase domain mutations in p110alpha of phosphatidylinositol 3-kinase induce gain of function by different mechanisms. Proc Natl Acad Sci U S A. 2008;105:2652-7.

36. Croessmann S, Sheehan JH, Lee KM, Sliwoski G, He J, Nagy R, Riddle D, Mayer IA, Balko JM, Lanman R, et al. PIK3CA C2 Domain Deletions Hyperactivate Phosphoinositide 3-kinase (PI3K), Generate Oncogene Dependence, and Are Exquisitely Sensitive to PI3Kalpha Inhibitors. Clin Cancer Res. 2018:24:1426-35.

37. Liu P, Cheng H, Santiago S, Raeder M, Zhang F, Isabella A, Yang J, Semaan DJ, Chen C, Fox EA, et al. Oncogenic PIK3CA-driven mammary tumors frequently recur via PI3K pathway-dependent and PI3K pathwayindependent mechanisms. Nat Med. 2011;17:1116-20.

38. Yuan W, Stawiski E, Janakiraman V, Chan E, Durinck S, Edgar KA, Kljavin NM, Rivers CS, Gnad F, Roose-Girma M, et al. Conditional activation of $\mathrm{Pik3ca}(\mathrm{H} 1047 \mathrm{R})$ in a knock-in mouse model promotes mammary tumorigenesis and emergence of mutations. Oncogene. 2013;32:318-26.

39. Kinross KM, Montgomery KG, Kleinschmidt M, Waring P, Ivetac I, Tikoo A, Saad M, Hare L, Roh V, Mantamadiotis T, et al. An activating Pik3ca mutation coupled with Pten loss is sufficient to initiate ovarian tumorigenesis in mice. J Clin Invest. 2012;122:553-7. 
40. Aoki M, Schetter C, Himly M, Batista O, Chang HW, Vogt PK. The catalytic subunit of phosphoinositide 3-kinase: requirements for oncogenicity. J Biol Chem. 2000;275:6267-75.

41. Czauderna F, Fechtner M, Aygun H, Arnold W, Klippel A, Giese K, Kaufmann J. Functional studies of the PI(3)-kinase signalling pathway employing synthetic and expressed siRNA. Nucleic Acids Res. 2003;31:670-82.

42. Hill KM, Kalifa S, Das JR, Bhatti T, Gay M, Williams D, Taliferro-Smith L, De Marzo AM. The role of PI 3-kinase p110beta in AKT signally, cell survival, and proliferation in human prostate cancer cells. Prostate. 2010;70:755-64.

43. Dbouk HA, Khalil BD, Wu H, Shymanets A, Nurnberg B, Backer JM. Characterization of a tumor-associated activating mutation of the p110beta PI 3-kinase. PLoS One. 2013;8:e63833.

44. Dbouk HA, Vadas O, Shymanets A, Burke JE, Salamon RS, Khalil BD, Barrett MO, Waldo GL, Surve C, Hsueh C, et al. G protein-coupled receptormediated activation of p110beta by Gbetagamma is required for cellular transformation and invasiveness. Sci Signal. 2012;5:ra89.

45. Kan Z, Jaiswal BS, Stinson J, Janakiraman V, Bhatt D, Stern HM, Yue P, Haverty PM, Bourgon R, Zheng J, et al. Diverse somatic mutation patterns and pathway alterations in human cancers. Nature. 2010;466:869-73.

46. Pazarentzos E, Giannikopoulos P, Hrustanovic G, St John J, Olivas VR, Gubens MA, Balassanian R, Weissman J, Polkinghorn W, Bivona TG. Oncogenic activation of the PI3-kinase p110beta isoform via the tumor-derived PIK3Cbeta(D1067V) kinase domain mutation. Oncogene. 2016;35:1198-205.

47. Kim E, llic N, Shrestha Y, Zou L, Kamburov A, Zhu C, Yang X, Lubonja R, Tran N, Nguyen C, et al. Systematic Functional Interrogation of Rare Cancer Variants Identifies Oncogenic Alleles. Cancer Discov. 2016;6:714-26.

48. Fung-Leung WP. Phosphoinositide 3-kinase delta (PI3Kdelta) in leukocyte signaling and function. Cell Signal. 2011;23:603-8.

49. Okkenhaug K. Signaling by the phosphoinositide 3-kinase family in immune cells. Annu Rev Immunol. 2013;31:675-704.

50. Compagno M, Wang Q, Pighi C, Cheong TC, Meng FL, Poggio T, Yeap LS, Karaca E, Blasco RB, Langellotto F, et al. Phosphatidylinositol 3-kinase delta blockade increases genomic instability in B cells. Nature. 2017;542:489-93.

51. Lucas CL, Chandra A, Nejentsev S, Condliffe AM, Okkenhaug K. PI3Kdelta and primary immunodeficiencies. Nat Rev Immunol. 2016;16:702-14.

52. Sujobert $P$, Bardet V, Cornillet-Lefebvre $P$, Hayflick JS, Prie N, Verdier F, Vanhaesebroeck B, Muller O, Pesce F, Ifrah N, et al. Essential role for the p110delta isoform in phosphoinositide 3-kinase activation and cell proliferation in acute myeloid leukemia. Blood. 2005;106:1063-6.

53. Zhang J, Grubor V, Love CL, Banerjee A, Richards KL, Mieczkowski PA, Dunphy C, Choi W, Au WY, Srivastava G, et al. Genetic heterogeneity of diffuse large B-cell lymphoma. Proc Natl Acad Sci U S A. 2013;110:1398-403.

54. Sawyer C, Sturge J, Bennett DC, O'Hare MJ, Allen WE, Bain J, Jones GE, Vanhaesebroeck B. Regulation of breast cancer cell chemotaxis by the phosphoinositide 3-kinase p110delta. Cancer Res. 2003;63:1667-75.

55. Kaneda MM, Messer KS, Ralainirina N, Li H, Leem CJ, Gorjestani S, Woo G, Nguyen AV, Figueiredo CC, Foubert P, et al. PI3Kgamma is a molecular switch that controls immune suppression. Nature. 2016;539:437-42.

56. Hawkins PT, Stephens LR. PI3K signalling in inflammation. Biochim Biophys Acta. 1851;2015:882-97.

57. Stockmann C, Doedens A, Weidemann A, Zhang N, Takeda N, Greenberg Jl, Cheresh DA, Johnson RS. Deletion of vascular endothelial growth factor in myeloid cells accelerates tumorigenesis. Nature. 2008:456:814-8.

58. Bauer TM, Patel MR, Infante JR. Targeting PI3 kinase in cancer. Pharmacol Ther. 2015;146:53-60.

59. Andreopoulou E. The PI3K/AKT/mTOR signaling pathway: Implications in the treatment of breast cancer. Current Breast Cancer Reports. 2011;3:63-74.

60. Shen S, Liu H, Wang Y, Wang J, Ni X, Ai Z, Pan H, Liu H, Shao Y. Long noncoding RNA CRNDE promotes gallbladder carcinoma carcinogenesis and as a scaffold of DMBT1 and C-IAP1 complexes to activating PI3K-AKT pathway. Oncotarget. 2016;7:72833-44

61. Liu XX, Xiong HP, Huang JS, Qi K, Xu JJ. Highly expressed long noncoding RNA CRNDE promotes cell proliferation through PI3K/AKT signalling in non-small cell lung carcinoma. Clin Exp Pharmacol Physiol. 2017;44:895-902.

62. Ellis BC, Graham LD, Molloy PL. CRNDE, a long non-coding RNA responsive to insulin/IGF signaling, regulates genes involved in central metabolism. Biochim Biophys Acta. 1843;2014:372-86.

63. Du DX, Lian DB, Amin BH, Yan W. Long non-coding RNA CRNDE is a novel tumor promoter by modulating PI3K/AKT signal pathways in human gastric cancer. Eur Rev Med Pharmacol Sci. 2017;21:5392-98.
64. Yang HY, Huang CP, Cao MM, Wang YF, Liu Y. Long non-coding RNA CRNDE may be associated with poor prognosis by promoting proliferation and inhibiting apoptosis of cervical cancer cells through targeting PI3K/AKT. Neoplasma. 2018;65:872-80.

65. Tang Q, Zheng X, Zhang J. Long non-coding RNA CRNDE promotes heptaocellular carcinoma cell proliferation by regulating PI3K/Akt /betacatenin signaling. Biomed Pharmacother. 2018;103:1187-93.

66. Wang Y, Kong D. LncRNA GAS5 Represses Osteosarcoma Cells Growth and Metastasis via Sponging MiR-203a. Cell Physiol Biochem. 2018;45:844-55.

67. Wang G, Sun J, Zhao H, Li H. Long Non-Coding RNA (IncRNA) Growth Arrest Specific 5 (GAS5) Suppresses Esophageal Squamous Cell Carcinoma Cell Proliferation and Migration by Inactivating Phosphatidylinositol 3-kinase (PI3K)/AKT/Mammalian Target of Rapamycin (mTOR) Signaling Pathway. Med Sci Monit. 2018;24:7689-96.

68. Li S, Zhou J, Wang Z, Wang P, Gao X, Wang Y. Long noncoding RNA GAS5 suppresses triple negative breast cancer progression through inhibition of proliferation and invasion by competitively binding miR-196a-5p. Biomed Pharmacother. 2018;104:451-7.

69. Yuan S, Wu Y, Wang Y, Chen J, Chu L. An Oncolytic Adenovirus Expressing SNORD44 and GAS5 Exhibits Antitumor Effect in Colorectal Cancer Cells. Hum Gene Ther. 2017;28:690-700.

70. Renganathan A, Kresoja-Rakic J, Echeverry N, Ziltener G, Vrugt B, Opitz I, Stahel RA, Felley-Bosco E. GAS5 long non-coding RNA in malignant pleural mesothelioma. Mol Cancer. 2014;13:119.

71. Pickard MR, Williams GT. Regulation of apoptosis by long non-coding RNA GAS5 in breast cancer cells: implications for chemotherapy. Breast Cancer Res Treat. 2014;145:359-70.

72. Yacqub-Usman K, Pickard MR, Williams GT. Reciprocal regulation of GAS5 InCRNA levels and mTOR inhibitor action in prostate cancer cells. Prostate. 2015:75:693-705.

73. Aziz SA, Jilaveanu LB, Zito C, Camp RL, Rimm DL, Conrad P, Kluger HM. Vertical targeting of the phosphatidylinositol-3 kinase pathway as a strategy for treating melanoma. Clin Cancer Res. 2010;16:6029-39.

74. Calero R, Morchon E, Martinez-Argudo I, Serrano R. Synergistic anti-tumor effect of 17AAG with the PI3K/mTOR inhibitor NVP-BEZ235 on human melanoma. Cancer Lett. 2017:406:1-11.

75. Marone R, Erhart D, Mertz AC, Bohnacker T, Schnell C, Cmiljanovic V, Stauffer F, Garcia-Echeverria C, Giese B, Maira SM, Wymann MP. Targeting melanoma with dual phosphoinositide 3-kinase/mammalian target of rapamycin inhibitors. Mol Cancer Res. 2009;7:601-13.

76. Wang Y, Yu Q, He X, Romigh T, Altemus J, Eng C. Activation of AR sensitizes breast carcinomas to NVP-BEZ235's therapeutic effect mediated by PTEN and KLLN upregulation. Mol Cancer Ther. 2014;13:517-27.

77. Brachmann SM, Hofmann I, Schnell C, Fritsch C, Wee S, Lane H, Wang S, Garcia-Echeverria C, Maira SM. Specific apoptosis induction by the dual PI3K/mTor inhibitor NVP-BEZ235 in HER2 amplified and PIK3CA mutant breast cancer cells. Proc Natl Acad Sci U S A. 2009;106:22299-304.

78. Martinelli E, Troiani T, D'Aiuto E, Morgillo F, Vitagliano D, Capasso A, Costantino S, Ciuffreda LP, Merolla F, Vecchione L, et al. Antitumor activity of pimasertib, a selective MEK $1 / 2$ inhibitor, in combination with PI3K/mTOR inhibitors or with multi-targeted kinase inhibitors in pimasertib-resistant human lung and colorectal cancer cells. Int J Cancer. 2013;133:2089-101.

79. Yang X, Niu B, Wang L, Chen M, Kang X, Wang L, Ji Y, Zhong J. Autophagy inhibition enhances colorectal cancer apoptosis induced by dual phosphatidylinositol 3-kinase/mammalian target of rapamycin inhibitor NVP-BEZ235. Oncol Lett. 2016;12:102-6.

80. Kudoh A, Oishi T, Itamochi H, Sato S, Naniwa J, Sato S, Shimada M, Kigawa J, Harada T. Dual inhibition of phosphatidylinositol 3'-kinase and mammalian target of rapamycin using NVP-BEZ235 as a novel therapeutic approach for mucinous adenocarcinoma of the ovary. Int J Gynecol Cancer. 2014;24:444-53.

81. Herrera VA, Zeindl-Eberhart E, Jung A, Huber RM, Bergner A. The dual PI3K mTOR inhibitor BEZ235 is effective in lung cancer cell lines. Anticancer Res. 2011:31:849-54.

82. Bhende PM, Park SI, Lim MS, Dittmer DP, Damania B. The dual PI3K/mTOR inhibitor, NVP-BEZ235, is efficacious against follicular lymphoma. Leukemia. 2010;24:1781-4

83. Cho DC, Cohen MB, Panka DJ, Collins M, Ghebremichael M, Atkins MB, Signoretti S, Mier JW. The efficacy of the novel dual PI3-kinase/mTOR inhibitor NVP-BEZ235 compared with rapamycin in renal cell carcinoma. Clin Cancer Res. 2010;16:3628-38. 
84. Zhu W, Fu W, Hu L. NVP-BEZ235, dual phosphatidylinositol 3-kinase/ mammalian target of rapamycin inhibitor, prominently enhances radiosensitivity of prostate cancer cell line PC-3. Cancer Biother Radiopharm. 2013;28:665-73.

85. Park HS, Hong SK, Oh MM, Yoon CY, Jeong SJ, Byun SS, Cheon J, Lee SE. Moon du G: Synergistic antitumor effect of NVP-BEZ235 and sunitinib on docetaxel-resistant human castration-resistant prostate cancer cells. Anticancer Res. 2014;34:3457-68.

86. Massard C, Chi KN, Castellano D, de Bono J, Gravis G, Dirix L, Machiels JP, Mita A, Mellado B, Turri S, et al. Phase Ib dose-finding study of abiraterone acetate plus buparlisib (BKM120) or dactolisib (BEZ235) in patients with castration-resistant prostate cancer. Eur J Cancer. 2017;76:36-44.

87. Wise-Draper TM, Moorthy G, Salkeni MA, Karim NA, Thomas HE, Mercer CA Beg MS, O'Gara S, Olowokure O, Fathallah H, et al. A Phase lb Study of the Dual PI3K/mTOR Inhibitor Dactolisib (BEZ235) Combined with Everolimus in Patients with Advanced Solid Malignancies. Target Oncol. 2017;12:323-32.

88. Fazio N, Buzzoni R, Baudin E, Antonuzzo L, Hubner RA, Lahner H, De Herder WW, Raderer M, Teule A, Capdevila J, et al. A Phase II Study of BEZ235 in Patients with Everolimus-resistant, Advanced Pancreatic Neuroendocrine Tumours. Anticancer Res. 2016;36:713-9.

89. Salazar R, Garcia-Carbonero R, Libutti SK, Hendifar AE, Custodio A, Guimbaud R, Lombard-Bohas C, Ricci S, Klumpen HJ, Capdevila J, et al. Phase II Study of BEZ235 versus Everolimus in Patients with Mammalian Target of Rapamycin Inhibitor-Naive Advanced Pancreatic Neuroendocrine Tumors. Oncologist. 2018;23:766-e790.

90. Dolly SO, Wagner AJ, Bendell JC, Kindler HL, Krug LM, Seiwert TY, Zauderer MG, Lolkema MP, Apt D, Yeh RF, et al. Phase I Study of Apitolisib (GDC-0980), Dual Phosphatidylinositol-3-Kinase and Mammalian Target of Rapamycin Kinase Inhibitor, in Patients with Advanced Solid Tumors. Clin Cancer Res. 2016;22:2874-84.

91. Rosen LS, Goldman J, Stewart S, Hubbard JM, Roos M, Lin W, Shankar G, Capdevila J, Freas E, Leong S. A phase $1 \mathrm{~b}$ study to evaluate the safety and pharmacology of the dual PI3K-mtor inhibitor GDC-0980 in combination with a fluoropyrimidine, oxaliplatin, and bevacizumab (BEV) in patients with advanced solid tumors. Ann Oncol. 2012;9:ix157.

92. Powles T, Lackner MR, Oudard S, Escudier B, Ralph C, Brown JE, Hawkins RE, Castellano D, Rini BI, Staehler MD, et al. Randomized Open-Label Phase II Trial of Apitolisib (GDC-0980), a Novel Inhibitor of the PI3K/Mammalian Target of Rapamycin Pathway, Versus Everolimus in Patients With Metastatic Renal Cell Carcinoma. J Clin Oncol. 2016:34:1660-8.

93. Powles T, Oudard S, Escudier BJ, Brown JE, Hawkins RE, Castellano DE, Ravaud A, Staehler MD, Rini BI, Lin W, et al. A randomized phase II study of GDC-0980 versus everolimus in metastatic renal cell carcinoma (mRCC) patients (pts) after VEGF-targeted therapy (VEGF-TT). Journal of Clinical Oncology 2014;32:4525-25.

94. Makker V, Recio FO, Ma L, Matulonis UA, Lauchle JO, Parmar H, Gilbert HN, Ware JA, Zhu R, Lu S, et al. A multicenter, single-arm, open-label, phase 2 study of apitolisib (GDC-0980) for the treatment of recurrent or persistent endometrial carcinoma (MAGGIE study). Cancer. 2016;122:3519-28.

95. Rosen L, Goldman J, Hubbard JM, Roos M, Capdevila J, Maynes J, Lin W, O'Keeffe B, Lackner M, Spoerke J, et al. Phase Ib study of oral dual-PI3K mTOR inhibitor GDC-0980 in combination with capecitabine and mFOLFOX6 + bevacizumab in patients with advanced solid tumors and colorectal cancer. Eur J Cancer. 2014:6:122-3.

96. Herzog A, Bian Y, Vander Broek R, Hall B, Coupar J, Cheng H, Sowers AL, Cook JD, Mitchell JB, Chen Z, et al. PI3K/mTOR inhibitor PF-04691502 antitumor activity is enhanced with induction of wild-type TP53 in human xenograft and murine knockout models of head and neck cancer. Clin Cancer Res. 2013;19:3808-19.

97. Yuan J, Mehta PP, Yin MJ, Sun S, Zou A, Chen J, Rafidi K, Feng Z, Nickel J, Engebretsen J, et al. PF-04691502, a potent and selective oral inhibitor of PI3K and mTOR kinases with antitumor activity. Mol Cancer Ther. 2011;10:2189-99.

98. Soares HP, Xu Q, Young SH, Sinnet-Smith J, Rozengurt E. Dual PI3K/mTOR inhibitors induce rapid over-activation of the MEK/ERK pathway in human pancreatic cancer cells through suppression of mTORC2. Pancreas. 2015;44(8):1416.

99. Freitag H, Christen F, Lewens F, Grass I, Briest F, Iwaszkiewicz S, Siegmund B, Grabowski P. Inhibition of mTOR's Catalytic Site by PKI-587 Is a Promising Therapeutic Option for Gastroenteropancreatic Neuroendocrine Tumor Disease. Neuroendocrinol. 2017;105:90-104

100. Shapiro Gl, Bell-McGuinn KM, Molina JR, Bendell J, Spicer J, Kwak EL, Pandya SS, Millham R, Borzillo G, Pierce KJ, et al. First-in-Human Study of PF05212384 (PKI-587), a Small-Molecule, Intravenous, Dual Inhibitor of PI3K and mTOR in Patients with Advanced Cancer. Clin Cancer Res. 2015;21: 1888-95.

101. Britten CD, Adjei AA, Millham R, Houk BE, Borzillo G, Pierce K, Wainberg ZA, LoRusso PM. Phase I study of PF-04691502, a small-molecule, oral, dual inhibitor of PI3K and mTOR, in patients with advanced cancer. Invest New Drugs. 2014;32:510-7.

102. Wainberg ZA, Alsina M, Soares HP, Brana I, Britten CD, Del Conte G, Ezeh P, Houk B, Kern KA, Leong S, et al. A Multi-Arm Phase I Study of the PI3K/mTOR Inhibitors PF-04691502 and Gedatolisib (PF-05212384) plus Irinotecan or the MEK Inhibitor PD-0325901 in Advanced Cancer. Target Oncol. 2017;12:775-85.

103. Del Campo JM, Birrer M, Davis C, Fujiwara K, Gollerkeri A, Gore M, Houk B, Lau S, Poveda A, Gonzalez-Martin A, et al. A randomized phase II noncomparative study of PF-04691502 and gedatolisib (PF-05212384) in patients with recurrent endometrial cancer. Gynecol Oncol. 2016;142:62-9.

104. Maira SM, Pecchi S, Huang A, Burger M, Knapp M, Sterker D, Schnell C, Guthy D, Nagel T, Wiesmann M, et al. Identification and characterization of NVP-BKM120, an orally available pan-class I PI3-kinase inhibitor. Mol Cancer Ther. 2012;11:317-28.

105. Bendell JC, Rodon J, Burris HA, de Jonge M, Verweij J, Birle D, Demanse D, De Buck SS, Ru QC, Peters M, et al. Phase I, dose-escalation study of BKM120, an oral pan-Class I PI3K inhibitor, in patients with advanced solid tumors. J Clin Oncol. 2012;30:282-90.

106. Rodon J, Brana I, Siu LL, De Jonge MJ, Homji N, Mills D, Di Tomaso E, Sarr C, Trandafir L, Massacesi C, et al. Phase I dose-escalation and -expansion study of buparlisib (BKM120), an oral pan-Class I PI3K inhibitor, in patients with advanced solid tumors. Invest New Drugs. 2014;32:670-81.

107. Ando Y, Inada-Inoue M, Mitsuma A, Yoshino T, Ohtsu A, Suenaga N, Sato M, Kakizume T, Robson M, Quadt C, Doi T. Phase I dose-escalation study of buparlisib (BKM120), an oral pan-class I PI3K inhibitor, in Japanese patients with advanced solid tumors. Cancer Sci. 2014;105:347-53.

108. Wu YL, Zhang LI, Trandafir L, Dong T, Duval V, Hazell K, Xu B. Phase I Study of the Pan-PI3K Inhibitor Buparlisib in Adult Chinese Patients with Advanced Solid Tumors. Anticancer Res. 2016;36:6185-94.

109. Ragon BK, Kantarjian H, Jabbour E, Ravandi F, Cortes J, Borthakur G, DeBose L, Zeng Z, Schneider H, Pemmaraju N, et al. Buparlisib, a PI3K inhibitor, demonstrates acceptable tolerability and preliminary activity in a phase I trial of patients with advanced leukemias. Am J Hematol. 2017;92:7-11.

110. McRee AJ, Sanoff HK, Carlson C, Ivanova A, O'Neil BH. A phase I trial of mFOLFOX6 combined with the oral PI3K inhibitor BKM120 in patients with advanced refractory solid tumors. Invest New Drugs. 2015;33:1225-31.

111. Hyman DM, Snyder AE, Carvajal RD, Gerecitano JF, Voss MH, Ho AL, Konner J, Winkelmann JL, Stasi MA, Monson KR, et al. Parallel phase lb studies of two schedules of buparlisib (BKM120) plus carboplatin and paclitaxel (q21 days or q28 days) for patients with advanced solid tumors. Cancer Chemother Pharmacol. 2015:75:747-55.

112. Smyth LM, Monson KR, Jhaveri K, Drilon A, Li BT, Abida W, lyer G, Gerecitano JF, Gounder M, Harding JJ, et al. A phase $1 \mathrm{~b}$ dose expansion study of the pan-class I PI3K inhibitor buparlisib (BKM120) plus carboplatin and paclitaxel in PTEN deficient tumors and with dose intensified carboplatin and paclitaxel. Invest New Drugs. 2017;35:742-50.

113. Bedard PL, Tabernero J, Janku F, Wainberg ZA, Paz-Ares L, Vansteenkiste J, Van Cutsem E, Perez-Garcia J, Stathis A, Britten CD, et al. A phase Ib dose-escalation study of the oral pan-PI3K inhibitor buparlisib (BKM120) in combination with the oral MEK1/2 inhibitor trametinib (GSK1120212) in patients with selected advanced solid tumors. Clin Cancer Res. 2015;21:730-8.

114. Matulonis UA, Wulf GM, Barry WT, Birrer M, Westin SN, Farooq S, BellMcGuinn KM, Obermayer E, Whalen C, Spagnoletti T, et al. Phase I dose escalation study of the PI3kinase pathway inhibitor BKM120 and the oral poly (ADP ribose) polymerase (PARP) inhibitor olaparib for the treatment of high-grade serous ovarian and breast cancer. Ann Oncol. 2017;28:512-8.

115. Heudel PE, Fabbro M, Roemer-Becuwe C, Kaminsky MC, Arnaud A, Joly F, RocheForestier S, Meunier J, Foa C, You B, et al. Phase II study of the PI3K inhibitor BKM120 in patients with advanced or recurrent endometrial carcinoma: a stratified type I-type II study from the GINECO group. Br J Cancer. 2017;116:303-9.

116. McKay RR, De Velasco G, Werner L, Bellmunt J, Harshman L, Sweeney C, Rosenberg JE, Hirsch M, Signoretti S, Van Allen EM, et al. A phase 1 study of buparlisib and bevacizumab in patients with metastatic renal cell carcinoma progressing on vascular endothelial growth factor-targeted therapies. Cancer. 2016;122:2389-98.

117. Armstrong AJ, Halabi S, Healy P, Alumkal JJ, Winters C, Kephart J, Bitting RL, Hobbs C, Soleau CF, Beer TM, et al. Phase II trial of the PI3 kinase inhibitor 
buparlisib (BKM-120) with or without enzalutamide in men with metastatic castration resistant prostate cancer. Eur J Cancer. 2017:81:228-36.

118. McRee AJ, Marcom PK, Moore DT, Zamboni WC, Kornblum ZA, Hu Z, Phipps $R$, Anders CK, Reeder-Hayes K, Carey LA, et al. A Phase I Trial of the PI3K Inhibitor Buparlisib Combined With Capecitabine in Patients With Metastatic Breast Cancer. Clin Breast Cancer. 2017.

119. Saura C, Bendell J, Jerusalem G, Su S, Ru Q, De Buck S, Mills D, Ruquet S, Bosch A, Urruticoechea A, et al. Phase lb study of Buparlisib plus Trastuzumab in patients with HER2-positive advanced or metastatic breast cancer that has progressed on Trastuzumab-based therapy. Clin Cancer Res. 2014;20:1935-45.

120. Martin M, Chan A, Dirix L, O'Shaughnessy J, Hegg R, Manikhas A, Shtivelband M, Krivorotko P, Batista Lopez N, Campone M, et al. A randomized adaptive phase II/III study of buparlisib, a pan-class I PI3K inhibitor, combined with paclitaxel for the treatment of HER2- advanced breast cancer (BELLE-4). Ann Oncol. 2017;28:313-20.

121. Loibl S, de la Pena L, Nekljudova V, Zardavas D, Michiels S, Denkert C, Rezai M, Bermejo B, Untch M, Lee SC, et al. Neoadjuvant buparlisib plus trastuzumab and paclitaxel for women with HER2+ primary breast cancer: A randomised, double-blind, placebo-controlled phase II trial (NeoPHOEBE). Eur J Cancer. 2017;85:133-45.

122. Nagaraj G, Ma CX, Luo J, Ellis MJ. A phase I study of BKM120, a novel oral selective phosphatidylinositol-3-kinase (PI3K) inhibitor, in combination with fulvestrant in postmenopausal women with estrogen receptor positive metastatic breast cancer. J Clin Oncol. 2012;30:TPS664-TPS664.

123. Baselga J, Im SA, Iwata H, Cortes J, De Laurentiis M, Jiang Z, Arteaga CL, Jonat W, Clemons M, Ito Y, et al. Buparlisib plus fulvestrant versus placebo plus fulvestrant in postmenopausal, hormone receptor-positive, HER2negative, advanced breast cancer (BELLE-2): a randomised, double-blind, placebo-controlled, phase 3 trial. Lancet Oncol. 2017;18:904-16.

124. Di Leo A, Johnston S, Lee KS, Ciruelos E, Lonning PE, Janni W, O'Regan R, MouretReynier MA, Kalev D, Egle D, et al. Buparlisib plus fulvestrant in postmenopausal women with hormone-receptor-positive, HER2-negative, advanced breast cancer progressing on or after mTOR inhibition (BELLE-3): a randomised, double-blind, placebo-controlled, phase 3 trial. Lancet Oncol. 2018;19:87-100.

125. Liu N, Rowley BR, Bull CO, Schneider C, Haegebarth A, Schatz CA, Fracasso PR, Wilkie DP, Hentemann M, Wilhelm SM, et al. BAY 80-6946 is a highly selective intravenous PI3K inhibitor with potent p110alpha and p110delta activities in tumor cell lines and xenograft models. Mol Cancer Ther. 2013;12:2319-30.

126. Patnaik A, Appleman LJ, Tolcher AW, Papadopoulos KP, Beeram M, Rasco DW, Weiss GJ, Sachdev JC, Chadha M, Fulk M, et al. First-in-human phase I study of copanlisib (BAY 80-6946), an intravenous pan-class I phosphatidylinositol 3-kinase inhibitor, in patients with advanced solid tumors and non-Hodgkin's lymphomas. Ann Oncol. 2016;27:1928-40.

127. Doi T, Fuse N, Yoshino T, Kojima T, Bando H, Miyamoto H, Kaneko M, Osada M, Ohtsu A. A Phase I study of intravenous PI3K inhibitor copanlisib in Japanese patients with advanced or refractory solid tumors. Cancer Chemother Pharmacol. 2017;79:89-98.

128. Kim RD, Alberts SR, Pena C, Genvresse I, Ajavon-Hartmann A, Xia C, Kelly A, Grilley-Olson JE. Phase I dose-escalation study of copanlisib in combination with gemcitabine or cisplatin plus gemcitabine in patients with advanced cancer. Br J Cancer. 2018;118:462-70.

129. Dreyling M, Morschhauser F, Bouabdallah K, Bron D, Cunningham D, Assouline SE, Verhoef G, Linton K, Thieblemont C, Vitolo U, et al. Phase II study of copanlisib, a PI3K inhibitor, in relapsed or refractory, indolent or aggressive lymphoma. Ann Oncol. 2017;28:2169-78.

130. Dreyling M, Santoro A, Mollica L, Leppa S, Follows GA, Lenz G, Kim WS, Nagler A, Panayiotidis P, Demeter J, et al. Phosphatidylinositol 3-Kinase Inhibition by Copanlisib in Relapsed or Refractory Indolent Lymphoma. J Clin Oncol. 2017;35:3898-905.

131. Markham A. Copanlisib: First Global Approval. Drugs. 2017;77:2057-62.

132. Dong S, Guinn D, Dubovsky JA, Zhong Y, Lehman A, Kutok J, Woyach JA, Byrd JC, Johnson AJ. IPI-145 antagonizes intrinsic and extrinsic survival signals in chronic lymphocytic leukemia cells. Blood. 2014;124:3583-6.

133. Horwitz SM, Koch R: Activity of the PI3K-delta,gamma inhibitor duvelisib in a phase 1 trial and preclinical models of T-cell lymphoma. Blood. 2018;131:888-98.

134. Patel VM, Balakrishnan K, Douglas M, Tibbitts T, Xu EY, Kutok JL, Ayers M, Sarkar A, Guerrieri R, Wierda WG, et al. Duvelisib treatment is associated with altered expression of apoptotic regulators that helps in sensitization of chronic lymphocytic leukemia cells to venetoclax (ABT-199). Leukemia. 2017; 31:1872-81.
135. Flinn I, Oki Y, Patel M, Horwitz SM, Foss FM, Sweeney J, Allen K, Douglas M, Steelman L, Dunbar J, et al. A Phase 1 Evaluation of Duvelisib (IPI-145), a PI3K- $\delta, y$ Inhibitor, in Patients with Relapsed/Refractory iNHL. Blood. 2014; 124:802.

136. Flinn IW, Miller CB, Ardeshna KM, Tetreault S, Assouline SE, Zinzani PL, Mayer J, Merli M, Lunin SD, Pettitt AR, et al. Dynamo: a phase 2 study demonstrating the clinical activity of duvelisib in patients with relapsed refractory indolent non-hodgkin lymphoma. Blood Conference: 58th annual meeting of the american society of hematology, ASH; 2016. p. 128.

137. Flinn IW, Hillmen P, Montillo M, Nagy Z, Illes A, Etienne G, Delgado J, Kuss BJ, Tam CS, Gasztonyi Z, et al. The phase 3 DUO trial: duvelisib vs ofatumumab in relapsed and refractory CLL/SLL. Blood. 2018;132:2446-55.

138. Liu P, Cheng H, Roberts TM, Zhao JJ. Targeting the phosphoinositide 3kinase pathway in cancer. Nat Rev Drug Discov. 2009:8:627-44.

139. Workman P, Clarke PA, Raynaud FI, van Montfort RL. Drugging the PI3 kinome: from chemical tools to drugs in the clinic. Cancer Res. 2010;70: 2146-57.

140. O'Brien C, Wallin JJ, Sampath D, GuhaThakurta D, Savage H, Punnoose EA, Guan J, Berry L, Prior WW, Amler LC, et al. Predictive biomarkers of sensitivity to the phosphatidylinositol 3' kinase inhibitor GDC-0941 in breast cancer preclinical models. Clin Cancer Res. 2010;16:3670-83.

141. Raynaud Fl, Eccles SA, Patel S, Alix S, Box G, Chuckowree I, Folkes A, Gowan S, De Haven BA, Di Stefano F, et al. Biological properties of potent inhibitors of class I phosphatidylinositide 3-kinases: from PI-103 through PI-540, PI-620 to the oral agent GDC-0941. Mol Cancer Ther. 2009;8:1725-38.

142. Sarker D, Ang JE, Baird R, Kristeleit R, Shah K, Moreno V, Clarke PA, Raynaud FI, Levy G, Ware JA, et al. First-in-human phase I study of pictilisib (GDC0941), a potent pan-class I phosphatidylinositol-3-kinase (PI3K) inhibitor, in patients with advanced solid tumors. Clin Cancer Res. 2015;21:77-86.

143. Yamamoto N, Fujiwara Y, Tamura K, Kondo S, Iwasa S, Tanabe Y, Horiike A, Yanagitani N, Kitazono S, Inatani M, et al. Phase la/lb study of the pan-class I PI3K inhibitor pictilisib (GDC-0941) administered as a single agent in Japanese patients with solid tumors and in combination in Japanese patients with non-squamous non-small cell lung cancer. Invest New Drugs. 2017;35:37-46.

144. Leong S, Moss RA, Bowles DW, Ware JA, Zhou J, Spoerke JM, Lackner MR, Shankar G, Schutzman JL, van der Noll R, et al. A Phase I Dose-Escalation Study of the Safety and Pharmacokinetics of Pictilisib in Combination with Erlotinib in Patients with Advanced Solid Tumors. Oncologist. 2017;22:1491-9.

145. Soria JC, Adjei AA, Bahleda R, Besse B, Ferte C, Planchard D, Zhou J, Ware J, Morrissey K, Shankar G, et al. A phase IB dose-escalation study of the safety and pharmacokinetics of pictilisib in combination with either paclitaxel and carboplatin (with or without bevacizumab) or pemetrexed and cisplatin (with or without bevacizumab) in patients with advanced non-small cell lung cancer. Eur J Cancer. 2017:86:186-96.

146. Krop IE, Mayer IA, Ganju V, Dickler M, Johnston S, Morales S, Yardley DA, Melichar B, Forero-Torres A, Lee SC, et al. Pictilisib for oestrogen receptorpositive, aromatase inhibitor-resistant, advanced or metastatic breast cancer (FERGI): a randomised, double-blind, placebo-controlled, phase 2 trial. Lancet Oncol. 2016;17:811-21.

147. Vuylsteke $P$, Huizing M, Petrakova K, Roylance R, Laing R, Chan S, Abell F, Gendreau S, Rooney I, Apt D, et al. Pictilisib PI3Kinase inhibitor (a phosphatidylinositol 3-kinase [PI3K] inhibitor) plus paclitaxel for the treatment of hormone receptor-positive, HER2-negative, locally recurrent, or metastatic breast cancer: interim analysis of the multicentre, placebocontrolled, phase II randomised PEGGY study. Sci Signal. 2016;27:2059-66.

148. Schmid P, Pinder SE, Wheatley D, Macaskill J, Zammit C, Hu J, Price R, Bundred N, Hadad S, Shia A, et al. Phase II Randomized Preoperative Window-of-Opportunity Study of the PI3K Inhibitor Pictilisib Plus Anastrozole Compared With Anastrozole Alone in Patients With Estrogen Receptor-Positive Breast Cancer. J Clin Oncol. 2016;34:1987-94.

149. Tamura K, Kodaira M, Shimizu C, Yonemori K, Yunokawa M, Shimomura A, Kobayashi T, Nakano K, Tomomatsu J, Ito Y, et al. Phase I study of taselisib in Japanese patients with advanced solid tumors or hormone receptorpositive advanced breast cancer. Cancer Sci. 2018;109:1592-601.

150. Juric D, Krop I, Ramanathan RK, Wilson TR, Ware JA, Sanabria Bohorquez SM, Savage HM, Sampath D, Salphati L, Lin RS, et al. Phase I Dose-Escalation Study of Taselisib, an Oral PI3K Inhibitor, in Patients with Advanced Solid Tumors. Cancer Discov. 2017;7:704-15.

151. Fritsch C, Huang A, Chatenay-Rivauday C, Schnell C, Reddy A, Liu M, Kauffmann A, Guthy D, Erdmann D, De Pover A, et al. Characterization of 
the novel and specific PI3Kalpha inhibitor NVP-BYL719 and development of the patient stratification strategy for clinical trials. Mol Cancer Ther. 2014;13: $1117-29$.

152. Elkabets M, Vora S, Juric D, Morse N, Mino-Kenudson M, Muranen T, Tao J, Campos AB, Rodon J, Ibrahim YH, et al. mTORC1 inhibition is required for sensitivity to PI3K p110alpha inhibitors in PIK3CA-mutant breast cancer. Sci Transl Med. 2013;5:196ra199.

153. Juric D, Rodon J, Tabernero J, Janku F, Burris HA, Schellens JHM, Middleton MR, Berlin J, Schuler M, Gil-Martin M, et al. Phosphatidylinositol 3-Kinase alphaSelective Inhibition With Alpelisib (BYL719) in PIK3CA-Altered Solid Tumors: Results From the First-in-Human Study. J Clin Oncol. 2018;36:1291-9.

154. Mayer IA, Abramson VG, Formisano L, Balko JM, Estrada MV, Sanders ME, Juric D, Solit D, Berger MF, Won HH, et al. A Phase lb Study of Alpelisib (BYL719), a PI3Kalpha-Specific Inhibitor, with Letrozole in ER+/HER2Metastatic Breast Cancer. Clin Cancer Res. 2017;23:26-34.

155. Jain S, Shah AN, Santa-Maria CA, Siziopikou K, Rademaker A, Helenowski I, Cristofanilli M, Gradishar WJ. Phase I study of alpelisib (BYL-719) and trastuzumab emtansine (T-DM1) in HER2-positive metastatic breast cancer (MBC) after trastuzumab and taxane therapy. Breast Cancer Res Treat. 2018; 171:371-381.

156. van Geel R, Tabernero J, Elez E, Bendell JC, Spreafico A, Schuler M, Yoshino T, Delord JP, Yamada Y, Lolkema MP, et al. A Phase lb Dose-Escalation Study of Encorafenib and Cetuximab with or without Alpelisib in Metastatic BRAFMutant Colorectal Cancer. Cancer Discov. 2017;7:610-9.

157. Gopal AK, Kahl BS, de Vos S, Wagner-Johnston ND, Schuster SJ, Jurczak WJ, Flinn IW, Flowers CR, Martin P, Viardot A, et al. PI3Kdelta inhibition by idelalisib in patients with relapsed indolent lymphoma. N Engl J Med. 2014; 370:1008-18.

158. Herman SE, Gordon AL, Wagner AJ, Heerema NA, Zhao W, Flynn JM, Jones J, Andritsos L, Puri KD, Lannutti BJ, et al. Phosphatidylinositol 3-kinase-delta inhibitor CAL-101 shows promising preclinical activity in chronic lymphocytic leukemia by antagonizing intrinsic and extrinsic cellular survival signals. Blood. 2010;116:2078-88.

159. Flinn IW, Kahl BS, Leonard JP, Furman RR, Brown JR, Byrd JC, WagnerJohnston ND, Coutre SE, Benson DM, Peterman S, et al. Idelalisib, a selective inhibitor of phosphatidylinositol 3-kinase-delta, as therapy for previously treated indolent non-Hodgkin lymphoma. Blood. 2014;123:3406-13.

160. Kahl BS, Spurgeon SE, Furman RR, Flinn IW, Coutre SE, Brown JR, Benson DM, Byrd JC, Peterman S, Cho Y, et al. A phase 1 study of the PI3Kdelta inhibitor idelalisib in patients with relapsed/refractory mantle cell lymphoma (MCL). Blood. 2014;123:3398-405.

161. Brown JR, Byrd JC, Coutre SE, Benson DM, Flinn IW, Wagner-Johnston ND, Spurgeon SE, Kahl BS, Bello C, Webb HK, et al. Idelalisib, an inhibitor of phosphatidylinositol 3-kinase p110delta, for relapsed/refractory chronic lymphocytic leukemia. Blood. 2014;123:3390-7.

162. Lampson BL, Kasar SN, Matos TR, Morgan EA. Idelalisib given front-line for treatment of chronic lymphocytic leukemia causes frequent immunemediated hepatotoxicity. Blood. 2016;128:195-203.

163. Smith SM, Pitcher BN, Jung SH, Bartlett NL, Wagner-Johnston N, Park SI, Richards KL, Cashen AF, Jaslowski A, Smith SE, et al. Safety and tolerability of idelalisib, lenalidomide, and rituximab in relapsed and refractory lymphoma: the Alliance for Clinical Trials in Oncology A051201 and A051202 phase 1 trials. Lancet Haematol. 2017:4:e176-82.

164. Gopal AK, Fanale MA, Moskowitz CH, Shustov AR, Mitra S, Ye W, Younes A, Moskowitz AJ. Phase II study of idelalisib, a selective inhibitor of PI3Kdelta, for relapsed/refractory classical Hodgkin lymphoma. Ann Oncol. 2017;28:1057-63.

165. O'Brien SM, Lamanna N, Kipps TJ, Flinn I, Zelenetz AD, Burger JA, Keating M, Mitra S, Holes L, Yu AS, et al. A phase 2 study of idelalisib plus rituximab in treatment-naive older patients with chronic lymphocytic leukemia. Blood. 2015;126:2686-94.

166. Furman RR, Sharman JP, Coutre SE, Cheson BD, Pagel JM, Hillmen P, Barrientos JC, Zelenetz AD, Kipps TJ, Flinn I, et al. Idelalisib and rituximab in relapsed chronic lymphocytic leukemia. New England J Med. 2014;370:997-1007.

167. Jones JA, Robak T, Brown JR, Awan FT, Badoux X, Coutre S, Loscertales J, Taylor K, Vandenberghe E, Wach M, et al. Efficacy and safety of idelalisib in combination with ofatumumab for previously treated chronic lymphocytic leukaemia: an open-label, randomised phase 3 trial. Proc Natl Acad Sci U S A. 2017:4:e114-26.

168. Huw LY, O'Brien C, Pandita A. Acquired PIK3CA amplification causes resistance to selective phosphoinositide 3-kinase inhibitors in breast cancer. Oncogenesis. 2013;2:e83.
169. Nakanishi Y, Walter K, Spoerke JM, O'Brien C, Huw LY, Hampton GM, Lackner MR. Activating Mutations in PIK3CB Confer Resistance to PI3K Inhibition and Define a Novel Oncogenic Role for p110beta. Cancer Res. 2016;76:1193-203.

170. Jia S, Liu Z, Zhang S, Liu P, Zhang L, Lee SH, Zhang J, Signoretti S, Loda M, Roberts TM, Zhao JJ. Essential roles of PI(3)K-p110beta in cell growth, metabolism and tumorigenesis. Nature. 2008;454:776-9.

171. Edgar KA, Wallin JJ, Berry M, Lee LB, Prior WW, Sampath D, Friedman LS, Belvin M. Isoform-specific phosphoinositide 3-kinase inhibitors exert distinct effects in solid tumors. Cancer Res. 2010;70:1164-72.

172. Juric $D$, Castel $P$, Griffith M, Griffith $\mathrm{OL}$, Won HH, Ellis $\mathrm{H}$, Ebbesen $\mathrm{SH}$, Ainscough BJ, Ramu A, lyer G, et al. Convergent loss of PTEN leads to clinical resistance to a PI(3)Kalpha inhibitor. Nature. 2015;518:240-4.

173. Han MW, Ryu IS, Lee JC, Kim SH, Chang HW, Lee YS, Lee S, Kim SW, Kim SY. Phosphorylation of PI3K regulatory subunit p85 contributes to resistance against PI3K inhibitors in radioresistant head and neck cancer. Oral Oncol. 2018;78:56-63.

174. Castellano E, Downward J. RAS Interaction with PI3K: More Than Just Another Effector Pathway. Genes Cancer. 2011;2:261-74.

175. Ruicci KM, Pinto N, Khan MI, Yoo J, Fung K, MacNeil D, Mymryk JS, Barrett JW, Nichols AC. ERK-TSC2 signalling in constitutively-active HRAS mutant HNSCC cells promotes resistance to PI3K inhibition. Oral Oncol. 2018;84:95103.

176. Mundt F, Rajput S, Li S, Ruggles KV, Mooradian AD, Mertins P, Gillette MA, Krug K, Guo Z, Hoog J, et al. Mass Spectrometry-Based Proteomics Reveals Potential Roles of NEK9 and MAP2K4 in Resistance to PI3K Inhibition in Triple-Negative Breast Cancers. Cancer Res. 2018;78:2732-46.

177. Chakrabarty A, Sanchez V, Kuba MG, Rinehart C, Arteaga CL. Feedback upregulation of HER3 (ErbB3) expression and activity attenuates antitumor effect of PI3K inhibitors. Proc Natl Acad Sci U S A. 2012;109:2718-23.

178. Serra V, Scaltriti M, Prudkin L, Eichhorn PJ, Ibrahim YH, Chandarlapaty S, Markman B, Rodriguez O, Guzman M, Rodriguez S, et al. PI3K inhibition results in enhanced HER signaling and acquired ERK dependency in HER2overexpressing breast cancer. Oncogene. 2011;30:2547-57.

179. Okada K, Nogami A, Ishida S, Akiyama H, Chen C, Umezawa Y, Miura O. FLT3-ITD induces expression of Pim kinases through STAT5 to confer resistance to the PI3K/Akt pathway inhibitors on leukemic cells by enhancing the mTORC1/mcl-1 pathway. Oncotarget. 2018;9:8870-86.

180. Britschgi A, Andraos R, Brinkhaus H, Klebba I, Romanet V, Muller U, Murakami M, Radimerski T, Bentires-Alj M. JAK2/STAT5 inhibition circumvents resistance to PI3K/mTOR blockade: a rationale for cotargeting these pathways in metastatic breast cancer. Cancer Cell. 2012;22:796-811.

181. Yang L, Han S, Sun Y. An IL6-STAT3 loop mediates resistance to PI3K inhibitors by inducing epithelial-mesenchymal transition and cancer stem cell expansion in human breast cancer cells. Biochem Biophys Res Commun. 2014:453:582-7.

182. Park YL, Kim HP, Cho YW, Min DW, Cheon SK, Lim YJ, Song SH, Jin Kim S, Han SW, Park KJ, Kim TY. Activation of WNT/beta-catenin signaling results in resistance to a dual $\mathrm{PI} 3 \mathrm{~K} / \mathrm{mTOR}$ inhibitor in colorectal cancer cells harboring PIK3CA mutations. Int J Cancer. 2018.

183. Tenbaum SP, Ordonez-Moran P, Puig I, Chicote I, Arques O, Landolfi S, Fernandez $Y$, Herance JR, Gispert JD, Mendizabal L, et al. beta-catenin confers resistance to $\mathrm{PI} 3 \mathrm{~K}$ and AKT inhibitors and subverts FOXO3a to promote metastasis in colon cancer. Nat Med. 2012;18:892-901.

184. Dey N, Leyland-Jones B, De P. MYC-xing it up with PIK3CA mutation and resistance to PI3K inhibitors: summit of two giants in breast cancers. Am J Cancer Res. 2015;5:1-19.

185. Muellner MK, Uras IZ, Gapp BV, Kerzendorfer C, Smida M, Lechtermann H, Craig-Mueller N, Colinge J, Duernberger G, Nijman SM. A chemical-genetic screen reveals a mechanism of resistance to PI3K inhibitors in cancer. Nat Chem Biol. 2011;7:787-93.

186. Ilic N, Utermark T, Widlund HR, Roberts TM. PI3K-targeted therapy can be evaded by gene amplification along the MYC-eukaryotic translation initiation factor 4E (elF4E) axis. Proc Natl Acad Sci U S A. 2011;108:E699-708.

187. Le X, Antony R, Razavi P, Treacy DJ, Luo F, Ghandi M, Castel P, Scaltriti M, Baselga J, Garraway LA. Systematic functional characterization of resistance to PI3K inhibition in breast Cancer. Cancer Discov. 2016;6:1134-47.

188. Song JH, Singh N, Luevano LA, Padi SKR, Okumura K, Olive V, Black SM, Warfel NA, Goodrich DW. Mechanisms behind resistance to PI3K inhibitor treatment induced by the PIM kinase; 2018.

189. Clement E. Skp2-dependent reactivation of AKT drives resistance to PI3K inhibitors. Ther Adv Med Oncol. 2018;11. 
190. Serra V, Eichhorn PJ, Garcia-Garcia C, Ibrahim YH, Prudkin L, Sanchez G, Rodriguez O, Anton P, Parra JL, Marlow S, et al. RSK3/4 mediate resistance to PI3K pathway inhibitors in breast cancer. J Clin Invest. 2013;123:2551-63.

191. Wang W, Lim KG, Feng M, Bao Y, Lee PL, Yu C, Chen Y, Zhang H, Marzese D, Hoon DS, Yu Q. KDM6B counteracts EZH2-mediated suppression of IGFBP5 to confer resistance to PI3K/AKT inhibitor treatment in breast cancer. Mol Cancer Ther. 2018.

192. Vora SR, Juric D, Kim N, Mino-Kenudson M, Huynh T, Costa C, Lockerman EL, Pollack SF, Liu M, Li X, et al. CDK 4/6 inhibitors sensitize PIK3CA mutant breast cancer to PI3K inhibitors. Cancer Cell. 2014;26:136-49.

193. Makinoshima H, Umemura S, Suzuki A, Nakanishi H, Maruyama A, Udagawa H, Mimaki S, Matsumoto S, Niho S, Ishii G, et al. Metabolic determinants of sensitivity to phosphatidylinositol 3-kinase pathway inhibitor in small-cell lung carcinoma. Cancer Res. 2018;78:2179-90.

194. Yu B, Ye X, Du Q, Zhu B, Zhai Q, Li XX. The long non-coding RNA CRNDE promotes colorectal carcinoma progression by competitively binding miR217 with TCF7L2 and enhancing the Wnt/beta-catenin signaling pathway. Cell Physiol Biochem. 2017;41:2489-502.

195. Gong X, Huang M. Long non-coding RNA MEG3 promotes the proliferation of glioma cells through targeting Wnt/beta-catenin signal pathway. Cancer Gene Ther. 2017;24:381-5.

196. Chen L, Feng P, Zhu X, He S, Duan J, Zhou D. Long non-coding RNA Malat1 promotes neurite outgrowth through activation of ERK/MAPK signalling pathway in N2a cells. J Cell Mol Med. 2016;20:2102-10.

197. Hu YC, Wang AM, Lu JK, Cen R, Liu LL. Long noncoding RNA HOXD-AS1 regulates proliferation of cervical cancer cells by activating Ras/ERK signaling pathway. Eur Rev Med Pharmacol Sci. 2017;21:5049-55.

198. Reicher A, Fosselteder J, Kwong LN, Pichler M. Crosstalk between the Notch signaling pathway and long non-coding RNAs. Cancer Lett. 2018;420:91-6.

199. Wang S, Liang K, Hu Q, Li P, Song J, Yang Y, Yao J, Mangala LS, Li C, Yang $W$, et al. JAK2-binding long noncoding RNA promotes breast cancer brain metastasis. J Clin Invest. 2017:127:4498-515.

200. Wan L, Tian Y, Zhang R, Peng Z, Sun J, Zhang W. MicroRNA-103 confers the resistance to long-treatment of adriamycin to human leukemia cells by regulation of COP1. J Cell Biochem. 2018;119:3843-52.

201. Song L, Zhou Z, Gan Y, Li P, Xu Y, Zhang Z, Luo F, Xu J, Zhou Q, Dai F. Long noncoding RNA OIP5-AS1 causes cisplatin resistance in osteosarcoma through inducing the LPAATbeta/PI3K/AKT/mTOR signaling pathway by sponging the miR-340-5p. J Cell Biochem. 2018.

202. Cheng C, Qin Y, Zhi Q, Wang J, Qin C. Knockdown of long non-coding RNA HOTAIR inhibits cisplatin resistance of gastric cancer cells through inhibiting the PI3K/Akt and Wnt/beta-catenin signaling pathways by up-regulating miR-34a. Int J Biol Macromol. 2018;107:2620-9.

203. Wang H, Li Q, Tang S, Li M, Feng A, Qin L, Liu Z, Wang X. The role of long noncoding RNA HOTAIR in the acquired multidrug resistance to imatinib in chronic myeloid leukemia cells. Hematology. 2017;22:208-16.

204. Wang BD, Ceniccola K, Hwang S, Andrawis R, Horvath A, Freedman JA, Olender J, Knapp S, Ching T, Garmire L, et al. Alternative splicing promotes tumour aggressiveness and drug resistance in African American prostate cancer. Nat Commun. 2017:8:15921.

205. Liu B, Liu Y, Zhao L, Pan Y, Shan Y, Li Y, Jia L. Upregulation of microRNA-135b and microRNA-182 promotes chemoresistance of colorectal cancer by targeting ST6GALNAC2 via PI3KJAKT pathway. Mol Carcinog. 2017;56:2669-80.

206. Li Y, Ye Y, Feng B, Qi Y. Long noncoding RNA IncARSR promotes doxorubicin resistance in hepatocellular carcinoma via modulating PTENPI3K/Akt pathway. J Cell Biochem. 2017;118:4498-507.

207. Zhou JY, Chen X, Zhao J, Bao Z, Chen X, Zhang P, Liu ZF, Zhou JY. MicroRNA-34a overcomes HGF-mediated gefitinib resistance in EGFR mutant lung cancer cells partly by targeting MET. Cancer Lett. 2014;351: 265-71

208. Xie Q, Yan Y, Huang Z, Zhong X, Huang L. MicroRNA-221 targeting PI3-K Akt signaling axis induces cell proliferation and BCNU resistance in human glioblastoma. Neuropathology. 2014;34:455-64.

209. Qian XJ, Li YT, Yu Y, Yang F, Deng R, Ji J, Jiao L, Li X, Wu RY, Chen WD, et al. Inhibition of DNA methyltransferase as a novel therapeutic strategy to overcome acquired resistance to dual PI3K/mTOR inhibitors. Oncotarget. 2015;6:5134-46.

210. Koh KX, Tan GH, Hui Low SH, Mohd Omar MF, Han MJ, lacopetta B, Soo R, Beloueche-Babari M, Bhattacharya B, Soong R. Acquired resistance to PI3K mTOR inhibition is associated with mitochondrial DNA mutation and glycolysis. Oncotarget. 2017:8:110133-44.
211. Usman MW, Gao J, Zheng T, Rui C, Li T, Bian X, Cheng H, Liu P, Luo F. Macrophages confer resistance to PI3K inhibitor GDC-0941 in breast cancer through the activation of NF-kappaB signaling. Cell Death Dis. 2018;9:809.

212. Josephs DH, Sarker D. Pharmacodynamic biomarker development for PI3K pathway therapeutics. Transl Oncogenomics. 2015;7:33-49.

213. Yang $N$, Chen J, Zhang $H$, Wang $X$, Yao H, Peng Y, Zhang W. LncRNA OIP5AS1 loss-induced microRNA-410 accumulation regulates cell proliferation and apoptosis by targeting KLF10 via activating PTEN/PI3KNAKT pathway in multiple myeloma. Cell Death Dis. 2017;8:e2975.

214. Yang T, Zhai H, Yan R, Zhou Z, Gao L, Wang L. IncRNA CCAT1 promotes cell proliferation, migration, and invasion by down-regulation of miR-143 in FTC-133 thyroid carcinoma cell line. Braz J Med Biol Res. 2018:51:e7046.

215. Jiang Y, Jiang YY, Xie JJ, Mayakonda A, Hazawa M, Chen L, Xiao JF, Li CQ, Huang ML, Ding LW, Sun QY. Co-activation of super-enhancer-driven CCAT1 by TP63 and SOX2 promotes squamous cancer progression. Nat Commun. 2018:9:3619.

216. Qi D, Wang M, Yu F. Knockdown of IncRNA-H19 inhibits cell viability, migration and invasion while promotes apoptosis via microRNA-143/RUNX2 axis in retinoblastoma. Biomed Pharmacother. 2019;109:798-805.

217. Liao Z, Zhao J, Yang Y. Downregulation of IncRNA H19 inhibits the migration and invasion of melanoma cells by inactivating the NFkappaB and PI3K/Akt signaling pathways. Mol Med Rep. 2018;17:7313-8.

218. Li Z, Luo J. Epigenetic regulation of HOTAIR in advanced chronic myeloid leukemia. Cancer Manag Res. 2018;10:5349-62.

219. Luan W, Li R, Liu L, Ni X, Shi Y, Xia Y, Wang J, Lu F, Xu B. Long non-coding RNA HOTAIR acts as a competing endogenous RNA to promote malignant melanoma progression by sponging miR-152-3p. Oncotarget. 2017;8:8540114.

220. Yan J, Dang Y, Liu S, Zhang Y, Zhang G. LncRNA HOTAIR promotes cisplatin resistance in gastric cancer by targeting miR-126 to activate the PI3KAKT/ MRP1 genes. Tumour Biol. 2016.

221. Hui Z, Xianglin M. Association of HOTAIR expression with PI3K/Akt pathway activation in adenocarcinoma of esophagogastric junction. Open Med (Wars). 2016;11:36-40.

222. Ke J, Yao YL, Zheng J, Wang P, Liu YH, Ma J, Li Z, Liu XB, Li ZQ, Wang ZH, Xue YX. Knockdown of long non-coding RNA HOTAIR inhibits malignant biological behaviors of human glioma cells via modulation of miR-326. Oncotarget. 2015:6:21934-49.

223. Xu H, Li J, Zhou ZG. NEAT1 promotes cell proliferation in multiple myeloma by activating PI3KJAKT pathway. Eur Rev Med Pharmacol Sci. 2018;22:640311.

224. Wang HL, Hou SY, Li HB, Qiu JP, Bo L, Mao CP. Biological function and mechanism of long noncoding RNAs nuclear-enriched abundant transcript 1 in development of cervical Cancer. Chin Med J. 2018;131:2063-70.

225. Wang CL, Wang D, Yan BZ, Fu JW, Qin L. Long non-coding RNA NEAT1 promotes viability and migration of gastric cancer cell lines through upregulation of microRNA-17. Eur Rev Med Pharmacol Sci. 2018;22:4128-37.

226. Guo HM, Yang SH, Zhao SZ, Li L, Yan MT, Fan MC. LncRNA NEAT1 regulates cervical carcinoma proliferation and invasion by targeting AKT/PI3K. Eur Rev Med Pharmacol Sci. 2018:22:4090-7.

227. Xin X, Wu M, Meng Q, Wang C, Lu Y, Yang Y, Li X, Zheng Q, Pu H, Gui X, et al. Long noncoding RNA HULC accelerates liver cancer by inhibiting PTEN via autophagy cooperation to miR15a. Mol Cancer. 2018;17:94.

228. Ma J, Ding Y. Geniposide suppresses growth, migration and invasion of MKN45 cells by down-regulation of IncRNA HULC. Exp Mol Pathol. 2018;105: 252-9.

229. Kong D, Wang Y. Knockdown of IncRNA HULC inhibits proliferation, migration, invasion, and promotes apoptosis by sponging miR-122 in osteosarcoma. J Cell Biochem. 2018;119:1050-1061.

230. Wang J, Ma W, Liu Y. Long non-coding RNA HULC promotes bladder cancer cells proliferation but inhibits apoptosis via regulation of ZIC2 and PI3K/AKT signaling pathway. Mol Cancer. 2017;20:425-34.

231. Lu Y, Li Y, Chai X, Kang Q, Zhao P, Xiong J, Wang J. Long noncoding RNA HULC promotes cell proliferation by regulating PI3K AKT signaling pathway in chronic myeloid leukemia. J Cell Biochem. 2017;607:41-6.

232. Zhu Y, Zhang X, Qi L, Cai Y, Yang P, Xuan G, Jiang Y. HULC long noncoding RNA silencing suppresses angiogenesis by regulating ESM-1 via the PI3K/Akt/ mTOR signaling pathway in human gliomas. Oncotarget. 2016;7:14429-40.

233. Wang Y, Kuang $H$, Xue J, Liao L, Yin F, Zhou X. LncRNA AB073614 regulates proliferation and metastasis of colorectal cancer cells via the PI3K/AKT signaling pathway. Biomed Pharmacother. 2017;93:1230-7. 
234. Huang JL, Cao SW, Ou QS, Yang B, Zheng SH, Tang J, Chen J, Hu YW, Zheng $\mathrm{L}$, Wang $\mathrm{Q}$. The long non-coding RNA PTTG3P promotes cell growth and metastasis via up-regulating PTTG1 and activating PI3K/AKT signaling in hepatocellular carcinoma. Mol Cancer. 2018;17:93.

235. Wang N, Hou MS, Zhan Y, Shen XB, Xue HY. MALAT1 promotes cisplatin resistance in cervical cancer by activating the PI3K/AKT pathway. Eur Rev Med Pharmacol Sci. 2018;22:7653-9.

236. Liu D, Zhu Y, Pang J, Weng X, Feng X, Guo Y. Knockdown of long noncoding RNA MALAT1 inhibits growth and motility of human hepatoma cells via modulation of miR-195. J Cell Biochem. 2018;119:1368-80.

237. Li Z, Ma Z, Xu X. Long noncoding RNA MALAT1 correlates with cell viability and mobility by targeting miR223p in renal cell carcinoma via the PI3K/Akt pathway. Oncol Rep. 2018;41(2):1113-21.

238. He B, Peng F, Li W, Jiang Y. Interaction of IncRNA-MALAT1 and miR-124 regulates $\mathrm{HBX}$-induced cancer stem cell properties in HepG2 through PI3K Akt signaling; 2018.

239. Chen Y, Huang W, Sun W, Zheng B, Wang C, Luo Z, Wang J, Yan W. LncRNA MALAT1 promotes Cancer metastasis in osteosarcoma via activation of the PI3K-Akt signaling pathway. J Cell Biochem. 2018:51:1313-26.

240. Wang C, Mao ZP, Wang L, Wu GH, Zhang FH, Wang DY, Shi JL. Long noncoding RNA MALAT1 promotes cholangiocarcinoma cell proliferation and invasion by activating PI3K/Akt pathway. Neoplasma. 2017;64:725-31.

241. Jin Y, Feng SJ, Qiu S, Shao N, Zheng JH. LncRNA MALAT1 promotes proliferation and metastasis in epithelial ovarian cancer via the PI3K-AKT pathway. Eur Rev Med Pharmacol Sci. 2017;21:3176-84

242. Meseure D, Vacher S, Lallemand F, Alsibai KD, Hatem R, Chemlali W, Nicolas A, De Koning L, Pasmant E, Callens C, et al. Prognostic value of a newly identified MALAT1 alternatively spliced transcript in breast cancer. Br J Cancer. 2016;114:1395-404.

243. Xu S, Sui S, Zhang J, Bai N, Shi Q, Zhang G, Gao S, You Z, Zhan C, Liu F, Pang D. Downregulation of long noncoding RNA MALAT1 induces epithelial-to-mesenchymal transition via the PI3K-AKT pathway in breast cancer. Int J Clin Exp Pathol. 2015;8:4881-91.

244. Dong Y, Liang G, Yuan B, Yang C, Gao R, Zhou X. MALAT1 promotes the proliferation and metastasis of osteosarcoma cells by activating the PI3K/Akt pathway. Tumour Biol. 2015;36:1477-86.

245. Zhang X, He X, Liu Y, Zhang H, Chen H, Guo S, Liang Y. MiR-101-3p inhibits the growth and metastasis of non-small cell lung cancer through blocking PI3K/AKT signal pathway by targeting MALAT-1. Biomed Pharmacother. 2017:93:1065-73.

246. Zhai X, Xu W. Long noncoding RNA ATB promotes proliferation, migration and invasion in bladder cancer by suppressing microRNA-126. Oncol Res. 2018.

247. Xu S, Yi XM, Tang CP, Ge JP, Zhang ZY, Zhou WQ. Long non-coding RNA ATB promotes growth and epithelial-mesenchymal transition and predicts poor prognosis in human prostate carcinoma. Oncol Rep. 2016;36:10-22.

248. Pan H, Jiang T, Cheng N, Wang Q, Ren S, Li X, Zhao C, Zhang L, Cai W, Zhou C. Long non-coding RNA BC087858 induces non-T790M mutation acquired resistance to EGFR-TKIs by activating PI3K/AKT and MEK/ERK pathways and EMT in non-small-cell lung cancer. Oncotarget. 2016;7:4994860.

249. Tsai KW, Lo YH, Liu H, Yeh CY, Chen YZ, Hsu CW, Chen WS, Wang JH. Linc00659, a long noncoding RNA, acts as novel oncogene in regulating cancer cell growth in colorectal cancer. Mol Cancer. 2018;17:72.

250. Zhang Y, Xiang C, Wang Y, Duan Y, Liu C, Jin Y, Zhang Y. IncRNA LINC00152 knockdown had effects to suppress biological activity of lung cancer via EGFR/PISK/AKT pathway. Biomed Pharmacother. 2017;94:644-51.

251. Cai Q, Wang ZQ, Wang SH, Li C, Zhu ZG, Quan ZW, Zhang WJ. Upregulation of long non-coding RNA LINC00152 by SP1 contributes to gallbladder cancer cell growth and tumor metastasis via PI3K/AKT pathway. Am J Transl Res. 2016;8:4068-81.

252. Gong J, Qi X, Zhang Y, Yu Y, Lin X, Li H, Hu Y. Long noncoding RNA linc00462 promotes hepatocellular carcinoma progression. Biomed Pharmacother. 2017;93:40-7.

253. Liu B, Pan S, Xiao Y, Liu Q, Xu J, Jia L. LINC01296/miR-26a/GALNT3 axis contributes to colorectal cancer progression by regulating O-glycosylated MUC1 via PI3K/AKT pathway. J Exp Clin Cancer Res. 2018;37:316.

254. Wu J, Cheng G, Zhang C, Zheng Y, Xu H, Yang H, Hua L. Long noncoding RNA LINC01296 is associated with poor prognosis in prostate cancer and promotes cancer-cell proliferation and metastasis. J Exp Clin Cancer Res. 2017;10:1843-52.
255. Min X, Liu K, Zhu H, Zhang J. Long noncoding RNA LINC003121 inhibits proliferation and invasion of thyroid Cancer cells by suppression of the Phosphatidylinositol-3-kinase (PI3K)/Akt signaling pathway. Med Sci Monit. 2018:24:4592-601.

256. Qin L, Jia Z, Xie D, Liu Z. Knockdown of long noncoding RNA urothelial carcinomaassociated 1 inhibits cell viability, migration, and invasion by regulating microRNA182 in gastric carcinoma. J Cell Biochem. 2018;119:10075-86.

257. Nasrollahzadeh-Khakiani M, Emadi-Baygi M, Nikpour P. Augmented expression levels of IncRNAs ecCEBPA and UCA1 in gastric cancer tissues and their clinical significance. Iran J Basic Med Sci. 2017;20:1149-58.

258. Lebda MA, Nasr NE, Nasr SM, El-Sayed Y, Li C, Liang G, Yang S, Sui J, Yao W, Shen $\mathrm{X}$, et al. Dysregulated IncRNA-UCA1 contributes to the progression of gastric cancer through regulation of the PI3K-Akt-mTOR signaling pathway. Environ Sci Pollut Res Int. 2017;8:93476-91.

259. Yang C, Li X, Wang Y, Zhao L, Chen W. Long non-coding RNA UCA1 regulated cell cycle distribution via CREB through PI3-K dependent pathway in bladder carcinoma cells. Gene. 2012;496:8-16.

260. Sadek KM. Role of IncRNAs as prognostic markers of hepatic cancer and potential therapeutic targeting by S-adenosylmethionine via inhibiting PI3K Akt signaling pathways. J Cell Biochem. 2018;25:20057-70.

261. Liu Z, Dou C, Yao B, Xu M, Ding L, Wang Y, Jia Y, Li Q, Zhang H, Tu K, et al. Ftx non coding RNA-derived miR-545 promotes cell proliferation by targeting RIG-I in hepatocellular carcinoma. Oncotarget. 2016;7:25350-65.

262. Goedert L, Pereira CG, Roszik J, Placa JR, Cardoso C, Chen G, Deng W, Yennu-Nanda VG, Silva WA Jr, Davies MA, Espreafico EM. RMEL3, a novel BRAFV600E-associated long noncoding RNA, is required for MAPK and PI3K signaling in melanoma. Oncotarget. 2016;7:36711-8.

263. Yu X, Yang Y, Li Y, Cao Y, Tang L, Chen F, Xia J. Baicalein inhibits cervical cancer progression via downregulating long noncoding RNA BDLNR and its downstream PI3K/Akt pathway. Int J Biochem Cell Biol. 2018;94:107-18.

264. Yu G, Liu G, Yuan D, Dai J, Cui Y, Tang X. Long non-coding RNA ANRIL is associated with a poor prognosis of osteosarcoma and promotes tumorigenesis via PI3K/Akt pathway. J Bone Oncol. 2018;11:51-5.

265. Dong X, Jin Z, Chen Y, Xu H, Ma C, Hong X, Li Y, Zhao G. Knockdown of long non-coding RNA ANRIL inhibits proliferation, migration, and invasion but promotes apoptosis of human glioma cells by upregulation of miR-34a. J Cell Biochem. 2018;119:2708-18.

266. Zhang D, Sun G, Zhang H, Tian J, Li Y. Long non-coding RNA ANRIL indicates a poor prognosis of cervical cancer and promotes carcinogenesis via PI3K/Akt pathways. J Cell Biochem. 2017:85:511-6.

267. Shi H, Pu J, Zhou XL, Ning YY, Bai C. Silencing long non-coding RNA ROR improves sensitivity of non-small-cell lung cancer to cisplatin resistance by inhibiting PI3K/Akt/mTOR signaling pathway. Tumour Biol. 2017;39: 1010428317697568.

268. Song W, Mei JZ, Zhang M. Long noncoding RNA PIncRNA-1 promotes colorectal Cancer cell progression by regulating the PI3K/Akt signaling pathway. Oncol Res. 2018;26:261-8.

269. Xu X, Yin Y, Tang J, Xie Y, Han Z, Zhang X, Liu Q, Qin X, Huang X, Sun B. Long non-coding RNA Myd88 promotes growth and metastasis in hepatocellular carcinoma via regulating Myd88 expression through H3K27 modification. Cell Death Dis. 2017;8:e3124.

270. Liu ML, Zhang Q, Yuan X, Jin L, Wang LL, Fang TT, Wang WB. Long noncoding RNA RP4 functions as a competing endogenous RNA through miR-7-5p sponge activity in colorectal cancer. World J Gastroenterol. 2018;24:1004-12.

271. Zhu M, Wang X, Gu Y, Wang F, Li L, Qiu X. MEG3 overexpression inhibits the tumorigenesis of breast cancer by downregulating miR-21 through the PI3KJAkt pathway. J Cell Biochem. 2018;661:22-30.

272. Xu DH, Chi GN, Zhao CH, Li DY. Long noncoding RNA MEG3 inhibits proliferation and migration but induces autophagy by regulation of Sirt7 and PIJK/AKT/mTOR pathway in glioma cells. J Cell Biochem. 2018. Epub ahead of print.

273. Deng R, Fan FY, Yi H, Liu F, He GC, Sun HP, Su Y. MEG3 affects the progression and chemoresistance of T-cell lymphoblastic lymphoma by suppressing epithelial-mesenchymal transition via the PI3K/mTOR pathway; 2018.

274. Zhang L, Liang X, Li Y. Long non-coding RNA MEG3 inhibits cell growth of gliomas by targeting miR-93 and inactivating PI3K/AKT pathway. Oncol Rep. 2017;38:2408-16.

275. Wang X, Wang Z, Wang J, Wang Y, Liu L, Xu X. LncRNA MEG3 has antiactivity effects of cervical cancer. Biomed Pharmacother. 2017;94:636-43.

276. Sun KX, Wu DD, Chen S, Zhao Y, Zong ZH. LncRNA MEG3 inhibit endometrial carcinoma tumorigenesis and progression through PI3K pathway. Apoptosis. 2017;22:1543-52. 
277. Gu L, Zhang J, Shi M, Zhan Q, Shen B, Peng C. IncRNA MEG3 had anticancer effects to suppress pancreatic cancer activity. Biomed Pharmacother. 2017;89:1269-76.

278. Shao Q, Xu J, Deng R, Wei W, Zhou B, Yue C, Zhu M, Huang X, Zhu H. Long non-coding RNA-422 acts as a tumor suppressor in colorectal cancer. Biochem Biophys Res Commun. 2018;495:539-45.

Ready to submit your research? Choose BMC and benefit from:

- fast, convenient online submission

- thorough peer review by experienced researchers in your field

- rapid publication on acceptance

- support for research data, including large and complex data types

- gold Open Access which fosters wider collaboration and increased citations

- maximum visibility for your research: over $100 \mathrm{M}$ website views per year

At $B M C$, research is always in progress.

Learn more biomedcentral.com/submissions 